\title{
44th Annual Meeting of the Cervical Spine Research Society
}

\author{
Toronto, Ontario, Canada • December 1 - 3, 2016 \\ (DOI: 10.3171/2017.2.FOC-CSRSabstracts)
}

\section{2nd Place Clinical Paper Award • \$1,000}

\begin{abstract}
Presentation \#1. Is Cervical Bracing Necessary after Single and Multi-Level Anterior Cervical Discectomy and Fusion? A Prospective Randomized Study
\end{abstract}

Samuel C. Overley, MD, New York, NY; Robert K. Merrill, BS, New York, NY; Evan O. Baird, MD, New York, NY; Samuel K. Cho, MD, Englewood Cliffs, NJ; Andrew C. Hecht, MD, New York, NY; Sheeraz A. Qureshi, MBA, MD, New York, NY

Study Design: Prospective randomized controlled trial.

Introduction: ACDF is on of the most common procedures performed on the cervical spine. Graft on union and subsidence are complications of the procedure. Rigid cervical collars restrict cervical motion post-operatively in an attempt to prevent such complications, but there is controversy regarding their effectiveness. The purpose of this study is to determine what effect, if any, cervical bracing after ACDF has on rates of subsidence, fusion, and patient reported outcomes.

Methods: The Cervical Spine Research Society Resident Fellow Grant funded this project. Thirty- three consecutive patients undergoing one or two level ACDF surgery were randomized into a group receiving no brace or group receiving a cervical brace for 6 weeks post-operatively. Neck Disability Index (NDI) scores were recorded preoperatively and at 24 months follow-up as a clinical outcome measure. Computed Tomography scans were read 1 year postoperatively to determine fusion rates, and subsidence was measured on follow up lateral cervical radiographs.

Results: Twenty-two patients were in the no-brace group and 22 patients in the brace group, with an average age of 49 and 54 , respectively. The no-brace group had a total of 31 operative levels, while the brace group had 33 operative levels. There was no statistically significant difference in post-op NDI scores between brace $(11.56 \pm 8.62)$ and no-brace $(7.28 \pm 7.54)$ group $(\mathrm{p}=0.1969)$, as shown in Figure 1. There was no difference in subsidence of all operative levels between the brace $(1.62 \mathrm{~mm} \pm 0.62)$ and no-brace $(1.44 \mathrm{~mm} \pm$ 0.88 ) group $(\mathrm{p}=0.5739)$, shown in figure 2 . Additionally, there was no difference in the fusion rates between the brace $(87 \%)$ and no-brace $(95 \%)$ group $(\mathrm{p}=0.5768)$

Conclusions: Our results suggest no advantage in wearing a cervical brace following one or two level ACDF surgery. There is a trend towards improved NDI scores, less subsidence, and increased fusion rates in patients who did not wear a cervical brace during the post-operative period, though these results lack statistical significance.

\section{1st Place Resident Fellow Paper Award • \$2,000}

Presentation \#2. The Effect of Local vs. Intravenous Steroids on Dysphagia and Dysphonia following Anterior Cervical Discectomy and Fusion (ACDF): A Single-Blinded, Prospective, Randomized Control Trial

Tyler J. Jenkins, MD, Chicago, IL; Reuben Nair, MD, Cleveland, $O H$;Brett D. Rosenthal, MD, Chicago, IL; Marco Mendoza, MD, Chicago, IL; Wellington K. Hsu, MD, Chicago, IL; Alpesh A. Patel, $M D$, River Forest, IL; Jason W. Savage, MD, Cleveland, $O H$

Study Design: Prospective randomized controlled study

Introduction: Dysphagia and dysphonia are the most common complications following anterior cervical discectomy and fusion (ACDF). Fortunately, most post-ACDF dysphagia is mild and transient, but in the limited number of patients that develop severe dysphagia it will have profound effects on overall health and surgical outcomes. Severe dysphagia places the patient at higher risk for dehydration, malnutrition, social isolation, aspiration, pneumonia, and death. Previous studies have demonstrated that intravenous (IV) and local steroids can decrease prevertebral soft-tissue swelling, however, no standardized studies have compared the efficacy of local steroid application to controls during ACDF on post-operative dysphagia and dysphonia. We conducted a prospective randomized clinical trial to assess the efficacy of intra-operative steroid administration (intravenous or local) on dysphagia and dysphonia after ACDF.

Methods: 72 patients undergoing ACDF for the treatment of cervical degenerative disease were recruited. Inclusion criteria were patients greater than 18 years undergoing ACDF for the treatment of radiculopathy or myelopathy. Exclusion criteria included: age under 18 years, operations for trauma / infection / tumor / revision, or general metabolic diseases (diabetes, heart disease, renal disease). Patients were randomized into three cohorts: control (no steroid), IV steroid (10 mg one-time intraoperative dose of IV dexamethasone), or local steroid groups ( $40 \mathrm{mg}$ of triamcinolone placed in the retropharyngeal space directly on the cervical plate). Subjects were blinded from which treatment arm they received. Primary outcomes were measured for dysphagia (Bazaz, Eat-10) and dysphonia (VHI-10) [6-8]. Secondary outcomes include Neck Disability Index (NDI) and Visual Analog Scale (VAS) for neck pain. Patient outcomes were collected preoperatively, post-operative day 1 , week 2 , and week 6 . Statistical analysis was completed with significance set at $\mathrm{p}<0.05$.

Results: Baseline patient reported outcomes for dysphagia, dysphonia, and neck pain were not significantly different between the groups. Day 1 post-operative patient outcomes scores showed a significant improvement in dysphonia (VHI-10 $\mathrm{p}=0.026)$ and neck pain $(p=0.025)$ in the local steroid group (Table 1, Figure 1). There was also a trend towards significant improvement of post-operative Day 1 dysphagia with the local steroid group $($ Bazaz $p=0.057)$. The local steroid cohort showed significant improvement in dysphagia $($ Bazaz $p=0.026$; Eat-10 $\mathrm{p}=0.011)$ and neck pain $(\mathrm{p}=0.042)$ at 2 weeks post-operative when compared to the other treatment groups. At 6 weeks post-operative the local group had significantly less severe dysphagia (Bazaz $p=0.001$; Eat-10 $p<0.001$ ) when compared to the other treatment groups.

Conclusion: Local steroid application at the conclusion of cervical plating in ACDF surgery yields better patient-reported outcomes for dysphagia, dysphonia, and neck pain, when compared to no steroid or IV steroid administration.

Presentation \#3. The Impact of Local Steroid Application on Dysphagia following an Anterior Cervical Discectomy and Fusion: Preliminary Results of a Prospectively, Randomized, Single-Blind Trial

Kern Singh, MD, Chicago, IL; Dustin H. Massel, BS, Northbrook, IL; Benjamin C. Mayo, BA, Chicago, IL; Junyoung Ahn, BS, Chicago, IL; Daniel D. Bohl, MD, MPH, Chicago, IL; Krishna D. Modi, BS, Schaumburg, IL; William W. Long, BA, Chicago, IL

Introduction: Intraoperative local steroid application has been theorized to reduce swelling and to improve swallowing in the immediate postoperative period following an anterior cervical discectomy and fusion (ACDF). As such, the purpose of this study is to quantify the impact of intraoperative local steroid application 
on patient-reported swallow function and postoperative swelling following an ACDF.

Materials and Methods: Total of 56 patients undergoing a 1or 2-level ACDF were randomized to depomedrol (DEPO) or no depomedrol (NODEPO) cohorts, receiving 1cc depomedrol or 1cc saline, respectively, applied to the surgical site using a gel-foam carrier (retroesophageal). The results of the SWAL-QOL questionnaire were compared between cohorts. Using pre- and postoperative lateral radiographs, a ratio of the prevertebral swelling distance to the anteriorposterior diameter of each vertebral body level was calculated for the index level (operative level), 2 vertebral levels above and below to obtain a swelling index. Similarly, the air index was calculated using the tracheal air window diameter. Any changes in these ratios (preoperative, 1-day, 6-weeks, and 12-weeks postoperatively) were compared between cohorts.

Results: Of the 56 patients, 32 patients $(57.1 \%)$ and 24 patients $(42.9 \%)$ were randomized to the DEPO and NODEPO groups, respectively. The DEPO cohort demonstrated a higher percentage of smokers $(15.6 \%$ vs. $0.0 \% ; \mathrm{p}=0.042)$. There were no differences in patient demographics, preoperative characteristics, or the mean change in scaled total SWAL-QOL score between the DEPO and NODEPO patients at any postoperative time point (6-weeks: $\mathrm{p}=0.505$; 12 weeks: $\mathrm{p}=0.487$ ). Lastly, the mean change in both swelling and air indices were no different between cohorts.

Conclusions: The preliminary results of this prospective, randomized, single blinded study do not demonstrate a significant impact of local intraoperative steroid application on patient-reported swallowing function or postoperative swelling following an ACDF. Both cohorts exhibit an increase in radiographic swelling in the immediate postoperative period, which subsides to near normal levels by 12 -weeks postoperatively. Administration of DEPO also did not lead to an earlier hospital discharge compared to the NODEPO cohort. Additionally, patient reported swallowing scores did not correlate with changes in radiographic swelling or airway diameter. Enrollment of additional patients is ongoing and will help determine the true impact of local intraoperative steroid application on patient- reported dysphagia.

\section{Presentation \#4. A Prospective Comparative Study in Skin Antiseptic Solutions for Posterior Spine Surgeries: Chlorhexidine- Gluconate Ethanol vs. Povidone-Iodine}

Toshitaka Yoshii, MD, PhD, Tokyo, Japan; Takashi Hirai, MD, PhD, Tokyo, Japan; Kenichiro Sakai, MD, PhD, Tokyo, Japan; Atsushi Okawa, MD, PhD Tokyo, Japan; Kenichi Shinomiya, MD, PhD, Tokyo, Japan

Introduction: Surgical site infection (SSI) in spinal surgeries is associated with increased morbidity, mortality, length of hospitalization, and medical costs. Since the patient's skin is a major source of pathogens that can cause SSI, use of effective antiseptic solutions before surgery is important in limiting wound contamination and in preventing SSI. Previous studies have shown that chlorhexidinegluconate $(\mathrm{CHG})$ is more effective for skin antisepsis than povidoneiodine (PD-I) in general surgeries and joint surgeries. However, to date, few studies have investigated the preoperative antiseptic solutions in spine surgery. Therefore, we conducted prospective comparative study to evaluate the efficacy of two standard antiseptic solutions, CHG and PD-I, in eliminating bacterial pathogens from the surgical site in posterior spine surgeries.

Methods: A total of 190 patients who received posterior spine surgeries were included in this study. Surgical skin preparation solutions were quasi-randomized based on the month when the surgery was performed: $0.5 \% \mathrm{CHG}$ with ethanol for 98 patients and $10 \%$ PV-I for 92 patients. Sterile culture swabs were used to obtain samples from skin adjacent to the planed incision site before preparation, after preparation, and after wound closure. Swab samples were evaluated for bacterial growth on sheep blood agar plates using a semi-quantitative technique. Unpaired t-test and Fisher's exact test were used for statistical analysis. Minimum sample size calculated in the power analysis for skin antisepsis was 168 under the condition of the effect size of $0.22, \alpha=0.05, \beta=0.8$.

Results: No difference was found in patients' age, gender, diseases, surgical site (cervical or thoracolumbar), operating time, and intraoperative blood loss between the $\mathrm{CHG}$ and PD-I group (Table 1). Prior to surgical skin preparation, bacteria grew on culture of specimens from $83.7 \%$ of the patients: no significant difference was found between the CHG (81.5\%) and PD-I group (85.7\%). The common organisms isolated from surgical sites were Staphylococcus sp., Corynebacterium sp. and Bacillus sp. both in cervical and lumbar spine. After the preparation, there were no significant differences in the culture positive rate between the CHG (3.1\%) and PD-I group $(5.4 \%)($ Table 2$)$. The culture positive rates were increased after wound closure (pre-ope 4.2, post-ope $8.4 \%: \mathrm{p}=0.07$ ), and the positive samples were more common in cervical spine $(15.6 \%)$ compared with lumbar spine $(6.4 \%)$. The positive rates after wound closure in the PD-I group (14.1\%) was higher compared with the CHG group $(5.1 \%)(\mathrm{p}=0.05)$. However, no difference was found in infection rates between the 2 groups (CHG:1.0\%, PD-I: $3.3 \%, p=0.29$ ).

Conclusions: While CHG ethanol and PD-I were equally effective to eliminate the bacterial flora from the surgical site, $\mathrm{CHG}$ ethanol showed more favorable long-lasting effect for skin antisepsis in posterior spine surgeries.

Presentation \#5. Prospective Study of Deep Vein Thrombosis in Patients Associated with Degenerative Cervical Spine Surgery

Katsuhisa Yamada, MD, Sapporo, Japan; Kota Suda, MD, Hokkaido, Japan; Satoko M. Harmon, MD, Hokkaido, Japan; Miki Komatsu, MD, Hokkaido, Japan; Chikara Ushiku, MD, Hokkaido, Japan; Masahiko Takahata, MD, Sapporo, Japan

Introduction: Deep vein thrombosis (DVT) is a potentially fatal complication because of the risk of pulmonary thromboembolism development. Prevention, early detection and timely treatment of DVT are very important during the perioperative period of spine surgery. It is reported that DVT has a high incidence in patients with spinal cord injury. However, there have been few reports on perioperative DVT in patients undergoing degenerative cervical spinal surgery. The purpose of this study is to elucidate the incidence and risk factors for DVT associated with degenerative cervical spinal surgery.

Materials and Methods: Between April 2008 and March 2015, 761 patients who underwent cervical spinal surgery in our hospital were enrolled in this study. The exclusion criteria were spinal cord injury, traumatic disease, infectious disease, inflammatory disease including rheumatoid arthritis, neoplastic disease and receiving anticoagulation medication. Leg vein ultrasonography was carried out performed preoperatively and 4 days after surgery. All patients received treatment with intermittent pneumatic compression and elastic stockings for primary DVT prophylaxis. No anticoagulation medications were used for DVT prophylaxis. Statistical analysis was performed using binomial logistic regression analysis

and Fisher exact probability test.

Results: A total of 289 patients with cervical degenerative disease undergoing cervical spinal surgery (203 males, 86 females; average age: $66.5 \mathrm{yr}$ ) met the inclusion criteria in this study. The overall incidence of DVT was $3.1 \%(9 / 289)$ in degenerative cervical spinal surgery. All 9 cases with positive DVT were women and had distal DVT without proximal DVT. There were no patients with clinical signs of DVT. The incidence of preoperative DVT was 1.1\% (3 / 284, excluding 5 cases of not examined). The incidence of postoperative DVT was $2.1 \%$ ( $6 / 286$, excluding 3 cases of preoperative positive DVT cases). Statistically significant risk factor for preoperative DVT included female sex $(\mathrm{P}=0.024)$ in the univariate analysis. The risk factors related with the surgery, including operation time, intraoperative blood loss and surgical approach, were not significantly 
associated with postoperative DVT. The univariate analysis showed that statistically significant risk factors for perioperative DVT included female sex $(\mathrm{P}<0.001)$, old age $(\mathrm{P}=0.04)$, low Japanese Orthopaedic Association score $(\mathrm{P}=0.03)$, rapidly progressive myelopathy $(\mathrm{P}=$ $0.001)$ and Frankel grade $\mathrm{A}-\mathrm{C}(\mathrm{P}=0.01)$. The multivariate analysis showed that rapidly progressive myelopathy $(\mathrm{P}=0.04)$ was risk factor.

Conclusion: The incidence of perioperative DVT in patients undergoing degenerative cervical spine surgery was 3.1\%. Female sex, advanced age, gait inability and rapidly progressive myelopathy could be at high risk of developing DVT during the perioperative period of cervical spine surgery. This result indicates that screening for DVT is needed in patients who are at high risk for DVT.

\section{Presentation \#7. Similar Outcomes of Hybrid TDR / ACDF at 5-Year Follow-up}

\section{Glenn Buttermann, MD, Stillwater, $M N$}

Introduction: Cervical total disc replacement, TDR, is increasingly being accepted by third party payers in the U.S. as a surgical treatment option for patients who have failed conservative care of single level conditions. However, coverage for multilevel disease, which is more common, is still restricted to fusion surgery. The purpose of this study was to compare off-label hybrid TDR / ACDF to multilevel ACDF for multilevel cervical disease.

Materials and Methods: Four cohorts of patients were evaluated pre- and postoperatively with self-assessment patient-based outcomes questionnaires (VAS pain for neck and arm, pain drawing, disability, and use of pain medication). All patients had a minimum of three years of follow-up. Multilevel TDR / ACDF hybrid cohort, $n=43$, were compared to multilevel ACDF cohort, $\mathrm{n}=90$. Control groups were included consisting of single level TDR and ACDF patients to validate the study against published single level TDR vs. ACDF studies. Secondary surgeries were also analyzed for all cohorts.

Results: There were no demographical differences between the two cohorts. Both hybrid TDR / ACDF and multilevel ACDF groups had similar preoperative pain and disability and both had significantly improved outcomes after surgery. There were no differences in outcomes for any of the measures between hybrid and multilevel ACDF patients. Pain medication usage was decreased; preoperatively in the hybrid group 50\% took narcotics and $81 \%$ NSAIDs. Two years postop, $13 \%$ used narcotics and $45 \%$ used NSAIDs. Secondary surgeries were similar for the multilevel cohorts. At 5 years postop, adjacent level surgery was $9 \%$ in both the hybrid and the multilevel ACDF cohort. Pseudarthrosis repair was $9 \%$ vs. $11 \%$ in the hybrid and multilevel ACDF cohorts respectively. Although implant costs were less for the single level TDR patients compared to single level ACDF; for the multilevel case, costs were greater for the hybrid relative to the multilevel ACDF group. The study appears valid in that our single level TDR vs ACDF results were similar to that reported in multiple prior studies and found slightly better outcomes for single level cervical TDR relative to ACDF.

Conclusions: Hybrid TDR /ACDF, used off-label, gives comparable outcomes to multilevel ACDF patients at short term follow-up. Secondary surgeries due to pseudarthroses and adjacent segment disease was similar in both multilevel cohorts at the 5-year follow-up period. A hybrid procedure is a viable treatment option for patients with multilevel cervical disease.
Presentation \#8. Prestige Cervical Disc Arthroplasty vs. Anterior Cervical Discectomy / Fusion: 84 Month IDE Outcomes of Two Level, Prospective, Randomized Clinical Trial

Scott D. Hodges, DO, Chattanooga, TN; Matthew F. Gornet, MD, Chesterfield, MO; Todd H. Lanman, MD, Beverly Hills, CA; J. Kenneth Burkus, MD, Columbus, GA; Randall F. Dryer, MD, Austin, TX; Jeffrey R. McConnell, MD, Allentown, PA

Introduction: Cervical disc degeneration remains one of the most common spinal disorders requiring treatment. Patients failing conservative care have frequently been treated with anterior cervical discectomy and fusion (ACDF). Concern regarding rapid development of adjacent segment degeneration (ASD) has lead to the rapid development of motion sparing cervical disc arthroplasty prosthesis. Cervical disc degeneration requiring surgical management is contiguous at two levels in up to $40 \%$ of patients. Meta analysis of single level CDA vs. ACDF have shown superiority with lower rates of adjacent segment degeneration and fewer adjacent segment reoperations in the CDA group. Five year follow up of two level Cervical Disc Arthroplasty (CDA) vs. ACDF IDE study show similar findings as one level. There is greater probability of success for adjacent segment degeneration and lower reoperation at adjacent segments in the CDA group. The current study evaluates the outcomes and results in efficacy and safety of two level contiguous cervical arthroplasty vs. ACDF in a level 1 IDE control, randomized, prospective trial. CDA group has superiority in results compared to anterior cervical discectomy and fusion (ACDF).

Materials and Methods: A FDA approved level one clinical trial was carried out at 30 centers in the US comparing the radiographic and clinical results of a low profile ball and trough titanium-ceramic prosthesis vs. ACDF in 397 total patients with C3-7 two level radiculopathy and or myelopathy. All patients have failed at least 6 weeks conservative care and were randomized to investigational CDA

(209) vs. Control ACDF (188). Patient follow up was 7 years (84 months). Bayesian statistical analysis was used to evaluate primary endpoints of overall success as well as safety and efficacy endpoints. The overall success is a composite of the following criteria: 1.) neck disability index (NDI) improvement of $\geq 15$ points, 2.) maintenance or improvement in neurologic status, 3.) No serious adverse events caused by the implant or both the implant and the surgical procedure, no additional surgery (non elective implant removal, revision surgery, or supplemental fixation). Other endpoints included neck and arm pain numeric rating, work status, patient satisfaction, disc height, SF-36, and adverse events (AE).

Results: Clinical and radiographic follow up was $73.7 \%$ for the investigational CDA group and $67 \%$ for the control ACDF group. In the overall success category the CDA outcomes were statistically superior to ACDF (observed rate of $78.6 \%$ vs. 62.7\%; Posterior Probability of Superiority (PPS) $=99.8 \%$ ). The neurological success observed rate $(91.6 \%$ vs. $82.1 \%$; PPS $=99.0 \%)$ and the NDI success observed rate $(87.0 \%$ vs. $75.6 \%$; PPS $=99.2 \%)$ both were superior for the CDA vs. ACDF. Both groups have statistical improvement from base line for both neck and arm pain scores, neurologic success, SF-36 scores, and NDI. CDA and ACDF groups had no statistical differences in overall implant / surgical procedure related adverse events $(26.6 \%$, $27.7 \%$ respectively). When considering grade 3-4 adverse events, CDA group had statistically fewer (3.2\% vs. 7.2\%, Log Hazard Ratio (LHR) 95\%, Bayesian Credible Interval (BCI): -1.19( -2.29-0.15)) secondary surgical procedures at the index level in the CDA group were less in the CDA group vs. ACDF $(4.2 \%$ vs. $14.7 \%$, LHR = $95 \%$, BCI: $-1.29(-2.12,-0.46))$. The CDA group vs. ACDF group had fewer adjacent segment surgeries (6.5\% vs. $12.5 \%)$. The CDA group maintained angular motions of $6.5^{\circ}$ superiorly and $6.3^{\circ}$ inferiorly. Heterotopic ossification grade 4 occurred in $8.6 \%$ of superior level and $7.3 \%$ of inferior level patients.

Conclusion: Seven year outcomes of this low profile cervical disc arthroplasty compared to ACDF for cervical degenerative disc disease at two continuous levels had overall success superiority and shows 
CDA to be a safe and effective treatment while allowing maintenance of motion at both levels.

\section{Presentation \#9. Elevated Risk for Repeated Surgery after ADR Compared to ACDF in a Cohort of 715 Patients - A Retrospective Study with Minimum Five-Year Follow-up}

\section{Martin Skeppholm, MD, PhD, Lowenstromska Sjukuset, Sweden}

Introduction: Rates of repeated surgery after artificial disc replacement (ADR), has in several comparative studies shown to be lower than after fusion (ACDF) surgery in the cervical spine. The main causes for reoperation after ACDF has shown to be adjacent segment disease (ASD) and pseudarthrosis. One of the aims with ADR is to maintain motion and thus prevent ASD, which could be a long-term side effect of ACDF. Long-term side effects from ADR surgery as wear, instability, migration and heterotopic ossification also must be considered. The purpose of this retrospective study was to compare the incidence of repeated surgery between the different surgical techniques in a cohort with long-term follow-up.

Material and Methods: 715 patients treated with ACDF, ADR or a posterior procedure (PP) because of cervical radiculopathy or myelopathy over a time period of ten years $(2000-2010)$, were followed regarding rates of repeated surgery. Repeated surgery was defined as any secondary cervical surgery. Cause for repeated surgery as well as choice of new intervention was evaluated. Data was collected from a single-center setting.

Results: $79(11 \%)$ patients underwent a new operation during follow-up, which was minimum five years. Average time between primary and secondary intervention was 35 months. 50 / 504 (10\%), $27 / 172(15 \%)$ and $2 / 39(5 \%)$ were registered with a secondary intervention in the ACDF group, ADR group and PP group respectively. There was a statistically significant higher risk of repeated surgery in the ADR group compared to the ACDF group, OR 1.7 (C.I. $1.06-$ $2.8), p=0.03$. Risk for repeated surgery at index level was even higher for ADR, OR 5.1 (C.I. $2.4-10.7$ ), and $\mathrm{p}<0.001$. Among the patients who underwent a second intervention, operation because of adjacent segment disease (ASD) were more likely in the ACDF group, OR 3.1 (C.I. $1.1-8.6), p=0.03$. Reoperation rate because of ASD in the whole cohort did not differ between ACDF and ADR groups, $p=0.40$. Nor could any statistically significant difference be seen in comparison between the PP group and the ADR or the ACDF group regarding reoperation for ASD.

Conclusion: Artificial disc replacement showed higher risk for repeated surgery in this cohort and was not protective against secondary intervention because of adjacent segment pathology. It was more common with repeated surgery at the index level after disc replacement, indicating more implant related problems.

\section{Presentation \#10. Artificial Disc Replacements do Not Prevent Adjacent Segment Degeneration in the Cervical Spine}

Anna M. MacDowall, MD, Uppsala, Sweden; Nuno Canto Moreia, MD, PhD, Uppsala, Sweden; Martin Skeppholm, MD, PhD, Stockholm, Sweden; Catharina Marques, MD, Uppsala, Sweden; Yohan Robinson, MD, Uppsala, Sweden; Claes Olerud, MD, PhD, Uppsala, Sweden

Introduction: Adjacent segment degeneration (ASD) is degenerative changes in the level immediately adjacent to a fused level. It has been described to be a consequence of increased stress in the adjacent disc as a side effect of cervical immobilization. Artificial disc replacements (ADR) have been developed to preserve motion in the index level and subsequently prevent development of ASD. There are no sufficient data that they actually succeed with that and the question remains if ASD is caused by the influence of the fusion or by intrinsic disc aging processes. The aims of this study were to compare two surgical treatment methods for cervical radiculopathy, fusion and $\mathrm{ADR}$, regarding development of symptomatic and radiologic ASD as well as reoperations for ASD, at a five-year follow-up.

Methods: A prospective randomized controlled study with 151 patients undergoing surgery for cervical radiculopathy due to degenerative disc disease (DDD) was performed. The patients were randomized to either anterior decompression and fusion (ACDF) or anterior decompression and insertion of an ADR. There were 73 men and 78 women with the mean age of 46.85 years. MRI and neurologic examination was done preoperatively and at five years. Reoperations for ASD were also accounted for. A neuroradiologist examined all the MRI's according to a five level grading system describing the nucleus signal intensity, the nucleus structure, distinction of nucleus and annulus and the disc height. Symptomatic ASD was defined as radiculopathy confirmed with a neurologic examination and consistent with degenerative changes on MRI at the five-year follow-up. Fisher's exact test was used for comparison between groups according to symptomatic ASD and a two-sample t-test compared the radiologic ASD measured as delta-MRI grade in the two groups.

Results: 26 patients were lost to follow-up and in 13 patients the quality of the MRI was not sufficient for the grading assessment, remaining 112 patients to analyze. There were 10 patients, 3 females and 7 males, with symptomatic ASD in the ACDF group compared to 16 patients, 10 females and 6 males, in the ADR group. The difference was not statistically significant, $\mathrm{p}=0.66$. The mean increase in degeneration grade between preoperative MRI and five years MRI was 0.67 in the ACDF group and 0.39 in the ADR group. That was statistically significant with a p-value of 0.015 . Five patients in the ACDF group and five patients in the ADR group had been reoperated for ASD within the five year follow up.

Conclusions: There are no differences in the development of symptomatic ASD five years after surgery with either ACDF or ADR. An increase of radiologic ASD seen on MRI, disregarding if the patients have symptoms or not, is more frequent in the ACDF group. The same amount of patients in the ACDF- and ADR group has so severe ASD that they have to go through with a second surgery within five years.

\section{Presentation \#11. Tobacco Smoking and Outcomes of Decompressive Surgery in Patients with Symptomatic Degenerative Cervical Myelopathy}

Paul M. Arnold, MD, FACS, Kansas City, KS; Branko Kopjar, MD, PhD, Seattle, WA; Lindsay A. Tetreault, PhD, Toronto, ON, Canada; Hiroaki Nakashima, MD, PhD, Nagoya, Japan; Michael G. Fehlings, MD, PhD, Toronto, ON, Canada

Introduction: Tobacco smoking has been associated with poor outcomes following surgery for cervical radiculopathy. However, the impact of tobacco smoking on outcomes following surgery for degenerative cervical spondylotic myelopathy (DCSM) has not been extensively evaluated.

Materials and Methods: This study analyzed prospectivelycollected data from two large multicenter international cohort studies. Outcome measures were preoperative smoking status, modified Japanese Orthopedic Association scale (mJOA), Nurick score, Neck Disability Index (NDI), Short Form 36v2 (SF- 36v2 ${ }^{\mathrm{TM}}$ ), and the 30 -meter walk test (30MWT). Analysis of Covariance was used to evaluate differences in outcomes at 12 months between smokers and nonsmokers while controlling for relevant baseline characteristics.

Results: 749 patients with symptomatic DCSM underwent surgical decompression at 24 international sites. There were 547 $(73 \%)$ nonsmokers and $202(27 \%)$ smokers. After imputation of missing 12- month scores, $694(92.66 \%)$ subjects had 12-month data. Smokers were younger (average 53.40 vs 57.42 years) and had 
worse preoperative NDI, SF-36v2 Physical Component Score (PCS) and SF-36v2 Mental Component Score (MCS) $(\mathrm{p}<0.1)$. There were no differences in gender, race, symptom duration, etiology, number of operated levels, disease severity, or complication or reoperation rates. At 12 months, improvements in mJOA, NDI, and SF-36v2 PCS outcomes were $15.59 \%, 31.61 \%$, and $28.57 \%$ lower in smokers compared to nonsmokers. Following adjustment for confounders, these differences remained significant.

Conclusions: Preoperative tobacco smoking is strongly associated with suboptimal clinical, functional, and quality of life outcomes in patients undergoing surgery for DCSM. While both nonsmokers and smokers benefited from surgical decompression, the extent of improvement was higher in nonsmokers than smokers.

\section{Presentation \#12. Comparative Effectiveness between Laminectomy with Fusion and Laminoplasty for the Treatment of Multilevel Cervical Spondylotic Myelopathy}

Colin M. Haines, MD, Cleveland, OH; Heath Gould, Cleveland, $\mathrm{OH}$; Emily Hu, Cleveland, OH; Jacob A. Miller, Cleveland, OH; Roy Xiao, R, Cleveland, OH; Thomas E. Mroz, Cleveland, OH; Don K. Moore, $\mathrm{MD}$, Cleveland, $\mathrm{OH}$

Introduction: The optimal management of multilevel cervical spondylotic myelopathy (CSM) remains unknown. Both laminectomy with fusion and laminoplasty have been demonstrated to offer a clinical benefit in small retrospective investigations. However, given the cost of spinal fusion, laminoplasty may represent a more costeffective alternative that is equally efficacious.

Methods: A retrospective cohort study was conducted among patients undergoing cervical decompression for the treatment of multilevel CSM. The EQ-5D, PDQ, and PHQ-9 instruments were prospectively-collected between 2008 and 2015. These instruments served as measures of overall quality of life (QOL), pain-related disability, and depression. Postoperative QOL improvement exceeding the EQ-5D minimum clinically important difference (MCID) was the primary outcome. Secondary outcomes included the total surgical episode of care cost, PDQ MCID, and PHQ-9 MCID. The surgical episode of care was defined in three periods: 30 days prior to admission, the index admission, and discharge to 365 days after admission. To present costs from the payer's perspective, costs were normalized to national Medicare reimbursement and presented in 2014 USD. Unpaired continuous and categorical data were compared via Wilcoxon rank-sum and Fisher's exact tests, while paired data were compared with Wilcoxon signed-rank tests. Multivariable logistic and log-transformed linear regression were used to model EQ-5D MCID and total episode of care costs.

Results: 186 patients were eligible for inclusion; among these, 142 (76\%) underwent laminectomy with fusion, while 44 (24\%) underwent laminoplasty. No significant differences in demographic or comorbid characteristics were observed. Preoperatively, the mean EQ-5D index was marginally greater in the laminoplasty cohort $(0.530$ vs. $0.581, p$ $=0.17$. Similarly, mean EQ-5D perceived health (41 vs. $50, \mathrm{p}=0.06)$, PHQ-9 (4.7 vs. 2.9, $\mathrm{p}=0.20$ ), and total PDQ (41 vs. $31, \mathrm{p}=0.47$ ) demonstrated poorer preoperative QOL in the laminectomy cohort. Within 30 days prior to admission, median disease-specific costs were not significantly different between cohorts ( $\$ 818$ vs. $\$ 716, p=0.21$ ). However, median costs for the index admission were significantly greater among patients undergoing laminectomy ( $\$ 25,888$ vs.

$\$ 19,427, \mathrm{p}<0.001)$. Postoperatively, mean EQ-5D index improved to $0.592(\mathrm{p}=0.02)$ and $0.664(\mathrm{p}=0.01)$ in the laminectomy and laminoplasty cohorts, respectively. The proportion of patients achieving an EQ-5D MCID did not significantly differ between cohorts $(36 \%$ vs. $30 \%, \mathrm{p}=0.47)$. Following multivariable logistic regression, surgical type was not significantly associated with EQ-5D MCID (laminectomy vs. laminoplasty: OR $0.86, p=0.70$ ). In the period following discharge, costs were not significantly different between cohorts $(\$ 3,450$ vs. $\$ 3,424, p=0.46$ ). However, total episode of care costs were greater in the laminectomy with fusion cohort $(\$ 34,718$ vs. $\$ 25,260, p<0.001)$. Following multivariable linear regression, total episode of care costs remained significantly greater in the laminectomy cohort $(\beta=0.572, \mathrm{p}<0.001)$, corresponding to a mean difference of $\$ 16,392$.

Conclusions: Poorer preoperative QOL was observed among patients undergoing laminectomy with fusion relative to laminoplasty, and therefore these populations may differ with respect to expected surgical benefit. However, the proportion of patients achieving a clinically-relevant QOL improvement did not significantly differ, suggesting similar efficacy. After controlling for differences in baseline characteristics, laminoplasty appeared to be more costeffective relative to laminectomy with fusion.

Presentation \#13. Comparisons of Anterior and Posterior Surgery for Cervical Spondylotic Myelopathy - A Propensity Score Matched Analysis using AOSpine CSM North America and International Database

So Kato, MD, Tokyo, Japan; Aria Nouri, MD, MSc, Richmond, ON, Canada; Dongjin Wu, Ji-Nan, China; Satoshi Nori, MD, PhD, Chiba, Japan; Lindsay A. Tetreault, BS, PhD, Oakville, ON, Canada ; ichael G. Fehlings, MD, PhD, Toronto, ON, Canada

Introduction: Both anterior and posterior approaches are established as decompression for cervical spondylotic myelopathy (CSM) with similarly optimal neurological recovery. They are often chosen by surgeons' preferences based on the factors including the patients' history, radiographic features of spinal cord compression and alignment. However, each surgical approach has its pros and cons in terms of complication rates, spinal alignment and there is lack of consensus on which approach is preferable. Comparative studies are limited with mixed conclusions due to selection biases (Fehlings, Spine 2013). Designing a randomized controlled trial for surgical decision making is challenging due to ethical constraints. The objective of the present study is to compare the postoperative outcome between anterior and posterior decompression surgery for CSM by the propensity score matched analysis using the multicenter prospective database.

Materials and Methods: AOSpine CSM North America and International studies are prospective, multicenter databases for surgical CSM patients, which enrolled 278 and 479 consecutive cases in 12 and 16 sites, respectively. $59.0 \%$ of patients were treated anteriorly and $37.9 \%$ were treated posteriorly. A minority (3.0\%) underwent a 2-stage anteroposterior surgery and was excluded from the analysis. Patient demographic data, pre- and 2-year post-operative radiographic images (MRI and x-ray), surgical details, pre- and post-operative neurological status and complication data were reviewed. Among them, age, sex, body mass index, the spectrum of degenerative changes that contributed to the spinal cord compression investigated in preoperative MRI (single-level disc pathology, multi-level disc pathology, enlargement of PLL, enlargement of LF, subluxation / listhesis, congenital fusion and number of compression levels), C2-7 Cobb angle on pre-operative $\mathrm{x}$-ray and pre-operative mJOA score were used in multiple logistic regression analysis to determine the propensity score for anterior approach for decompression. One-to-one matching was performed to adjust for patients' background characteristics, and neurological recovery and spinal alignment and complication rates were compared between anterior and posterior group.

Results: 260 cases were included in propensity score calculation, and one-to-one matching resulted in 40 pairs of anterior and posterior surgery. Operation time (173 vs. 173 mins, $p=0.76)$, length of stay (12.0 vs. 9.3 days, $\mathrm{p}=0.57$ ), post-operative mJOA (15.0 vs. $14.9, \mathrm{p}$ $=0.67)$, recovery rate of mJOA $(46.5 \%$ vs. $47.3 \%, p=0.85)$, SF-36 PCS (41.6 vs. $41.2, \mathrm{p}=0.85)$ and NDI (21.8 vs. $22.1, \mathrm{p}=0.75)$ were not statistically significantly different between anterior and posterior 
surgery. The differences in postoperative C2-7 Cobb angle $\left(15.5^{\circ}\right.$ vs. $\left.10.4^{\circ}, \mathrm{p}=0.14\right)$, T1 slope - cervical lordosis mismatch $\left(34.7^{\circ} \mathrm{vs}\right.$. $\left.28.2^{\circ}, \mathrm{p}=0.09\right)$ and $\mathrm{C} 2-7$ sagittal vertical axis $(26.5$ vs. $26.6 \mathrm{~mm}$, $\mathrm{p}=0.45$ ) did not reach statistical significance. Dysphagia was only reported in anterior group $(2.5 \%$ vs. $0 \%)$, and C5 palsy was only reported in posterior group ( $2.5 \%$ vs. $0 \%)$. Perioperative complications were equally reported in both groups (10\% vs. $10 \%)$.

Conclusion: Anterior and posterior decompression for CSM showed similar post-operative neurological recovery and outcomes, although the spectrums of complications were different.

Presentation \#14. Postoperative Walking Ability of NonAmbulatory Cervical Myelopathy Patients Yoshiki Takeoka, MD, Kobe, Japan

Shuichi Kaneyama, MD, PhD, Kobe, Japan; Masatoshi Sumi, MD, PhD, Kobe, Japan; Koichi Kasahara, MD, PhD, Kobe, Japan; Aritetsu Kanemura, MD, PhD, Kobe, Japan; Masato Takabatake, Kobe, Japan; Akihiro Koh, MD, Kobe, Japan; Hiroaki Hirata, MD, PhD, Kobe, Japan; Masanori Tsubosaka, Kobe, Japan

Introduction: Many researchers have reported the outcome after surgical treatment in cervical myelopathy, however, regarding to severe gait disturbance, what extent of improvement could be obtainable has not been clarified yet. We investigated the postoperative improvement of the ambulatory level and prognostic factors in non-ambulatory patients with cervical myelopathy.

Materials and Methods: A total of 131 non-ambulatory patients surgically treated due to cervical myelopathy ( 78 males, 53 females; mean age 71.5 years) were followed for an average of 3.0 years (range $1.0-8.6$ years). Their walking ability at the follow-up period was compared to the preoperative condition by Japanese Orthopaedic Association (JOA) scores and lower-extremity function subscores (L / E subscores); graded "excellent" (2 points or more), "good" (1.5 points), "fair" (one point), and "poor" ( 0.5 or 0 points). Disease durations (from the onset of myelopathy symptoms or gait disturbance to the time of surgery) were also investigated. The data were analyzed by the Wilcoxon signed-rank test and the chi-squared analysis $(\mathrm{p}<$ $0.05)$.

Results: Preoperative L / E subscore was one point in 71 patients, 0.5 in 30 patients, and 0 in 30 patients. The mean L / E subscore improved significantly from 0.7 to 1.6 points $(p<0.01)$. Fifty patients were graded as "excellent" (38\%) and 21 patients as "good" (16\%), indicating $54 \%$ of the improvement of non-ambulatory condition as to walk without support at the follow-up period. Seventy one patients whose preoperative L / E subscores presented one point improved significantly better than the other 60 patients (preoperative L / E subscores were less than one point), where the mean L / E subscore was 1.7 and 1.4 points respectively $(\mathrm{p}<0.05)$. On the assessment of the 60 non-ambulatory patients even with any support (preoperative L / E subscore; 0.5 or 0 points), 26 patients (43\%) recovered enough to walk without support and 17 patients $(28 \%)$ were graded as "excellent". Of those 60 patients, 17 patients graded as "excellent" had shorter durations of myelopathy symptoms and / or gait disturbance (7.9 and 3.8 months respectively) than the others (29.5 and 8.9 months respectively) $(\mathrm{p}<0.05)$. In these 60 patients, ROC curve showed the cut-off values of the duration of myelopathy symptoms and gait disturbance provided the improvement to "excellent" were three and two months. Twelve of 22 patients operated within three months from the onset of myelopathy were evaluated as "excellent", which was significantly high compared to five of 38 patients operated after three months $(p<0.01)$. Likewise, the onset of gait disturbance influenced their recoveries significantly in the patients operated within two months (12 of 27 patients) compared to after two months (5 of 33 patients $)(\mathrm{p}<0.01)$.

Conclusion: We demonstrated that $54 \%$ of non-ambulatory patients due to cervical myelopathy recovered up to the level without need for a support after surgery. To expect better walking ability, surgery should be performed while walking ability is reserved. Also, we concluded surgical treatment should be performed within three months after the onset of myelopathy or two months after the onset of gait disturbance for obtaining improvement from non-ambulatory condition with imperative support to stable gait (L/E subscore; 2 points or more).

\section{Presentation \#15. Impact of Preoperative Cervical Sagittal Balance on Surgical Treatment for Cervical Spondylotic Myelopathy Caused by Ossification of the Posterior Longitudinal Ligament}

Kenichiro Sakai, MD, PhD, Saitama, Japan; Toshitaka Yoshii, MD, PhD, Tokyo, Japan; Takashi Hirai, MD, PhD, Tokyo, Japan; Yoshiyasu Arai, Saitama, Japan; Yu Matsukura, MD, PhD, Saitama, Japan; Astushi Okawa, MD, PhD, Tokyo, Japan

Introduction: Recently, cervical sagittal balance has received increased attention as an important determinant of radiological and clinical outcomes after surgery. However, little is known about the precise impact of cervical sagittal balance on surgical outcomes, especially for patients with cervical myelopathy caused by ossification of the posterior longitudinal ligament (OPLL). We retrospectively investigated the surgical outcomes of cervical myelopathy caused by OPLL with special attention to the concept of the cervical sagittal balance.

Materials and Methods: The study included a total of 97 consecutive patients (78 male, 19 female; mean age 64.3 years) who underwent surgery for cervical myelopathy caused by OPLL at our hospital from 2008 and completed at least 1-year of follow-up. The average follow-up period was 3.1 years. We selected surgical procedures as follows: (1) For patients with massive OPLL or preoperative kyphotic cervical alignment, we performed anterior decompression and fusion with floating method (ADF) as the 1st choice, and posterior decompression and fusion (PDF) as the 2nd choice. (2) For patients with slight OPLL and normal cervical alignment, we performed laminoplasty (LAMP). ADF was performed in 39 cases, PDF in 18 cases and LAMP in 40 cases. Cervical lateral $x$-ray images taken in the neutral standing position were evaluated preoperatively and at the final follow-up visit. Radiographic measurements included the following: (1) CSVA, which was measured as the distance between a plumb line dropped from the anterior margin of the external auditory canal and the posterior-cranial corner of the C7 vertebral body, (2) CL (C2-7 lordotic angle) and (3) C7 slope. Clinical results were evaluated using the Japanese Orthopedic Association scoring system for cervical myelopathy (C-JOA score). We divided patients into two groups based on the preoperative CSVA: the Balance $(\mathrm{CSVA}<40 \mathrm{~mm})$ and Imbalance (CSVA $\geq 40 \mathrm{~mm}$ ) groups.

Results: Sixty-nine patients were in the Balance group, and 28 patients were in the Imbalance group. In the Balance group, none of the three operations had an effect on the CL. In contrast, in the Imbalance group, while ADF and PDF had no effect on the CL, LAMP worsened the CL postoperatively (Figure 1). None of three operations had an effect on the $\mathrm{C} 7$ slope in either group. The recovery rates of the C-JOA scores in the Balance group showed no significant differences among the three operations; however in the Imbalance group, LAMP resulted in worse recovery rate of the C-JOA score than ADF or PDF (Figure 2). In 7 cases where LAMP was performed in the Imbalance group, postoperative cervical kyphosis was observed in 4 cases (57.1\%), and recurrence of myelopathy was observed in 3 cases $(42.9 \%)$.

Conclusion: LAMP is not suitable for patients with cervical myelopathy caused by OPLL who have cervical sagittal imbalance, even in cases with normal preoperative alignment and slight OPLL. 


\section{2nd Place Resident Fellow Paper Award • \$1000}

Presentation \#16. Health Economic and Patient-Centered Analysis on the Value of Surgery for Degenerative Cervical Myelopathy: Strong Support for Surgical Intervention

Christopher D. Witiw, MD, Toronto, ON, Canada; Lindsay A. Tetreault, PhD, Toronto, ON, Canada; Fabrice Smieliauskas, PhD, Chicago, IL; Branko Kopjar, MD, PhD, Seattle, WA; Eric M. Massicotte, MD, MSc, Toronto, ON, Canada; Michael G. Fehlings, $M D, P h D$, Toronto, ON, Canada

Introduction: Degenerative Cervical Myelopathy (DCM) is the most common cause of non-traumatic spinal cord impairment in adults. Surgery has been shown to improve neurological symptoms and functional status, but it is costly. As healthcare sustainability concerns rise, the value of care has come to the forefront of policy decision-making. Evidence for both health related quality of life outcomes and costs are needed to inform medical policies. The aim of this analysis is to determine if surgery for DCM is cost effective and to provide an estimate of the lifetime incremental cost utility of the intervention.

Methods: All patients undergoing surgery for DCM at a Canadian tertiary care center between 2005 and 2011, who were enrolled in either the AOSpine CSM-North America or CSM-International studies were included. Health utility was measured at baseline and then 6,12 and 24-months following surgery using the Short Form-6D (SF-6D) health utility score. Costs were calculated on a patient level, from the hospital budgetary expenditures over the 24-month follow-up period. All costs were obtained from a micro-cost database and reported in Canadian dollars; inflated to January 2015 values. Quality adjusted life year (QALY) gain was estimated as an area under the curve with a linear interpolation. Lifetime incremental cost utility ratios (ICUR) for surgery were estimated using a Markov state transition model (Figure 1). Sensitivity to structural uncertainly arising from lifetime extrapolation and the single arm cohort design of the study was assessed by constructing supplementary constrained models. Deterministic and probabilistic sensitivity analyses were used to account for parameter uncertainty. All QALY gains and costs were discounted at $3 \%$ per annum.

Results: The analysis included 171 patients; follow-up at 2-years was $96.5 \%$. Mean age was $58.2 \pm 12.0$ years and baseline health utility was $0.56 \pm 0.14$. Average QALY gained over the 24-month following surgery was $0.14(95 \% \mathrm{CI}: 0.11-0.17, \mathrm{p}<0.001)$. The average 2 -year cost of treatment was

$\$ 19,218 \pm 12,404$. Cost associated with the surgery accounted for two-thirds $(65.7 \%)$ of the total costs. The remainder of the costs were for pre-surgical preparation, post-surgical recovery and re-operations. Three patients required a re-operation over the 2-year follow-up period, and accounted for $1.85 \%$ of the total costs. The estimated lifetime ICUR of surgical intervention was \$11,496.02 / QALY gained (Figure $2 \mathrm{~A}$ ), with $97.9 \%$ of model estimates (Figure $2 \mathrm{~B}$ and $2 \mathrm{C}$ ) meeting the criteria to be considered 'very cost effective' (\$54,000 CAD). Model structure sensitivity assessments revealed ICUR estimates that remained within the 'very cost effective' threshold, suggesting the findings are robust to the estimations of the lifetime model.

Conclusion: Our study suggests that surgery for DCM is associated with significant and clinically meaningful improvements in quality of life and is cost effective.
Presentation \#17. Resource Utilization for Anterior Compared to Posterior Surgical Approaches for Cervical Spondylotic Myelopathy: An Analysis of Private Payer and Medicare Databases

Sohrab S. Virk, MD, MBA, Columbus, OH; Frank M. Phillips, MD, Chicago, IL; Safdar N. Khan, MD, Columbus, $\mathrm{OH}$

Introduction: Cervical spondylotic myelopathy (CSM) is a progressive spinal condition that often necessitates surgery. Studies have shown the clinical equivalency of anterior vs. posterior approaches for CSM surgery. There has been no study comparing reimbursement from private third party payers and Medicare for anterior vs. posterior approaches for CSM surgery. This is important especially in the era of value-based clinical decision making. The purpose of this study is to determine the amount and type of resources used for anterior compared to posterior surgical treatment of CSM.

Methods: A retrospective review of two national claims databases was performed. This study comprised of two large cohorts of patients that underwent either an anterior or posterior approach for treatment of CSM. These patients were selected from a database of patients with Medicare and another database of patients with private payer health insurance.

The outcome measures were cost of a 90 day episode of care as well as a breakdown of cost components for each surgical procedure. A private payer database (HORTHO) and Medicare database (SAF5) were used to evaluate clinical and financial information between 2005 and 2014 for patients undergoing surgery for CSM. Reimbursement information was collected from the day of surgery to 90 days after the index procedure.

Results: A total of 16,444 patients were included within this analysis. Within the HORTHO database there were 10,332 and 1,556 patients treated with an anterior or posterior approach for CSM respectively. Within the SAF5 database there were 3,851 and 705 patients treated by an anterior or posterior approach for CSM respectively. The average reimbursement for anterior vs. posterior approaches within the HORTHO database was $\$ 20,863(+/-2,014)$ and $\$ 23,813(+/-\$ 4,258)$ respectively $(p=0.048)$. The average reimbursement for anterior vs. posterior approaches within the SAF5 database was \$18,219 ( +/- \$1,053) and 25,598 ( +/- \$1,686) respectively $(\mathrm{p}=<0.0001)$. There was also significantly higher reimbursement for rehab / Skilled Nursing Facility and hospital / inpatient care for the patients undergoing a posterior approach in both private payer (Figure 1) and Medicare databases (Figure 2). In all cohorts within this study, the hospital related reimbursement more than doubled the surgeon related reimbursement.

Conclusion: This study compares resource utilization for a 90-day episode of care for anterior and posterior approaches for CSM surgery. There is a statistically significant higher resource utilization for patients undergoing posterior approach for CSM. Understanding reimbursement patterns for anterior vs. posterior approaches for CSM will help providers design a bundled payment for patients requiring surgery for CSM. The data also suggests that hospital related reimbursement is the major driver of payments.

Presentation \#18. Trends in Resource Utilization and Rate of Cervical Disc Arthroplasty and Anterior Cervical Discectomy and Fusion throughout the United States from 2006 to 2013

Comron Saifi, MD, New York, NY; Arielle W. Fein, BA, New York, NY; Alejandro Cazzulino, BA, New York, NY; Alex Ha, MD, New York, NY; Ronald A. Lehman Jr., MD, New York, NY; K. Daniel Riew, $M D$, New York, $N Y$

Introduction: Given the increasing national focus on healthcare utilization and value-based care, spine surgeons, administrators, and policy makers must compare not only health based outcomes but also 
resource consumption in the management of spinal pathology. The purpose of this study was to compare anterior cervical discectomy and fusion (ACDF) to cervical disc arthroplasty (CDA) from 2006 - 2013 throughout the United States with regard to national incidence, hospital costs, length of stay (LOS), routine discharge, and revision burden.

Materials and Methods: Patient data from the National Inpatient Survey (NIS) database for primary ACDF, revision ACDF, primary CDA, and revision CDA from 2006 - 2013 were included in this study. Demographic and economic patient data were determined for the following ICD-9 CM codes: 81.02, 81.32, 84.62, and 84.66, respectively. The NIS database represents a $20 \%$ sample of discharges from U.S. hospitals and was weighted to provide national estimates. Revision burden was defined as the ratio of revision procedures to the sum of primary and revision procedures.

Results: An estimated 1,059,403 primary ACDF and 13,099 primary CDA surgeries were performed in the U.S. from 2006 to 2013 $(\mathrm{p}<0.0001)$. The annual total number of surgeries for both ACDF and CDA showed a gradual increase over the 8-year period. The annual number of ACDF surgeries increased $5.7 \%$ in a non-linear fashion from 120,617 in 2006 to 127,500 in 2013 (mean per year: 132,425; range: $120,617-147,966)$. The annual total number of CDA surgeries increased $190 \%$ in a similarly non-linear fashion from 540 in 2006 to 1,565 in 2013 (mean per year: 1,637; range: 540 - 2,381) (Figure 1).

Demographic data were compared demonstrating that the CDA patients tended to be younger and have private insurance or 'other' insurance, which includes worker's compensation, compared to the ACDF patients $(p<0.0001)$. Mean LOS was longer for ACDF than for CDA (ACDF: mean 2.3 days, range: $2.2-2.4$; CDA: 1.5 days, range: $1.3-2.0 ; \mathrm{p}<0.0001)$. The mean percentage of patients with routine discharge was significantly higher in the CDA group (ACDF: $89 \%$, range $86-92 \%$; CDA: $96 \%$, range $94-98 \%$; p-value $<0.0001$ ). The overall mean hospital costs per ACDF procedure was \$16,178, significantly more expensive than the overall mean cost per CDA of $\$ 13,197(\mathrm{p}$-value $=0.0007)$ (Figure 2).

The CDA mean revision burden, at 5.9\% (range: $3.9-7.4 \%$ ), was greater than the ACDF mean revision burden of $2.3 \%$ (range: $2.1-$ $2.8 \%)(p$-value $=0.01)$. The mean cost of revision ACDF remained steady and averaged $\$ 19,270$ (range: $\$ 17,423-\$ 21,256$ ). The mean cost of revision CDA was significantly less with a mean of $\$ 14,153$ (range: $\$ 11,723-\$ 16,555)$ ( $\mathrm{p}$-value $<0.0001)$.

Conclusion: In this large, national cohort, patients who underwent CDA experienced lower hospital costs, shorter length of stay, and a higher rate of routine discharges than patients who underwent ACDF. However, the CDA revision burden $(5.9 \%)$ was more than double compared to $\mathrm{ACDF}(2.3 \%)$. Following an initial steep increase in CDA from 2006 to 2009 , CDA procedures have decreased nearly $400 \%$ more than ACDF procedures over the same period. Additionally, the ratio of ACDF to CDA in the U.S. was 81:1. Given that studies have found CDA to be more cost-effective than ACDF, further research is needed on the relative decline of CDA from a national health care cost perspective.

\section{Presentation \#19. Impact of Type of Graft on Patient Reported Outcomes and Costs following Anterior Cervical Discectomy and Fusion}

Silky Chotai, MD, Nashville, TN; Scott L. Parker, MD, Nashville, TN; Elliott Kim, MD, Nashville, TN;Ahilan Sivaganesan, MD, Nashville, TN; Matthew J. McGirt, MD, Charlotte, NC; Clinton J. Devin, MD, Nashville, TN; J. Alex Sielatycki, MD, Nashville, TN

Background: Autograft harvested from iliac crest, variety of allografts, and structural cage including polyetheretherketone (PEEK) interbody spacer remains the most common graft choices for ACDF surgery. The impact of type of graft on outcomes and costs continues to be a debate. We set out to determine the patient reported outcomes (PROs), cost, complications, readmission and RTW associated with the autograft, allograft and PEEK.

Methods: Patients undergoing elective ACDF for degenerative cervical conditions were enrolled into prospective longitudinal registry. Patient-reported outcomes were recorded at baseline, and 24-months postoperatively. Hospital discharge and billing records were collected prospectively. Bivariate analyses were conducted to compare the PROs, cost of surgery, 90-day complications, readmission and return to work (RTW) following ACDF surgery with autograft vs. PEEK vs. allograft.

Results: Total 260 patients were included in the analysis. ACDF with autograft was performed in 69 patients $(26.5 \%)$, allograft in 25 patients $(9.6 \%)$ and PEEK spacer in 166 patients $(63.8 \%)$. There was no significant difference in change scores at postoperative 24-month for NDI, NRS-NP, and NRS-AP and EQ-5D between the groups. The patients undergoing autograft $(4.2 \pm 19.6)$ and PEEK $(4.2 \pm 16.3)$ had higher improvement in SF-12 PCS compared to those undergoing ACDF with allograft $(-9.3 \pm 19.9)(P=0.002)$. There were no significant differences in cost of surgery (autograft: $\$ 14683 \pm \$ 6841$ vs. PEEK: $\$ 14,410 \pm \$ 6,386$, vs. allograft: $\$ 1,4261 \pm \$ 7,870, P=0.27$ ), 90-day complications (autograft: $n=4,5.8 \%$, PEEK: $n=7,4.2 \%$, allograft: $\mathrm{n}=2,8 \%, \mathrm{P}=0.47$ ), readmission (autograft: $\mathrm{n}=4,5.8 \%$, PEEK: $\mathrm{n}=5,3 \%$, allograft: $\mathrm{n}=0, \mathrm{P}=0.51$ ) and RTW (autograft: $\mathrm{n}=$ $33,47.8 \%$, PEEK: $n=74,44.5 \%$ and allograft: $n=9,36 \%, P=0.59$ ).

Conclusion: ACDF with autograft or PEEK had higher improvement in general physical health compared to ACDF with allograft. The patients undergoing ACDF with autograft had higher length of hospital stay, however the overall cost of surgery did not differ significantly between the groups. In the current era of valuebased reimbursements, the choice of graft might not influence the cost and outcomes following ACDF surgery, as previously thought.

\section{Presentation \#20. Factors Associated with Financial Relationships between Spine Surgeons and Industry: An Analysis of the Open Payments Database}

Joseph A. Weiner, BS, Chicago, IL; Ralph W. Cook, BS, Chicago, IL; Sohaib Z. Hashmi, MD, Chicago, IL; Michael S. Schallmo, BS, Flossmoor, IL; Danielle S. Chun, BA, Chicago, IL; Kathryn A. Barth, BA, Chicago, IL; Sameer K. Singh, BA, Chicago, IL; Alpesh A. Patel, $M D, F A C S$, River Forest, IL; Wellington K. Hsu, MD, Chicago, IL

Introduction: Over the past decade, there has been growing media perception that financial relationships between physicians and industry influence clinical judgment, potentially undermining patient care. This has the potential to blemish the fragile relationship between patients and the medical community - one that is grounded in the ethical principal of beneficence. Although there have been a number of studies that have utilized the Open Payments database, it is a cumbersome conglomeration of individual payments and difficult to conceptualize. We aimed to present the most recent Open Payments data as it applies to orthopaedic and neuro spine surgeons in a comprehensible format, providing metrics to better understand how demographic characteristics - including type of surgeon, years in practice, type of practice, type of medical degree, type of graduate, gender, and region of practice - may be associated with both the quantity and magnitude of industry-surgeon payments.

Methods: A comprehensive database of 5,898 spine surgeons in the United States with corresponding data of industry payments from 2013 - 2014 were derived from the Open Payments website. Demographic data for each surgeon was collected including the type of residency training each surgeon completed, years of experience since last formal training, practice setting, type of medical degree, place of training, gender, and the region of practice. Generalized linear mixed models using a Beta distribution with a logit link were utilized to determine the relationship between demographics and industry payments.

Results: A total of 5,898 spine surgeons practicing in the United States who performed spine fusion on Medicare patients from 2011 
- 2013 met inclusion criteria for this study. In this dataset, $91.6 \%$ of surgeons within our cohort reported at least one financial relationship with industry. The median number of payments from industry to surgeon over the reporting period was 14 , worth a median total value of $\$ 994.07$ (Table 1). Surgeons receiving over $\$ 1,000,000$ from industry during the reporting period represented $6.6 \%$ of the database and accounted for $83.5 \%$ of the total value exchanged. Demographic factors associated with increased median industry payments included: orthopaedic training $(\mathrm{p}<0.001)$, academic practice setting $(\mathrm{p}<$ $0.0001)$, male gender $(\mathrm{p}<0.0001)$, and West or South region of practice $(p<0.0001)$. Linear regression analysis revealed a strong inverse relationship between years of experience and number of payments from industry $(\mathrm{r}=-0.967, \mathrm{p}<0.0001)$ (Figure 1).

Conclusions: Financial relationships between spine surgeons and industry are highly prevalent. A small subset of high earning spine surgeons in our cohort received very large payments, which accounted for a majority of the total transactional value provided by industry. Surgeon demographics (practice setting, gender, years in practice, type of training, and geographic region of practice) have a significant impact on financial relationships with industry.

\section{Presentation \#21. Cervical Sagittal Imbalance is Associated with a Higher Rate of Reoperation for Adjacent Segment Disease following Anterior Cervical Discectomy and Fusion}

Matthew W. Colman, MD, Chicago, IL Dustin H. Massel, BS, Northbrook, IL Benjamin C. Mayo, BA, Chicago, IL William W. Long, BA, Willoughby, OH Krishna D. Modi, BS, Schaumburg, IL Kern Singh, MD, Chicago, IL

Introduction: Adjacent segment disease (ASD) following anterior cervical discectomy and fusion (ACDF) is relatively common, and is the result of several proposed etiologies including increased biomechanical stresses on adjacent motion segments and the natural course of disc degeneration. Despite the evolving understanding of regional and global sagittal alignment, the effect of sagittal alignment on adjacent level breakdown following an ACDF remains unknown. Therefore, the purpose of this study was to evaluate cervical sagittal alignment parameter effect in ASD following an ACDF.

Methods: A retrospective case-control study was performed using a prospectively maintained surgical database of patients who underwent 1 - or 2-level ACDF by a single-surgeon. Patients who underwent reoperation for ASD following index ADCF were identified and compared to matched controls with an uncomplicated postoperative course. The ASD and control cohorts were analyzed with regard to baseline demographics and comorbidity burden, operative characteristics, patient reported outcomes, arthrodesis rate of the index procedure, and cervical sagittal alignment parameters. Differences in patient demographics and preoperative characteristics were assessed using independent sample t-tests and Chi-squared tests. The association between cervical sagittal alignment parameters and ASD reoperation was analyzed using Poisson regression with robust error variance (binary outcomes) or multivariate linear regression (continuous outcomes) adjusted for preoperative characteristics.

Results: A total of 435 patients who underwent 1- or 2-level ACDFs were included in the analysis, with an overall reoperation rate for ASD of 2.1\% $(\mathrm{N}=9)$. Patients who underwent reoperation for ASD had slightly higher preoperative Visual Analogue Scale (VAS) pain scores $(7.0 \pm 2.2$ vs. $5.4 \pm 1.7, p=0.047)$ compared to the controls. All other preoperative characteristics were no different between cohorts (Table 1). Patients who required reoperation for ASD had a higher baseline C2-C7 Sagittal Vertical Axis (SVA) (30.3 \pm 8.6 vs. $22.2 \pm 8.4, \mathrm{p}=0.048$; Table 2). There were no differences with regard to C2-7 Cobb Angle, T1 Slope, or Cranial Tilt. Additionally, there was a higher rate of pseudoarthrosis at the index level in the ASD cohort $(22.2 \%[\mathrm{~N}=2]$ vs. $0.0 \%[\mathrm{~N}=0], \mathrm{p}=0.141$; Table 2$)$ compared to the controls, however this was not statistically significant.
Conclusions: Regional sagittal malalignment in the cervical spine may be associated with a higher rate of adjacent segment breakdown following ACDF. In the current study, C2-7 SVA was the most predictive parameter for requiring a reoperation, and should be evaluated with other contributing factors in prognostic and risk-based discussions for ASD. Future studies are necessary to determine the effect of overall global sagittal imbalance on regional cervical parameters and the risk of reoperation for ASD following ACDF.

\section{Presentation \#22. A Novel Score Predicting Spine Sagittal Imbalance Based on a Lateral Cervical Plain Radiograph}

Ezequiel Goldschmidt, MD, PhD, Pittsburgh, PA; Federico Angriman Sr., MD, Boston, MA; Bruno Ferreyro, MD, Buenos Aires, Argentina; Nitin Agarwal, MD, Pittsburgh, PA; Zachary J. Tempel, MD, Pittsburgh, PA; Peter C. Gerszten, MD, MPH, Pittsburgh, PA; Adam S. Kanter, MD, Pittsburgh, PA; David O. Okonkwo, MD, PhD, Pittsburgh, PA; Peter G. Passias, MD, Westbury, NY; Justin K. Scheer, MD, Chicago, IL; Themistocles S. Protopsaltis, MD, New York, NY; Virginie Lafage, PhD, New York, NY; Frank J. Schwab, MD, New York, NY; Robert Shay Bess, MD, Rye, NY; Christopher P. Ames, MD, San Francisco, CA; Christopher I. Shaffrey, MD, Charlottesville, VA; D. Kojo Hamilton, MD, ISSG, Pittsburgh, PA

Introduction: Sagittal imbalance is recognized as a significant variable that contributes to spinal deformity. Clinical outcomes after spine surgery are known to correlate with sagittal balance (SB). SB is traditionally measured by the C7-S1 plumb line as demonstrated on a 36-inch long-cassette film. Abnormal positive alignment induces compensatory changes within the cervical spine, including increased cervical lordosis and T1 slope. Patients presenting clinically with cervical pathology are not routinely assessed with long-cassette films. A validated tool that could determine the likelihood of overall spine malalignment using cervical radiographs alone would be of significant clinical and cost saving value.

Methods: A retrospective review of 930 patients that were part of awwcluding demographics, cervical lordosis, and T1 slope were analyzed. Patients were randomized in a 2:1 fashion into either a derivation cohort or a validation cohort.

Results: Of the 930 patients, 384 (41.3\%) had a positive SB. The final score for predicting SB greater than $+50 \mathrm{~mm}$ included: BMI $>$ 25 ( 1 point), age $>55$ years ( 2 points), and T1 slope $>27 \mathrm{o}$ ( 2 points). A score of $\geq 3$ had a specificity of $63.6 \%$ (CI $58.7-68.3 \%$ ) and a sensitivity of $82.9 \%$ (CI 77.6-87.3\%). The ROC area under the curve was 0.82 (CI95\% $0.78-0.85)$ and $0.81(\mathrm{CI} 95 \% 0.76-0.86)$ in the derivation and validation cohorts, respectively.

Conclusions: This large multicenter study internally validated a simple score to assess SB based upon cervical radiographs, BMI, and age alone. The preoperative awareness of abnormal SB in patients with cervical pathology might change surgical treatment and clinical outcomes. Patients with high scores would benefit from long-cassette film evaluation.

Presentation \#23. Relationship between T1 Slope and Cervical Alignment following Multi-level Posterior Cervical Fusion Surgery: Impact of T1 Slope Minus Cervical Lordosis

Seung-Jae Hyun, MD, PhD, Seongnam, Republic of Korea; Kim Ki-Jeong, MD, PhD, Seongnam, Republic of Korea

Study Design: Retrospective study.

Objective: (1) To assess the relationship between sagittal alignment of the cervical spine and patient- reported health-related quality-of-life (HRQOL) scores following multilevel posterior cervical fusion and

(2) to explore whether an analogous relationship exists in the cervical spine using T1 slope C2-C7 lordosis ('T1S CL'). 
Summary of Background Data. A recent study demonstrated that, similar to the thoracolumbar spine, the severity of disability increases with sagittal malalignment following cervical reconstruction surgery.

Methods: From 2007 - 2013, 38 consecutive patients underwent multilevel posterior cervical fusion for cervical stenosis, myelopathy, and deformities. Radiographic measurements included: (1) C0-C2 lordosis, (2) C2-C7 lordosis, (3) C2-C7 sagittal vertical axis (SVA), (4) T1 slope, and (5) T1S CL. Pearson correlation coefficients were calculated between pairs of radiographic measures and HRQOL.

Results: C2-C7 SVA positively correlated with neck disability index (NDI) scores $(r=0.495)$. C2-C7 lordosis $(P=0.001)$ and T1SCL $(\mathrm{P}=0.002)$ changes correlated with NDI score changes after surgery. For significant correlations between C2-C7 SVA and NDI scores, regression models predicted a threshold $\mathrm{C} 2-\mathrm{C} 7 \mathrm{SVA}$ value of $50 \mathrm{~mm}$, beyond which correlations were most significant. The T1S CL also correlated positively with C2-C7 SVA and NDI scores $(r=0.871$ and $\mathrm{r}=0.470$, respectively).

Results of the regression analysis indicated that a C2-C7 SVA value of $50 \mathrm{~mm}$ corresponded to a T1S CL value of $26.1^{\circ}$.

Conclusions: This study showed that disability of the neck increased with cervical sagittal malalignment following surgical reconstruction and a greater T1S CL mismatch was associated with a greater degree of cervical malalignment. Specifically, a mismatch greater than $26.1^{\circ}$ corresponded to positive cervical sagittal malalignment, defined as C2-C7 SVA greater than $50 \mathrm{~mm}$.

\section{Presentation \#24. Outcomes of Operative Treatment for Adult Cervical Deformity: A Prospective Multicenter Assessment with One-Year Follow-up}

Justin S. Smith, MD, PhD, Charlottesville, VA; Christopher I. Shaffrey, MD, Charlottesville, VA; Han Jo Kim, MD, New York, NY; Gregory M. Mundis, MD, San Diego, CA; Munish C. Gupta, MD, St. Louis, MO; Eric O. Klineberg, MD, Sacramento, CA; Frank J. Schwab, MD, New York, NY; Virginie Lafage, PhD, New York, NY; Renaud Lafage, BS, New York, NY; Peter G. Passias, MD, Westbury, NY; Themistocles S. Protopsaltis, MD, New York, NY; Brian J. Neuman, MD, Baltimore, MD; Alan H. Daniels, MD, Providence, RI; Tamir Ailon, FRCSC, MD, MPH, Charlottesville, VA; Justin K. Scheer, BS, Chicago, IL; Khaled M. Kebaish, MD, Baltimore, MD; Robert A. Hart, MD, Portland, OR; Michael O'Brien, MD, Plano, TX; Douglas C. Burton, MD, Kansas City, KS; Vedat Deviren, MD, San Francisco, CA; Todd Albert, MD, New York, NY; K. Daniel Riew, MD, New York, NY; Robert Shay Bess, MD, Rye, NY; Christopher P. Ames, MD, San Francisco, CA; International Spine Study Group, Brighton, CO

Introduction: Despite the potential for profound impact of adult cervical deformity (ACD) on function and health-related quality of life, there remains a paucity of high-quality studies that assess outcomes of surgical treatment for these patients. Our objective for the present study was to provide a prospective multicenter assessment of 1-year outcomes following surgical treatment for ACD.

Materials and Methods: Surgically treated ACD patients eligible for 1-yr follow-up were identified from a prospectively collected multicenter database with consecutive enrollment. Baseline deformity characteristics, surgical parameters, and 1-year outcomes were assessed. Standardized outcomes measures included Neck Disability Index (NDI, range $0-100)$, neck pain numeric rating scale (NRS) score (range $0-10$ ), and EQ5D-3S index (range $0-1$ ) and subscores (range $1-3$ ). Paired sample t-tests were used to compare 1-year and baseline measures.

Results: Of 77 ACD patients, 55 (71\%) had 1-year follow-up (64\% women, mean age 61 yrs, mean Charlson Comorbidity Index $[\mathrm{CCI}]$ of 0.6 , previous cervical surgery in $44 \%$ ). Diagnoses included: cervical sagittal imbalance $(62 \%)$, cervical kyphosis $(60 \%)$, proximal junctional kyphosis $(8 \%)$, and coronal deformity (10\%). Posterior fusion was performed in $85 \%$ (mean number of vertebral levels $=$
10 ), and anterior fusion was performed in $29 \%$ (mean number of vertebral levels $=5$ ). Three-column osteotomy was performed in $24 \%$ of patients. Mean operative time was $6.5 \mathrm{hrs}$ and mean estimated blood loss was $0.9 \mathrm{~L}$. At 1-year following surgery, ACD patients had significant improvement in NDI (50.5 to $38.0, \mathrm{p}<0.001)$, neck pain NRS (6.9 to 4.3, p < 0.001), EQ5D index (0.51 to $0.66, \mathrm{p}<0.001)$, and EQ5D

subscores: mobility (1.9 to $1.7, \mathrm{p}=0.019)$, usual activities (2.2 to 1.9 , $\mathrm{p}=0.007)$, pain/discomfort ( 2.4 to

$2.1, \mathrm{p}<0.001)$, anxiety/depression (1.8 to $1.5, \mathrm{p}=0.014)$. A nonsignificant trend favoring improvement was observed for EQ5D self-care $(1.5$ to $1.3, p=0.070)$. Compared with patients that achieved 1-year follow-up, those lost to follow-up did not differ significantly with regard to age, gender, CCI, number of fused anterior or posterior vertebral levels, or baseline NDI, neck pain NRS, or EQ5D scores.

Conclusions: Adult cervical deformity can produce significant pain and disability. Based on a prospective multicenter series of adults with cervical deformity, surgical treatment provided significant improvement in multiple measures of pain and function, including the NDI, neck pain NRS score, and EQ5D. Further follow-up will be necessary to assess the durability of these surgical procedures and the resulting improved outcomes.

Presentation \#25. Postoperative Cervical Sagittal Realignment after Debridement and Reconstruction in Cervical Spinal Tuberculotic Kyphosis

Kai Cao, MD, PhD, Nanchang, China; Jiaquan Luo, Nanchang, China; Zhimin Pan, MD, Nanchang, China; Junlong Zhong, Nanchang, China; Yiwei Chen, Nanchang, China; Pingguo Duan, Nanchang, China; Li Zhiyun, MD, Nanchang, China

Introduction: Cervical TB is not a rare disease in developing countries and can erode vertebra which results in the development of cervical kyphosis and myelopathy. However, no literature reported the correlation of the cervical spine realignment after debridement and reconstruction surgery with the improvement of HROQLs.

Methods: Forty-six kyphotic cervical tuberculosis (TB) cases were included in this study. Preoperative and 2-year follow-up radiological parameters were measured, including $\mathrm{C} 0-2 \mathrm{Cobb}$ angle, $\mathrm{C} 2-7 \mathrm{Cobb}$ angle, C2-7 SVA, center of gravity to C7 SVA (CG-C7 SVA), thoracic inlet angle (TIA), T1 slope (TS), neck tilt (NT). NDI was recorded to analyze the improvement of HROQLs. The correlation between cervical alignment and NDI were analyzed. CT scans was used to assess the bone fusion after surgery.

Results: Forty-three cases showed bone fusion on CT scan, the fusion rate was $93.5 \%$. The preoperative $\mathrm{C} 0-2 \mathrm{Cobb}$ angle, $\mathrm{C} 2-7 \mathrm{Cobb}$ angle, TS, TIA, was $-27.9 \pm 10.6^{\circ}, 16.8^{\circ} \pm 5.2^{\circ}, 15.8 \pm 8.1^{\circ}$,

$62.7^{\circ} \pm 15.8^{\circ}$, improved to $-22.9 \pm 4.2^{\circ}(\mathrm{P}<0.01),-16.1^{\circ} \pm 7.5^{\circ}(\mathrm{P}$ $<0.01), 21.8 \pm 7.3^{\circ}(\mathrm{P}<0.01)$,

$70.7^{\circ} \pm 12.6^{\circ}(\mathrm{P}<0.05)$, but the pre- and postoperative values of NT had no significant change $(\mathrm{P}>0.05)$. The preoperative $\mathrm{C} 2-7 \mathrm{SVA}$, CG-C7 SVA was $38.1 \pm 6.7 \mathrm{~mm}, 46.5 \pm 8.3 \mathrm{~mm}$, and improved to 10.2 $\pm 5.8 \mathrm{~mm}(\mathrm{P}<0.01), 20.5 \pm 6.2 \mathrm{~mm}(\mathrm{P}<0.01)$, respectively (Figure1). The preoperative NDI was

$33.6 \pm 5.1$, improved to $16.7 \pm 4.6(\mathrm{P}<0.01)$. NDI was significantly correlated with $\mathrm{C} 0-2$ Cobb angle, C2-7 Cobb angle, TS, TIA, C2-7 SVA and CG-C7 SVA.

Conclusion: Debridement and cervical reconstruction can make kyphotic tuberculotic cervical spine realigned normally, and meanwhile significantly improve the HROQLs. 
Presentation \#26. Management of Hangman Variant Fractures of the Axis

Thomas E. Niemeier, MD, Birmingham, AL; Sakthivel R. Manoharan, MD, Johnson City; Steven M. Theiss, MD, Birmingham, AL

Introduction: Traumatic spondylolisthesis of the axis with the fracture extending into the vertebral body has been incompletely characterized. Small case series have demonstrated high rates of neurological injury and cite difficulty treating closed due to greater instability secondary to extensive ligamentous injury. We hypothesize that this fracture pattern has minimal risk of ligamentous injury and can be adequately treated with closed methods.

Methods: Acute C2 fractures were identified retrospectively at a Level I trauma center from 2004 to 2015 from ICD-9 coding and confirm with three dimensional imaging. Fractures that displayed separation of the axis body from the posterior arch such that one or both vertically oriented fracture defects involved the posterior cortex of the axis body were classified as hangman variants. Displacement was determined based on horizontal displacement of the $\mathrm{C} 2$ vertebral body as well as angulation between the $\mathrm{C} 2-3$ vertebras.

Results: 107 hangman's variant fractures (14.5\%) were identified from a database of 735 acute $\mathrm{C} 2$ fractures. Average age on presentation was 54 years with over $90 \%$ occurring secondary to high-energy blunt trauma. Forty-four percent sustained other spine fractures including $35 \%$ with cervical spine or occipital condyle fractures. 106 of the 107 patients displayed no neurologic injury related to the cervical spine. One patient with a widely displaced fracture $(>8 \mathrm{~mm}$ ) sustained a complete neurologic injury that did not recover despite surgical stabilization. 90 patients received treatment in either hard collar orthosis or halo (14 underwent surgery and 3 deaths) with $83 \%$ followed as outpatients for an average of 32 weeks and median of 12 weeks. All patients treated in halo or hard collar demonstrated horizontal translation of less than $5 \mathrm{~mm}$ and C2-3 angulation of less than 15 degrees. MRI obtained in 29 patients $(32 \%)$ treated nonoperatively showed no evidence of C2-3 disk or ligamentous injury. No patients treated in halo or hard collar sustained late neurologic injury, progression of displacement or instability ( $>2 \mathrm{~mm}$ change in displacement or $>5$ degree change of C2-3 angulation) on follow-up radiographs. No difference was observed in radiographic outcome between patients treated in a hard collar or halo orthosis.

Conclusion: While widely considered a difficult fracture to treat with closed means, hangman variants are relatively neurologically benign injuries with low incidence of ligamentous injury. Fractures with less than $5 \mathrm{~mm}$ of horizontal translation and 15 degrees of angulation can be treated nonoperatively without the necessity of MRI. Our results suggest no advantage of halo immobilization versus hard collar orthosis.

\section{2nd Place Clinical Paper Award • \$1,000}

Presentation \#27. Risk Factors for Failure of Non-operative Treatment for Unilateral Cervical Facet Fractures

Carola F. Van Eck, MD, PhD, Pittsburgh, PA; Mitchel Fourman, MD, Pittsburgh, PA; Amir M. Abtahi, MD, Salt Lake City, UT; Louis Alarcon, MD, Pittsburgh, PA; William F. Donaldson III, MD, Pittsburgh, PA; Joon Y. Lee, MD, Pittsburgh, PA

Introduction: Approximately 5\% of subaxial cervical spine fractures involve isolated non-displaced facet fractures without spinal cord injury. Despite being relatively common, no consensus exists with regards to the optimal management of these injuries, and the failure rate and predictors of failure after conservative management remain unknown. The aims of this study were to determine the clinical failure rate with non-operative management of isolated unilateral subaxial facet fractures, to determine the percentage of patients who develop radiographic spondylolisthesis during follow-up, and to identify risk factors for clinical failure and spondylolisthesis.

Methods: This study was a retrospective review of the trauma registry at a Level I trauma center. All patients evaluated between 2002 and 2014 with isolated unilateral subaxial cervical facet fractures who underwent initial non-operative management were included in this study. All patients were treated in a hard cervical collar with frequent clinical and radiographic follow-up.

Computed tomography (CT) scans were used to define the level and pattern of the fracture and to measure fracture displacement, angle, spondylolisthesis, and percentage of the facet height and area involved in the fracture (Figure 1). Radiographic spondylolisthesis was defined as greater than $2 \mathrm{~mm}$ on the initial CT scan and greater than $10 \%$ of the anterior-posterior dimensions of the inferior vertebral endplate on follow-up radiographs.

Results: 74 patients were included in the study. Mean follow-up was 9 months $+/$ - 22 months (0-121 months). Fracture characteristics are listed in Table 1.

$7 / 74$ patients $(9 \%)$ underwent surgery during follow-up. In patients who underwent surgical intervention, time between injury and surgery ranged from 2 to 10 weeks. Procedures included 5 anterior cervical discectomy and fusions (ACDF) $(62.5 \%), 2$ posterior cervical fusions $(25 \%)$, and 1 laminectomy (12.5\%). Risk factors for failure of conservative management included presence of radiculopathy at the time of presentation, higher body mass index (BMI), increased Injury Severity Score (ISS), greater initial fracture displacement, and more than $2 \mathrm{~mm}$ of spondylolisthesis on the initial post-injury CT scan.

15 / 74 patients $(20 \%)$ had spondylolisthesis greater than $10 \%$ on follow-up radiographs. Risk factors for presence of spondylolisthesis included higher BMI, higher Charlston comorbidity score, greater initial fracture displacement, and greater percentage of the facet height involved in the fracture. Only $2 / 15(13 \%)$ of patients with spondylolisthesis on follow-up imaging developed radicular symptoms and none developed myelopathy or catastrophic neurologic deterioration.

Conclusion: The clinical failure rate of isolated unilateral subaxial cervical facet fractures in the present study $(9 \%)$ was significantly lower than that reported in previous studies. Despite a $20 \%$ rate of radiographic spondylolisthesis on follow-up imaging, only a small percentage of patients developed radicular symptoms and none developed catastrophic neurologic deterioration. The results of this study, therefore, suggest that patients with non- or minimally displaced facet fractures who do not have neurological symptoms at the time of presentation can safely and often successfully be managed conservatively with careful observation and follow-up.

Presentation \#28. An Economic Case for the Surgical Treatment of Type-II Odontoid Fractures in the Elderly: A Markov CostUtility Analysis based on the Prospective AOSpine Geriatric Odontoid Fracture Study

Jefferson R. Wilson, MD, PhD, Toronto, ON, Canada; James S. Harrop, MD, Philadelphia, PA; Gregory D. Schroeder, MD, Philadelphia, PA; Alexander Vaccaro, MD, PhD, Philadelphia, PA; Jens R. Chapman, MD, Seattle, WA; Srinivas Prasad, MD, MS, Philadelphia, PA; Justin S. Smith, MD, PhD, Charlottesville, VA; Christopher Kepler, MD MBA, Philadelphia, PA; Paul M. Arnold, MD, Kansas City, KS; Michael G. Fehlings, MD, PhD, Toronto, ON, Canada

Introduction: Type-II odontoid fractures are the most common cervical fractures encountered in the elderly, with an overall incidence that appears to be rising. Substantial uncertainty continues to surround optimal management of these injuries; while non-operative treatment is associated with a high rate of non-union, surgery is more costly and may be associated with high complication rates in this age group. To provide further evidence on this topic, we performed a value based 
assessment comparing costs and health gains between these treatment strategies.

Methods: We constructed a Markov cost-utility model, with a life-long time horizon, comparing quality-adjusted survival and costs of surgical vs. non-operative treatment (external orthosis), from the perspective of the payer, for the base case of a 75 year-old person with a type-II odontoid fracture. Mean utility values, corresponding to the health states of interest, were calculated from primary data (SF-6D scores) prospectively collected during the AOSpine GOF Study. Probability rates for mortality, complications, failure / fusion were estimated based on a systematic review of the literature. Per patient treatment costs, presented in 2016 US dollars, were obtained from the Healthcare Cost and Utilization Project, National Inpatient Sample, averaged over a 7-year period $(2003-2010)$. Incremental Cost Effectiveness Ratios (ICERs) were evaluated relative to a Willingness to Pay (WTP) threshold of 50,000USD / QALY. Oneand two-way sensitivity analyses were performed to identify threshold values for age, cost, utility and probability values. Finally, probabilistic sensitivity analysis, using Monte Carlo Simulation with 1,000 sample iterations, was performed to generate an ICER scatterplot and costeffectiveness acceptability (CEA) curve.

Results: Over a lifetime, as compared to non-operative treatment, surgery was associated with an average gain of an additional 0.81 QALYs and additional costs of 12,788USD, resulting in an ICER of $15,725 \mathrm{USD} / \mathrm{QALY}$ for the base case analysis. With increasing age, surgery became less cost-effective, with age 96 representing the threshold beyond which the ICER exceeded the WTP threshold (ICER at age 85:26,069USD/QALY; ICER at age 95:46,049USD/QALY). Results were also found sensitive to variation in year 1 post-op mortality rates, with surgery becoming less cost-effective as surgical mortality increased and as non-operative treatment morality decreased (Figure 1). Model results were less sensitive to variation in costs or fusion and complication rates for each strategy. Probabilistic sensitivity analysis revealed surgery to be the most cost-effective strategy in $79.3 \%$ of the 1000 iterations sampled, as depicted in the ICER scatterplot (Figure 2). Generation of CEA curve demonstrated surgery to the preferred strategy above a WTP threshold of 20,000USD.

Conclusion: Surgical treatment for type-II odontoid fractures in the elderly appears to provide better value with respect to costs and health gains as compared to non-operative management with external orthosis alone. However, surgery becomes less cost effective with increasing patient age and increasing probability of early postop death. This implies that while surgery is likely to be the preferred approach for the younger healthier patient, conservative management may be more appropriate for the older patient with a higher probability of short-term mortality. Further studies are needed to confirm the findings presented here.

\section{Presentation \#29. Minimally Clinical Important Difference (MCID) of a Clinical Impairment Measure Specific for Traumatic Tetraplegia: A Multi-Centre Assessment of the GRASSP Version} 1.0

Sukhvinder Kalsi-Ryan, MSc, PhD, PT, Toronto, ON, Canada; Michael G. Fehlings, MD, PhD, Toronto, ON, Canada

Introduction: GRASSP Version 1.0 is a clinical impairment measure designed specifically to assess the upper limb after traumatic cervical spinal cord injury (SCI). The GRASSP consists of 5 subtest scores that characterize the upper limb; it captures subtle changes in neurological impairment during the acute, sub-acute, and chronic phases of recovery. Psychometric properties of reliability validity, responsiveness and minimally detectable difference are established.

The remaining psychometric property to be established is Minimally Clinical Important Difference (MCID), which is required to establish use in efficacy and interventional studies. The objectives of this study were to: 1) Establish the MCID values for the GRASSP and 2) To summarize how the GRASSP can be applied in clinical / interventional trials as a tool to define effectiveness of new therapies.

Methods: A prospective longitudinal study including 53 individuals with acute traumatic cervical SCI was conducted as a multi-centre study. Serial testing consisted of GRASSP, International Standards for Neurological Classification for Spinal Cord Injury (ISNCSCI) and a patient questionnaire designed to acquire the patients perception of change over time were administered 0 to 10 days, $1,3,6$, and 12 months post injury. Analysis: Using a validated anchor-based approach patients rated their status as same, better and much better related to the specific domains of the GRASSP, the mean change in GRASSP scores was calculated for these groups.

Results: 53 individuals sustaining a traumatic cervical SCI with NLI ranging from $\mathrm{C} 2$ to $\mathrm{T} 1$ (AIS $\mathrm{A}=11, \mathrm{~B}=5, \mathrm{C}=16, \mathrm{D}=23$ ) at baseline. MCID and minimally important different (MID) values for the group of individuals perceiving their upper limb impairment to be much better are presented in Table 1. Three calculations were done, anchor-based, standard deviation and standard error of measure.

Conclusion: MCID of the GRASSP has been established and can be a useful measure to establish efficacy of interventions as well as meaningfulness of the change as it relates to the patient. Although, MCID remains to be an elusive psychometric property, the benefit of an available value / s contributes to the investigators understanding of the treatment effect. We recommend application of these MCID values for group-level analysis when conducting research and interpreting data examining groups of patients as opposed to assessing individual patients. These MCID values may provide a basis for sample size calculations for future investigation using the GRASSP.

\section{Presentation \#30. Principal Radiographic Characteristics for Cervical Spinal Deformity: A Health-Related Quality of Life Analysis}

Hongda Bao, MD, PhD, Nanjing, China; Jeffrey Varghese, BS, New, York, NY; Renaud Lafage, New, York, NY; Barthelemy Liabaud, MD, Bassel Diebo, New, York, NY; Subaraman Ramchandran, MBBS, MS, New, York, NY; Louis Day, Brooklyn, NY; Cyrus Jalai, BA, New, York, NY; Dana Cruz, MD, Bronx, NY; Thomas J. Errico, MD, New, York, NY; Themistocles S. Protopsaltis, MD, New York, NY; Peter G. Passias, MD, Westbury, NY; Aaron Buckland, FRACS, New, York, NY; Frank J. Schwab, MD, New, York, NY; Virginie Lafage, PhD, New York, $N Y$

Introduction: Despite that cervical kyphosis was traditionally recognized as the presentation of cervical deformity, increasing studies demonstrated that cervical kyphosis may not imply definitely cervical deformity and may be a potential mechanism to maintain horizontal gaze. Therefore several other criteria for cervical deformity should be investigated, especially based on health-related quality of life (HRQOL). This study aims to propose radiographic characteristics of patients with cervical disability and to investigate the relevant parameters when assessing cervical alignment.

Methods: Patients (pts) with normal thoracolumbar alignment (T1 pelvic angle $<15^{\circ}$ ), Neck Disability Index (NDI) and no prior cervical surgery were included. Pts were stratified into cervical asymptomatic (Asymp: $\mathrm{NDI} \leq 15$, VAS neck $\leq 3$ and VAS arm $\leq 3$ ) or symptomatic (Symp: NDI $>15$ or VAS neck $>3$ or VAS arm $>3$ ) groups. Sagittal parameters including SLS (Slope of Line of Sight), McGS (McGregor Slope), and CC (C2-C7 cervical curvature) were compared between groups. Logistic regression and principle component analysis (PCA) were performed to distinguish cervical symptomatic pts.

Results: There were 171 Patients (mean 44 y / o) included, with groups Asymp N = 64 and Symp N = 107. Symp pts were older (35y vs. 50y; $\mathrm{p}<0.001)$ and had worse NDI (5.4 vs. 41.3, p <0.001). C2-C7 SVA, McGS and SLS were significantly different between groups (all $\mathrm{p}<0.05)$, while CC was comparable $(\mathrm{p}=0.09)$. Logistic regression revealed that C2-C7 SVA $(\mathrm{OR}=1.043, \mathrm{p}=0.049)$ and SLS $(\mathrm{OR}=$ 
0.936, $\mathrm{p}=0.029)$ were independent risk factors for poor HRQOL. Using PCA, the equation $0.55 \times \mathrm{C} 2 \mathrm{C} 7 \mathrm{SVA}+0.34 \times \mathrm{C} 0 \mathrm{C} 2$ angle + $0.77 \times \mathrm{CC}$ was calculated and showed significant correlations with NDI, VAS-Arm, VAS-Back and EQ5D scores $(r=0.30,0.26,0.24$ and 0.28 , respectively). ROC analysis revealed improved predictability of regression and PCA formulas formula for HRQOL compared to single radiographic parameters.

Conclusion: $\mathrm{CC}$ alone is unable to distinguish different cervical HRQOL status and thus should not be regarded as the only criteria for CSD. Gaze parameters should be integrated in evaluation of HRQOLdefined CSD, although the predictability of gaze parameters is lower than that of cervical alignment. This data supports the integration of both gaze and alignment parameters into cervical spinal deformity classification.

\section{Presentation \#31. Diffusion Tensor Imaging Can Predict Surgical Outcomes of Patients with Cervical Compression Myelopathy}

Mitsuhiro Kitamura, Chiba, Japan; Satoshi Maki, MD, Chiba, Japan Takeo Furuya, MD, PhD, Chiba, Japan; Yasushi Iijima, Chiba, Japan; Junya Saito, Chiba, Japan; Masashi Yamazaki, MD, PhD, Tsukuba, Japan; Masao Koda, MD, PhD, Chiba, Japan

Introduction: Surgical decompression is often recommended for symptomatic cervical compression myelopathy (CCM). It is important to know the prognosis of surgical outcomes and to recommend appropriate timing for surgery. Diffusion tensor imaging (DTI) can be used to evaluate patients with CCM quantitatively because it can provide microstructural information regarding the spinal cord with quantitative diffusion parameters. The objective of this study was to assess whether preoperative DTI parameters can predict surgical outcomes of patients with CCM.

Methods: We enrolled 20 patients with CCM who had undergone surgery and were followed up for more than 6 months. Japanese Orthopaedic Association (JOA) score for cervical myelopathy was evaluated before and 6 months after surgery. Surgical outcomes were measured by both change and recovery rate of JOA score, and were regarded as good if change in JOA score was 3 points or higher or the recovery rate of JOA score was $50 \%$ or higher. The patients were examined using a 3.0 T magnetic resonance system before surgery. For DTI acquisitions, reduced field of view (rFOV) diffusion-weighted spinecho single-shot echo-planar imaging was used. rFOV is a new method that enables acquisition of high- resolution DTI. Regions-of-interest were determined based on the geometry of the cord on the B0 map and DTI parameters were measured using DTIStudio software (Johns Hopkins Medical Institute, Johns Hopkins University). Measured DTI parameters were fractional anisotropy (FA), mean diffusivity (MD), axial diffusivity (AD), and radial diffusivity (RD). The correlations between DTI parameters and surgical outcomes were analyzed. For statistical analysis, a Mann - Whitney U test and Spearman correlation coefficient were used. The predictive performance of FA value for good surgical outcomes was evaluated by the area under the receiver operator characteristic (ROC) curve. $\mathrm{p}<0.05$ was considered significant.

Results: JOA score was 8.9 preoperatively and 11.6 at 6 months after surgery and improved significantly $(p<0.001)$. Change of JOA score moderately correlated with FA $(\mathrm{r}=0.51, \mathrm{p}=0.02)$. Moreover, the recovery rate of JOA score correlated moderately with FA $(\mathrm{r}=$ $0.49, p=0.03)$. Change of JOA score and the recovery rate of JOA score tended to correlate with $\mathrm{AD}(\mathrm{r}=0.41, \mathrm{p}=0.07$ and $\mathrm{r}=0.43, \mathrm{p}$ $=0.06$, respectively), but this tendency was not significant. Change of JOA score and the recovery rate of JOA score did not correlate with $\mathrm{MD}$ and $\mathrm{RD}$. The area under the ROC curve for prognostic precision for surgical outcomes evaluated by change and recovery rate of JOA score were 0.76 and 0.89 , respectively, indicating good model prediction by FA. The cut-off value of FA for predicting good surgical outcomes evaluated by change and recovery rate of JOA score were 0.60 and 0.57 , respectively.
Conclusion: It is feasible to predict surgical outcomes of patient with CCM using DTI. DTI can be used as an imaging biomarker for surgical prognosis of CCM patients.

Presentation \#32. Posterior Cervical Spinal Cord Shift following Posterior Decompression and Prediction of Persistent Anterior Spinal Cord Compression using K-Plane: A Three Dimensional Modification of K-Line on MRI

Sang-Hun Lee, MD, PhD, Seoul, Republic of Korea; Ki-Tack Kim, MD, Seoul, Republic of Korea; Jung-Hee Lee, MD, PhD, Seoul, Republic of Korea; Kyung-Chung Kang, MD, Seoul, Republic of Korea

Introduction: Posterior spinal cord shift (PS) following posterior cervical decompressive surgery plays a key role in recovery of myelopathy, and persistent anterior spinal cord compression (AC) is a well-known factor related to unfavorable prognosis. Although a K-line or modified $\mathrm{K}$-line, connects $\mathrm{C} 2$ and $\mathrm{C} 7$ central canal, is useful parameters to predict insufficient decompression after laminoplasty (LP), these concepts only considered the midsagittal images. In addition, there have been no studies on characteristics of PS and difference between LP and laminectomy with fusion (LF). The purpose of this study is to evaluate related factors of PS and predict AC using the authors' novel K-plane, a three dimensionally modification of K-line.

Materials and Methods: We retrospectively reviewed preoperative and follow-up cervical spine MR images of patients underwent LP or LF for cervical myelopathy. The changes of C27 angles by Cobb method, PS and midsagittal diameter of the spinal cord at the level of maximal compression, and alignment of the center of spinal cord were measured on MR images and their correlations were analyzed. K-plane was decided either $(+)$ or $(-)$ by the combination of sagittal $\mathrm{K}$-line ( + : any part of the line posterior to spinal canal, $\mathrm{N}$ : within spinal canal, -: anterior to spinal canal,) and coronal K-line (-: asymmetric cord compression, +: symmetric, Figure 1). The relationship between $\mathrm{K}$-plane and presence of $\mathrm{AC}$ was analyzed.

Results: A total of 62 patients $(\mathrm{M}: \mathrm{F}=43: 19$, mean age 58.9, CSM: OPLL $=56: 6, \mathrm{LP}: \mathrm{LF}=40: 22$, ) were enrolled. Mean time interval of follow-up MR was 2.2 months and mean number of the level decompressed was 3.97. C27 angle showed significant positive relationship with PS and kyphotic change of the spinal cord. Mean C27 angle changes ( $\mathrm{LP}: \mathrm{LF}=4.4$ vs. $\left.-2.5^{\circ}\right)$, mean PS $(2.6$ vs. $4.8 \mathrm{~mm})$ and mean spinal cord alignment change $\left(10.2\right.$ vs. $\left.17^{\circ}\right)$ were parameters showed significant differences between LP and LF groups. Mean changes of spinal cord midsagittal diameter showed no significant difference (1.7 vs. $2.2 \mathrm{~mm})$. The correlations between K-plane and presence of AC showed significance in LP group only (LP vs. LF: sensitivity $94.1 \%$ vs. $66.7 \%$, specificity $95.7 \%$ vs. $57.9 \%, p<0.001$ ) (Figure 2)

Conclusions: Lordotic cervical spine and laminectomy with fusion produced larger posterior shift and kyphotic change of the spinal cord alignment than kyphotic cervical spine and laminoplasty, respectively. A K-plane, three-dimensional modification of K-line, could be a useful guideline to predict persistent anterior spinal cord compression following laminoplasty. 
Presentation \#33. Modular Organization of Whole-Brain Resting-State Functional Connectivity in Spinal Cord Injury: A Comparative Study

Mayank Kaushal, Greenfield, WI; Akinwunmi Oni-Orisan, MD, Milwaukee, WI; Gang Chen, PhD, Wauwatosa, WI; Wenjun Li, PhD, Milwaukee, WI; Jack Leschke, Milwaukee, WI; Benjamin Kalinosky, PhD, Beaver Dam, WI; Matthew D. Budde, Milwaukee, WI; Brian D. Schmit, Milwaukee, WI; Vaishnavi Muqeet, MD, Milwaukee, WI; Shekar N. Kurpad, MD, PhD, Milwaukee, WI

Introduction: The application of graph theory to functional connectivity involves modeling the brain as a complex network comprising of nodes and edges. This allows for the inspection of whole brain connectivity patterns and the calculation of quantifiable network metrics for comparison between groups. To date, large-scale network analysis modeled on graph theory has not been applied to resting- state functional networks in complete spinal cord injury (SCI) patients. The purpose of the present study was to evaluate the topological architecture of the whole brain resting-state functional connectivity to characterize the pattern of modular reorganization in patients with cervical SCI both qualitatively and quantitatively.

Methods: After obtaining the necessary IRB approval, 15 subjects with chronic (duration $>2$ years) complete (ASIA A) cervical SCI and 15 neurologically intact controls were scanned. The data were preprocessed and then parcellated into 264 regions of interest (ROI). Correlation analysis was performed between the average time course obtained from each ROI for every possible ROI pair. The correlation values obtained resulted in the creation of an association matrix following which the threshold of 0 was applied to include only the positive correlation values. Then a modularity algorithm from brain connectivity toolbox (BCT) was applied to organize the data into modular patterns using MATLAB. Subsequently, statistical analysis was carried out to check for differences in the number of connections and the network density (cost) associated with those connections for a particular module between the SCI and the control groups using the two sample t-test.

Results: The modular organization pattern for the control and the SCI groups is shown in Figure 1. Both groups assembled into 4 distinct modules, namely the default mode network (DMN), salient network (SN), executive control network (ECN), and sensorimotor network (SMN). Upon visual inspection of individual modules, qualitative differences were noted in the number and the membership of constituent ROIs comprising a particular module. Quantitative comparison showed a decrease in the SCI group for all four modules in terms of the number of connections. Further, the network density (cost), defined as the number of connections present divided by the total number of possible connections also showed significant reduction in the SCI group for each of the 4 modules at cost threshold of 0.35 (Figure 2).

Conclusion: The demonstration of modular organization pattern in both the SCI and the control groups highlights the applicability of largescale network analysis modeled on graph theory for the evaluation of complex brain networks. The modules showed reductions for both the number of connections and the network density associated with those connections. The decrease might be due to reduced efficiency of information processing within specialized regions of the brain owing to the distortion in the transmission of input impulses from downstream neural structures. In addition, the alterations to modules in terms of the number and the membership of constituent ROIs is indicative of underlying neural plasticity of the cortical structures.
Presentation \#34. Prospective Clinical and Radiographic Assessment of the Cervical Spine in Professional Rodeo Riders after Exposure to Greater than 10G Linear Acceleration

Robert Trigg McClellan, MD, San Francisco, CA; Alexander A. Theologis, MD, San Francisco, CA; Jeremy D. Shaw, MS, MD, San Francisco, CA; Jeffrey Mulvihill, MD, San Francisco, CA; Musa Zaid, MD, Palo Alto, CA; Safa Herfat, PhD, San Francisco, CA; Christopher Hess, MD, PhD, San Francisco, CA; Jared Narvid, MD, San Francisco, CA; Alisa Gean, MD, San Francisco, CA; Jeremie Larouche, MD, San Francisco, $C A$

Introduction: Whiplash Associated Disorder (WAD) remains a controversial clinical diagnosis. Few models exist to comprehensively assess the clinical effects of rotational and linear acceleration on the cervical spine. As professional rodeo riders are subjected to repeated flexion / extension events during their rides, they represent an ideal population to assess the clinical and radiographic effects of massive acceleration and deceleration on the cervical spine. The purpose of this study is to evaluate the clinical and radiographic effect of a measurable acceleration / deceleration event on the cervical spine in professional rodeo riders using objective clinical data and validated health related quality of life (HRQoL) scores.

Materials and Methods: Adult ( $>18$ years) professional rodeo riders were prospectively evaluated. After informed consent, each subject before their ride underwent focused physical examination by a licensed physician and completed the Visual Analogue Score (VAS) for Neck, Arm, and Back pain, the Neck Disability Index (NDI), the Short Form-36 (SF-36), and EuroQuol (EQ-5D). VAS neck, arm, and back pain scores were also assessed post-ride. Six riders also underwent pre- and post-event MRIs of the cervical-spine. Peak linear accelerometer data were recorded by a mouth-guard accelerometer. Descriptive statistics were performed and pre- and post-ride data were compared using Student's T-tests with set at $\mathrm{p} \leq 0.05$.

Results: Twenty-one male professional riders (bareback-8, saddle bronc-7, bull-6; average age

$24.3 \pm 5.6$ years) were enrolled. They reported to have competed in an average of $55 \pm 25$ rodeos per year. The minority reported a prior neck injury (5/21) and missing rodeos due to neck injuries (4/21). No riders reported prior neck surgery.

Baseline NDI $(4.9 \pm 6.5)$, EQ-5D $(0.89 \pm 0.15)$, and SF-36 (PCS $51.9 \pm 6.2$, MCS $55.1 \pm 4$ ) were recorded.

Seventeen riders' mouth-guards recorded events $>10 \mathrm{~g}$. Mean linear acceleration was $23.8 \pm 13.9 \mathrm{~g}$. Peak linear acceleration was $62.8 \mathrm{~g}$. Post-ride VAS for neck pain trended towards higher scores relative to pre-ride scores, although the difference was not significant (pre: 0.48 vs. post: $1.0 ; p=0.10$ ). Post- ride VAS scores for arm pain were not significantly different from pre-ride scores $(p>0.25)$. There were no differences in post-ride VAS scores between the bareback, saddle bronc, and bull riding groups $(\mathrm{p}>0.20)$. Mild disk bulging adjacent to pre-existing disease was noted in 2 / 6 post-ride MRI scans, but no clinically significant changes were identified.

Conclusion: Repeated high G-forces did not significantly impact the clinical incidence of neck, arm, or back pain in professional rodeo riders, nor did they produce any significant MRI changes. In this population, the G-forces experienced by the cervical spine did not produce any acute cervical symptoms or new neurological deficits. As the G-forces experienced by these riders are significantly greater than most activities of daily living and low-speed rear-end automobile collisions, these data provide a clinically useful context for evaluating patients with whiplash injuries. 
Presentation \#35. Anterior Cervical Discectomy and Fusion with Stand-Alone PEEK Cages with Integrated Screws Compared to an Allograft and Plate Construct

Grant D. Shifflett, MD, New York, NY; Jahanzeb Kaikaus, BS, Chicago, IL; Melissa G. Goczalk, BS, Chicago, IL; Bryce A. Basques, MD, Chicago, IL; Phil K. Louie, MD, Chicago, IL; Frank M. Phillips, $M D$, Chicago, IL

Introduction: Traditionally anterior cervical plate (ACP) fixation with structural allograft has been used for reconstruction after anterior decompression of the cervical spine. More recently, stand-alone (SA) cages with integrated screw fixation have been popularized for cervical reconstruction; however, there is little comparative data between these two procedures.

Materials and Methods: Consecutive patients who underwent either SA or ACP procedures for one level degenerative pathology performed by one surgeon at a single institution between 2011 and 2013 were evaluated. Plain lateral radiographs of the cervical spine were performed pre-operatively, immediately post-operatively, and at final follow-up. The following radiographic parameters were assessed: pre-operative disc height, T1 slope, focal lordosis, overall cervical lordosis, C2-C7 sagittal vertebral angle (cSVA), and interbody cage subsidence. Symptomatic pseudarthrosis and reoperation rates were recorded.

Results: Sixty-two patients were included in the study (33 ACP and 29 SA). Average follow-up was 16.8 months. There were no significant differences in baseline demographic variables including age, sex, obesity, smoking, or level of operation (Table 1). Both constructs were equally effective at restoring local and segmental lordosis $(\mathrm{p}>0.05)$ (Table 2). The SA construct was as effective as the allograft- ACP construct in terms of restoration of disc height, focal or global lordosis, and cSVA. There was no statistical difference ( $\mathrm{p}$ $>0.05$ ) between the average immediate post-operative height of the surgical segment $(35.2+/-3.7 \mathrm{~mm})$ when compared to the height of the surgical segment at final follow-up $(34.1+/-3.7 \mathrm{~mm})$ for all patients. Subsidence (defined as $>3 \mathrm{~mm}$ loss of overall surgical segment height from immediate post-operative to final follow-up) occurred in $12.1 \%$ of ACP cases and $13.7 \%$ of SA constructs $(p>0.05)$. There were no significant differences in symptomatic pseudarthrosis rates requiring reoperation between the two groups at final follow-up. Independent risk factors for reoperation were male sex $(p=0.049)$ and smoking $(\mathrm{p}=0.017)$.

Conclusion: These results indicate that SA anterior cervical discectomy and fusion results in equivalent radiographic alignment parameters when compared to ACP constructs with no difference in pseudarthrosis or reoperation rates. SA constructs generally are faster to apply and are often entirely intra-discal which may reduce swallowing difficulties associated with prominent ACPs. In addition SA constructs are less likely to impinge on adjacent level discs compared to ACPs. This information is useful for surgical decisionmaking and informing future studies that seek to further identify differences between these two procedures.

Presentation \#36. Anterior Cervical Decompression and Fusion - Why Do Patients Proceed to Surgery? Does it Matter? Is it Neck Pain, Arm Pain or Neurological Change that Motivates the Patient?

Eduardo C. Beauchamp, MD, Minneapolis, MN; Timothy A. Garvey, $M D$, Minneapolis, $M N$

Introduction: The goal of an adequate surgical evaluation is to document objective evidence of specific anatomic lesions that when treated surgically, yield predictable and measurable patient perceived beneficial outcomes. A known consensus exists regarding surgical interventions for common cervical spine disorders such as radiculopathy, cervical myelopathy or traumatic cervical instability, but for patients with axial neck pain as the major complaint, the surgical indications are less clear.

Due to this uncertainty, we are hesitant on intervening surgically on patients who present with a chief complaint of mainly neck pain, but many of these patients, after further evaluation, are diagnosed with other pathologies rather than axial neck pain. Our objective was to assess the patient's specific motivation and primary reason for seeking care (neck pain, arm pain, both) and compare it to the physician's surgical diagnosis and to determine if the patient's driving factor had any effect on the surgical outcome.

Materials and Methods: IRB approved retrospective chart review of patients who underwent single or two level primary anterior cervical decompression and fusion at our institution in a two year period were analyzed. From the database 213 patients were identified who met the inclusion criteria for the study and who had completed an extensive outcome questionnaire regarding pain and self function at a 1-year follow up. Patient perceived outcomes were measured using NDI and VAS scores. Physician perceived outcome was determined using Odom's criteria. Descriptive summaries were generated overall and by patient chief complaint category.

Results: 213 patients were eligible for the study. Of these, 53 (24.9\%) had a chief complaint of arm pain, 112 (52.6\%) complained of neck pain, $46(21.6 \%)$ complained of equal arm and neck pain, and $2(0.9 \%)$ had other complaints (imbalance, weakness). No statistical difference on demographic data was noted among chief complaint groups except for worker's compensation or disability status (38.4\% of patients with initial neck pain, $18.9 \%$ of those with predominant arm pain and $28.3 \%$ of neck = arm pain, $\mathrm{p}<0.05$ ).

Average decrease in VAS for all patients was 3.6 for arm VAS and 3.4 for neck VAS which meets the minimum clinically important difference. The arm pain group had a mean decrease of 5.2 and 2.3 in arm and neck VAS respectively, while the neck group decrease was 2.2 and 3.7 as well. NDI decrease was similar among groups. Physician perceived outcomes using Odom's criteria were excellent to good in $88.6 \%$ of the patient with primarily arm pain and $74.1 \%$ in those with mainly neck pain, but this difference was not statistically significant $(\mathrm{p}>0.05)$ Table 1.

Conclusion: Patients perceived improvement with surgical management, as compared with their pre- operative status, regardless of whether they experienced mainly neck or arm pain as a chief complaint. Patients presenting with primarily neck pain may experience similar results in both physician and patient perceived outcomes to those who present with primarily arm pain.

Presentation \#38. Longitudinal Effects of Intraoperative Neurophysiological Monitoring on Costs and Clinical Outcomes for Single Level Cervical Spine Surgery

John P. Ney, MD, MPH, Bedford, MA; Daniel P. Kessler, JD, PhD, Standford, $C A$

Introduction: Cervical spinal surgery carries serious potential neurological complications, including radiculopathy and permanent spinal cord injury. Intraoperative neuromonitoring may mitigate these complications. We investigate the effects of IONM on cost and quality of care for a large sample of cervical spine patients in a retrospective cohort study.

Methods: We used IMS's PharMetrics Plus Health Plan Claims data to identify commercially-insured patients aged 18-63 with singlelevel cervical spine surgery in an inpatient setting from $2008-2012$. We observed patients for 6 months before and 12 months after the date of admission for their index surgery. We used the 6-month preindex baseline period to measure patients' health status; we used the 12-month followup to evaluate post-index economic and clinical outcomes. We calculated four types of outcomes: total spending, neurological complications, readmissions, and outpatient opiate use 
(as a proxy for pain). We constructed a "treatment" variable to indicate whether patients received IONM during their index surgery. We also constructed variables to control for characteristics of patients' health status and demographics, ancillary services received during their index admission, and other characteristics of patients' illness and surgery. We estimated regression models of the effects on outcomes of IONM, holding constant these covariates plus fixed effects for patients' 3 digit zip code and year of procedure.

Results: Holding other factors constant, IONM was associated with increased spending for the index surgery of $\$ 1,229(p=0.001)$, but decreased spending post-discharge of $\$ 1,615(\mathrm{p}=0.010)$, for a net effect of $-\$ 386(\mathrm{p}=0.608)$ in the year after the index admission. Shorter length of stay $(0.116$ days, $p=0.004)$ and fewer readmissions (20.5 per thousand, $\mathrm{p}=0.036$ ) accounted for at least part of the post-discharge savings. The reduction in readmissions occurred in the month after the index admission and persisted through the entire following year. IONM was associated with decreased rates of nervous system complications (of 4 per thousand, $\mathrm{p}=0.048$ ) and post-discharge opiate use (of 17 prescriptions per thousand, $p=0.050$ ) in the year after the index admission.

Conclusions: Holding other factors constant, IONM was approximately cost-neutral in a sample of nonelderly commerciallyinsured patients with uncomplicated cervical spine surgery, and was associated with improved patient health outcomes. Understanding the extent to which IONM has similar effects in other populations is an important topic for future research.

\section{Presentation \#39. Surgical and Functional Outcomes after Multi- Level Cervical Fusion for Degenerative Disc Disease Compared to Fusion for Radiculopathy: A Study of Workers' Compensation Population}

Mhamad Faour, MD, Cleveland, OH; Joshua T. Anderson, BS, Cleveland, OH; Nicholas U. Ahn, MD, Cleveland, $\mathrm{OH}$

Introduction: Cervical fusion provides more than $90 \%$ of symptomatic relief for radiculopathy and myelopathy. However, cervical fusion for degenerative disc disease (DDD) without radiculopathy or myelopathy is considered controversial. In addition, multi-level fusion is associated with poorer surgical outcomes with increased levels fused. The objectives of this study is to 1) evaluate presurgical and surgical factors that are associated with poor return to work status after multi-level cervical fusion, 2) compare outcomes after multi-level cervical fusion for patients with degenerative disc disease without radiculopathy or myelopathy (discogenic pain) versus patients with radiculopathy.

Methods: Data of cervical comorbidities was collected from Ohio Bureau of Workers' Compensation for subjects with work-related injuries using "International Classification of Diseases, Ninth Edition (ICD-9)" and "Current Procedural Terminology" (CPT) codes. See Figure 1 for patients' selection flow diagram. Multivariate logistic regression was performed to evaluate whether stable RTW status was achieved or not. Surgical and functional outcomes were compared between groups.

Results: In the study population of patients who underwent multilevel cervical fusion, multiple factors were negatively associated with stable RTW status within 3 years after fusion: Fusion for discogenic pain (DDD without radiculopathy) (OR:0.74; 95\%CI: 0.56-0.98: $\mathrm{p}<0.05$ ), age older than 55 (OR:0.41; 95\%CI: 0.30-0.56: $\mathrm{p}<0.05$ ), opioid use prior to surgery (OR:0.60; 95\%CI: $0.48-0.75$ : $\mathrm{p}<0.05$ ), initial psychological evaluation prior to surgery (OR:0.35; $95 \% \mathrm{CI}$ : $0.24-0.52$ : $\mathrm{p}<0.05$ ), injury-to-surgery $>2$ years (OR:0.68; $95 \% \mathrm{CI}$ : $0.54-$ 0.82 : $\mathrm{p}<0.05$ ), and instrumentation (OR:0.68; 95\%CI: $0.53-0.88$ : $\mathrm{p}<0.05)$.

Stable RTW status was achieved in $43.3 \%$ of the DDD group and $54.8 \%$ of the Radiculopathy group (p: 0.0001 ). DDD patients were less likely to achieve stable RTW status after surgery compared to patients with radiculopathy $(\mathrm{OR}=0.63[0.50-0.79])$. RTW rate within the first year after surgery was achieved in $33.6 \%$ of the DDD group and $43.7 \%$ of the radiculopathy group (p: 0.0003 ). Compared to radiculopathy patients, DDD patients were less likely to RTW within one year after surgery $(\mathrm{OR}=0.65[0.52-0.82])$.

Higher rate of disability benefits were awarded to patients with DDD after surgery $(p=0.002)$. Higher postoperative opioid use was also observed in the DDD group (p: 0.001). Patients with DDD received opioids for longer than 8 weeks after surgery at a higher rate than patients with radiculopathy $(\mathrm{p}=0.003)$.

No significant difference in reoperation rate within 3 years after fusion was observed between the groups.

Conclusion: Multiple detriments affect stable return to work status after multi-level cervical fusion including DDD. Degenerative disc disease was associated with lower RTW rates, less likelihood to return to work, higher disability, and higher opioid use after surgery. Multi-level cervical fusion for degenerative disc disease without radiculopathy or myelopathy may be counterproductive. Future studies should investigate further treatment options of DDD, and optimize patient selection criteria for surgical intervention.

\section{Presentation \#40. An Analysis of Conflicts of Interest in Cervical Spine Surgery: The Effects of Industry Payments on Practice Patterns and Complication Rates}

Ralph W. Cook, BS, Chicago, IL; Joseph A. Weiner, BS, Chicago, IL; Michael S. Schallmo, BS, Flossmoor, IL; Danielle S. Chun, BA, Chicago, IL; Sameer K. Singh, BA, Chicago, IL; Kathryn A. Barth, BA, Chicago, IL; Alpesh A. Patel, MD, FACS, River Forest, IL; Wellington K. Hsu, MD, Chicago, IL

Introduction: Recent demands from the United States Congress and the Institute of Medicine have highlighted the importance of conflict of interest among physicians. Previous studies have identified orthopaedic and neurological surgeons as receiving among the highest industry payment amounts. However, to date, no study has investigated the association or potential effects of disclosed industry payments with quality of patient care. We sought to determine whether financial relationships with industry had any impact on practice patterns or complication rates of spine surgeons.

Methods: A comprehensive database of spine surgeons in the United States with compiled data of industry payments, fusion recommendation rates, and complication rates was created. Practice pattern data was derived from a publically available Medicare-based database generated from CPT codes, which included the total number of, and rate at which each spine surgeon recommends fusion (2011 - 2012). Complication rate data for cervical fusion procedures for each surgeon was extracted from the ProPublica Surgeon Scorecard database (2009 - 2013) (https://projects.propublica. org/surgeons/), which utilizes in-hospital mortality and readmission within 30 days of discharge for designated conditions as complications of surgery. A mixed-effects model adjusting for age and health- status along with hospital and surgeon random-effects was used to risk-adjust each surgeon's raw complication rate. Data regarding industry payments were derived from the Open Payments website (2013 - 2014) which collects information on "transfers of value" worth more than $\$ 10$ as reported by manufacturers and group purchasing organizations (https://www.cms.gov/OpenPayments/index.html). Surgeons performing fewer than 10 fusions from 2011-2012 and those without complication data were excluded from this study. Pearson correlation coefficients and multivariate regression analyses were used to determine the relationship between industry payments, cervical fusion recommendation rates, and complication rates.

Results: A total of 2,110 spine surgeons (54\% orthopedic, $46 \%$ neurosurgeon) practicing in the United States met our inclusion criteria. Pearson correlation analyses revealed a negligible relationship between industry payments and cervical fusion recommendation rates $(\mathrm{r}=$ 
$0.13 ; \mathrm{p}<0.01)$. An $\mathrm{r}$-value $>0.30$ is generally accepted as a threshold for weak correlations, with values lower than this designated as negligible. Multivariate regression analysis demonstrated no significant relationships among payments and cervical recommendation or complication rates. Additionally, a comparison of 2007 surgeons receiving payments from industry and 103 surgeons without disclosed payments revealed no significant differences between the two groups with regard to fusion recommendation or complication rates.

Conclusions: While spine surgeons receive the highest industry payment amounts among all subspecialties, conflict of interest does not appear to have a significant impact on practice patterns or complication rates.

\section{Presentation \#41. The Effect of Surgeon Volume on Complications, Length of Stay, and Costs after Anterior Cervical Fusion}

Bryce A. Basques, MD, Chicago, IL; Philip K. Louie, MD, Chicago, IL; Grant D. Shifflett, MD, New York, NY; Dustin H. Massel, BS, Northbrook, IL; Benjamin C. Mayo, BA, Chicago, IL; Daniel D. Bohl, MD, MPH, Chicago, IL; Kern Singh, MD, Chicago, IL

Introduction: Increased surgeon volume may be associated with improved outcomes following surgical procedures. However, there is a lack of information on the effect of surgeon volume on short-term outcomes following anterior cervical fusion (ACF). The purpose of the present study was to identify the association between surgeon volume and inpatient complications, length of stay, and costs associated with ACF.

Materials and Methods: A retrospective cohort study of ACF patients was performed using the Nationwide Inpatient Sample (NIS) from 2003 to 2009. Surgeon volume was divided into three categories, volume less than the 25 th percentile of surgeon volume, between 25 th and 74th percentile of surgeon volume, and greater than or equal to the 75 th percentile of surgeon volume. Multivariate regression was used to compare the rates of adverse events, hospital length of stay, and total hospital costs between surgeon volume categories.

Results: A total of 419,212 ACF patients were identified. The 25th percentile for volume was 5 cases per year, and the 75th percentile for volume was 67 cases per year. Average age was $51.7 \pm 11.6$ (mean \pm standard deviation) and $52.9 \%$ of patients were female. Volume $<25$ th percentile was associated with increased rates of any adverse event (OR 3.8, $\mathrm{p}<0.001)$, and multiple individual complications including death (OR 2.5, p = 0.014), pneumonia (OR 4.4, p < 0.001), sepsis (OR $2.4, \mathrm{p}<0.001$ ), surgical site infection (OR 4.1, $\mathrm{p}<0.001$ ), and wound dehiscence (OR 3.5, p < 0.001) on multivariate analysis (Table 1). Notably, volume $\geq 75$ th percentile was associated with decreased rates of any adverse event (OR $0.7, p<0.001)$, death (OR $0.6, p=0.028)$, sepsis (OR 0.5, p <0.001), and wound dehiscence (OR 0.6, p < 0.001$)$.

Average length of stay was $1.7 \pm 2.2$ days, and the average hospitalization cost was $\$ 12,999 \pm \$ 9,058$. On multivariate analysis, length of stay was significantly increased by 2.3 days $(\mathrm{p}<0.001)$ for surgeons below the 25th percentile of volume and was decreased by 0.3 days for surgeons with volume $\geq 75$ th percentile (Table 2 ). Hospital costs were $\$ 4,569$ more for surgeons with $<25$ th percentile of volume and $\$ 1213$ less for surgeons with $\geq 75$ th percentile volume.

Conclusion: In this nationally representative sample, surgeons with volume less than the 25 th percentile had significantly increased complications, length of stay, and costs. Conversely, surgeons with $\geq$ 75th percentile volume had decreased of complications, length of stay, and costs. These results indicate that patients and healthcare systems may derive significant benefits from using surgeons that perform a high annual volume of ACF procedures. Presentation \#42. Defining Health Utility following One- or Two-
Level ACDF or CDR at Five Years

Steven J. McAnany, MD, Atlanta, GA; Samuel C. Overley, MD, New York, NY; Jun Sup Kim, MD, New York, NY; Robert Brochin, MD, New York, NY; Sheeraz A. Qureshi, MD, MBA, New York, NY

Introduction: In the era of comparative value analysis, there is a tremendous emphasis to consider the relative costs and benefits of procedures that are performed for the same indication. Anterior cervical discectomy and fusion (ACDF) and cervical disc replacement (CDR) are two procedures that can be performed for the treatment of acute cervical disc herniation with myelopathy/radiculopathy. A critical component of the cost-effectiveness analysis remains the ability to properly define value. The purpose of this study is to determine the utility of one- and two-level ACDF and CDR at various post-operative time points.

Materials and Methods: Data from the Medtronic one- and twolevel Prestige Cervical Disc investigational device exemption (IDE) studies. Data from the 36-item Short Form Health Survey (SF-

36) were collected at baseline, 12 months, 24 months, 36 months, and 60 months post-operatively. Using the SF-6D algorithm, the SF-36 scores were converted into utility scores for each time point. A repeated measures ANOVA was used to compare to detect overall differences between related means. Tukey's method for multiple comparisons was used to determine which means within the groups were statistically different.

Results: Table 1 summarizes the calculated utility values for oneand two-level ACDF and CDR for each of the time points. There was a statistically significant difference between groups as determined by repeated measures one-way ANOVA $(\mathrm{F}(2,9)=15.63, \mathrm{p}=0.0008)$ (Figure 1). A Turkey post-hoc analysis indicated that one-level ACDF had a statistically lower utility score at all time points when compared with one- and two-level CDR ( $p=0.04, p-002)$ (Table 2). Similarly, two-level ACDF was shown to have a lower utility values at all time points when compared with two-level CDR $(p=0.010)$. One-level ACDF and two-level ACDF were not shown to have different utility values at any time point $(p=0.55)$. One-level CDR and two-level CDR did not differ in their utility values at any time point $(p=0.67)$.

Conclusions: The health utility values for one- and two-level $\mathrm{ACDF}$ and $\mathrm{CDR}$ were calculated for the pre-operative baseline state, 12 month, 24 month, 36 month, and 60 month post-operative state. Overall, CDR was found to have a higher health utility state for one- and two-level procedures at evert time point. One- and twolevel ACDF procedures did not differ in their health utility state at any time point. Similarly, one- and two-level CDR demonstrated the same health utility score at every time point. The results of this study indicate that CDR results in a higher post-operative health utility state than ACDF, though two- level CDR does not provide any significant additional health benefit compared to single-level.

\section{Presentation \#43. Patient Reported Outcomes and Costs in Revision Cervical Surgery}

Elliott J. Kim, MD, Nashville, TN; Silky Chotai, MD, Nashville, TN; Joseph B. Wick, BA, Nashville, TN; David P. Stonko, BS, MS, Nashville, TN; Ahilan Sivaganesan, MD, Nashville, TN; Clinton J. Devin, MD, Nashville, $T N$

Introduction: Revision rates for cervical spine surgery are steadily increasing. It is important to be able to counsel patients on expected results and financial burden following a revision procedure. However outcomes and cost of these procedures are poorly defined in the literature. The purpose of this study was to determine cost and utilize standardized outcome measures to assess the results of revision cervical spine surgery.

Materials and Methods: Patients undergoing revision cervical 
spine surgery at a single institution were included between October 2010 and January 2016 in a prospective registry database. Patients were divided into three cohorts depending on their etiology for revision surgery including recurrent disease, pseudoarthrosis, or adjacent segment disease. Patient reported outcomes (PROs) including Neck Disability Index (NDI), EuroQol-5D (EQ-5D), numeric rating scaleneck pain (NRS-NP), and numeric rating scale-arm pain (NRS-AP) were measured at baseline as well as 12 months following revision surgery. Mean costs at 12 months following revision surgery were also calculated. Satisfaction was determined by the NASS patient satisfaction index. Variables were compared using student t-test.

Results: A total of 115 patients (Table 1) underwent cervical revision surgery for recurrent disease $(\mathrm{n}=21)$, pseudoarthrosis $(\mathrm{n}=$ $45)$, and adjacent segment disease $(n=49)$. There was a significant improvement in all the patient-reported outcomes at 12 months (Figure 1) following surgery regardless of etiology $(p<0.0001)$. Total cost of revision surgery ranged between $21294 \pm 8614$ to $23914 \pm$ 15396 depending on pathology. No significant differences were seen between costs (Table 2) among different revision groups $(p=0.53)$. No differences were seen between 12 month PROs among the three revision groups with regards to NDI $(p=0.66), E Q-5 D(p=0.99)$, $\mathrm{NRS}=\mathrm{NP}(\mathrm{p}=0.72)$, or NRS-AP $(\mathrm{p}=0.47)($ Table 3$)$. Satisfaction was met in $75.5-85.7 \%(p=0.21)$ of patients depending on the etiology of the revision need. Complication rates were between $4-9 \%$.

Conclusion: This is one of the first studies to determine costs and outcome measures in the setting of cervical spine revision surgery. Significant improvement in PROs were seen 12 months following revision surgery regardless of etiology. The etiology of revision surgery did not affect the amount of improvement in outcome measures or cost. Satisfaction rates were similar among different groups as well ranging between $75.5-85.7 \%$. Based on our analysis a majority of patients can expect to receive some benefit by 12 months and are satisfied with their procedure. It is imperative to counsel patients and set expectations prior to undergoing revision procedures.

\section{Presentation \#44. Anterior Cervical Discectomy (ACDF): A More Exact, Non-traditional Activity and Resource Cost Accounting at University Center Shows \$16,500 Cost Differential}

Barton L. Sachs, MD, MBA, Charleston, SC; John Glaser, MD, Charleston, SC; Thomas S. Brehmer, MHA, Irmo, SC

Definition: Unsustainable healthcare expenditures in U.S. challenge providers and organizations to deliver higher quality care at lower cost with increased value. To accomplish this aim, we focused on our true costs. We compared time-driven activity-based costing (TDABC) to traditional accounting methodology (TA) at our university academic medical center (AMC) for primary anterior cervical discectomy (ACDF) over care cycle from admission surgery day to 90 days postsurgery. Direct and indirect costs from consecutive ACDF patients in FY'15 were identified from various hospital cost centers at our institution.

Data: The total cost per case using TA compared to TDABC method was $\$ 29,222$ versus $\$ 13,073$, respectively. We identified sizeable differences in cost estimates between TA and TDABC when comparing physicians and advanced practice provider (APP) personnel costs (\$894 vs \$501), non- physician personnel costs $(\$ 5,264 \mathrm{vs}$ $\$ 1,251)$, space and equipment costs $(\$ 1,207$ vs \$151), and indirect costs $(\$ 9,964$ vs $\$ 1,859)$. Implants $(\$ 5,126)$ and consumables $(\$ 4,173)$ costs were equivalent in both methods as these figures were based on hospital purchase price.

With TA, personnel accounted for $25 \%$ of overall costs, with physicians / APPs and non-physicians representing 4\% and 21\% of total costs, respectively. Space and equipment represented $16 \%$, consumables $14 \%$, and indirect costs $24 \%$ of overall costs. Using TDABC, personnel represented 14\% of overall costs (physicians / APP
$4 \%$, non-physicians $10 \%$ ), space and equipment $1 \%$, consumables $32 \%$, and administrative overhead $14 \%$.

Results: TDABC suggests that TA overestimates the personnel costs of ACDF by approximately

$\$ 7,400$ per patient. Additionally, TA may overestimate space, equipment, and indirect costs by more than $\$ 9,074$. TDABC offers patient-level granular cost data that is imperative in reducing costs and negotiating sustainable fixed-bundled payment contracts with payers. Concurrently, we improved value of longitudinal care for ACDF using new implant vendor contracts that reduced our implant cost by $24 \%$ $45 \%$.

\section{1st Place Basic Science Paper Award • \$2,000}

Presentation \#45. Directly Reprogrammed Human Neural Precursor Cells-A Novel and Translationally Relevant Source for Cell Replacement Therapy in Spinal Cord Injury

Narihito Nagoshi, MD, PhD, Toronto, ON, Canada; Jan-Eric Ahlfors, BS, MBA, MSc, Laval, QC, Canada; Mohamad Khazaei, PhD, Toronto, ON, Canada; Morio Matsumoto, MD, PhD, Tokyo, Japan; Masaya Nakamura, MD, PhD, Tokyo, Japan; Cindi Morshead, PhD, Toronto, ON, Canada; Michael G. Fehlings, MD, PhD, Toronto, ON, Canada

Introduction: Although induced pluripotent stem cells (iPSCs) have potential as a therapeutic strategy for spinal cord injury (SCI), several issues remain to be solved before they can be transferred to the clinic. These include challenges with karyotypic instability, the potential for tumorigenicity, issues surrounding their long-term differentiation potential and challenges with scaleability. To overcome these challenges, we have recently developed a novel approach to generate neural precursor cells which are directly reprogrammed (drNPCs) from human somatic cells without gene integration. The entire reprogramming process has an efficiency of $40 \%$ and takes less than 2 weeks; a significant improvement over the current iPSC technique which takes half a year with net efficiency of $1 \%$. Additionally, drNPCs can be expanded $>100$ fold in less than one month in vitro. The objective of this study is to determine the reparative/regenerative capacity and safety profile of drNPC transplantation following SCI.

Materials and Methods: Clip compression SCI was induced at T7 in athymic nude rats. Nine days post- injury, we transplanted 1) drNPCs, 2) pro-oligodendrocyte precursor cells (pro-OPCs) differentiated from drNPCs, and 3) vehicle into the injured spinal cord ( $\mathrm{n}=10$ per group). Functional assessments including BBB, CatWalk system, and Tailflick test were performed weekly. The animals were sacrificed nine weeks after SCI, and immunohistochemical analysis was performed by labeling the transplanted cells with HuN antibody. Histomorphometric analysis was performed with luxol fast blue and hematoxylin/ eosin staining. To evaluate tumorigenicity, the cells were transplanted into the intact spinal cord of immunodeficient NOD/SCID mice.

Results: Cell survival rates at nine weeks post-SCI were $28.89 \%$ and $24.14 \%$ in drNPC and pro- OPC groups, respectively. The engrafted pro-OPCs showed significantly more differentiation into APC + oligodendrocytes compared to drNPCs $(50.32 \%$ vs. $15.14 \%$, $\mathrm{p}<0.01$ ) (Figure 1A). In contrast, differentiation rates of $\mathrm{NeuN}+$ neurons and GFAP+ astrocytes showed no significant differences between the groups (Figure 1A). The transplanted pro-OPCs also differentiated into mature oligodendrocytes with pi-GST and MBP expression (Figure 1B-C). Electron microscopic examination showed transplanted pro-OPCs myelinated host axons with thick lamellar structure. Histomorphometric analysis showed significantly increased white matter area and reduction of lesion area in the pro-OPC group compared to the other groups (Figure 2A-B). Motor function in the pro-OPC group was significantly improved in BBB scores and the CatWalk system (stride length and swing speed) compared to SCI 
injured rats that received vehicle only (Figure 2C-E). The tail-flick test to measure thermal allodynia showed no significant difference among the groups. At 150 days post spinal cord injection of cells in NOD/SCID mice, all mice remained healthy and with no histological evidence of tumorigenicity.

Conclusions: drNPC-derived OPCs 1) promoted sparing of white matter and suppressed the expansion of the lesion, 2) contributed to motor neurological recovery without occurrence of allodynia, and 3) did not cause tumors in long-term follow-up. Additionally, the proOPCs predominantly differentiated into mature oligodendrocytes that myelinated host axons. For clinical application, oligogenic human drNPCs are safe and promising cell sources with the potential for tissue preservation and functional improvement after SCI.

\section{2nd Place Basic Science Paper Award • \$1,000}

Presentation \#46. Therapeutic Impact of Human Induced Pluripotent Stem Cell Derived Neural Progenitor Cells for the Treatment of Cervical Spinal Cord Injury

Hiroaki Nakashima, MD, Nagoya, Japan; Mohamad Khazaei, Toronto, ON, Canada; Anna Badner, Toronto, ON, Canada; Jonathon Chio, Toronto, ON, Canada; James Hong, BS, PhD, North York, ON, Canada; Narihito Nagoshi, MD, PhD, Toronto, ON, Canada; Kajana Satkundrarajah, MD, Toronto, ON, Canada; Chris Ahuja, MD, Toronto, ON, Canada; Andras Nagy, Toronto, ON, Canada; Michael G. Fehlings, MD, PhD, Toronto, ON, Canada

Introduction: Spinal cord injury (SCI) is a catastrophic event, however available treatment options remain limited. Cell replacement strategies have shown promise for recovery following SCI. Induced pluripotent stem cells (iPSCs) are a clinically relevant cell source that can be used to deliver patient- specific cellular therapy, avoiding immunological and ethical issues. Previous studies have shown that iPSCs derived from neural progenitor cells (NPCs) hold particular promise for the treatment of SCI. NPCs are multipotent, and are thus able to differentiate into neurons, oligodendrocytes and astrocytes. However, there are limited studies on the mechanism of action of human iPSCs (hiPSCs), and no studies evaluating the optimal differentiation state of hiPSCs. In addition, although the majority of the SCI population incurs cervical injuries, the efficacy of stem cells has not been extensively tested in cervical SCI. The aim of this study was to compare the regenerative therapeutic capacity of these oligogenic vs. neurogenic NPCs derived from hiPSCs in the setting of cervical SCI.

Materials and Methods: We generated pro-oligogenic NPCs (proOPCs), and neurogenic NPCs (NECs) from same line of hiPSCs. To generate hiPSCs, we made use of the non-viral piggyBac transposon system. We compared the regenerative therapeutic capacity of these oligogenic vs. neurogenic NPCs derived from hiPSCs in Adult Rowett Nude rats following bilateral C6-level clip contusion- compression injury.

Results: Eight weeks after transplantation, grafted hiPSC derived pro-OPCs and NECs survived and migrated within the injured spinal cord, and differentiated into the three major neural lineages in differing proportions. pro-OPCs successfully demonstrated an in vivo propensity to form cells from the oligodendroglial lineage (APC + and Olig2 +; $53.2 \%$ ), while NECs were significantly more likely to differentiate into cells with a neuronal profile (NeuN + and TuJ1 +; 52.01\%) (Figure 1). Both hiPSC-NECs and hiPSC-pro-OPCs contributed to tissue sparing and reduction in cavity size (up to $65 \%$ reduction in cell transplanted groups compared to vehicle) and promoted the survival of endogenous NeuN positive neurons as well as ChAT positive neurons. Functional recovery was observed in the forelimb grip strength (Figure 2) and gait locomotor function by using CatWalk at 10 weeks after injury with both cell types, but the effect of hiPSC-NECs was more pronounced in walking speed and stride length. In addition, although increased neuropathic pain after cell-based treatment is a potential concern, no increase in thermal and mechanical allodynia was observed in either group as assessed by tail flick and von Frey test, respectively. Furthermore, we evaluated long-term safety of these cell types up to 140 days after transplantation in NOD / SCID mice. The transplanted cells survived, but did not result in any microscopic tumors.

Conclusions: Both cell transplantation therapies using pro-OPCs and NECs derived from hiPSCs induce functionally significant repair and regeneration of the injured cervical spinal cord. pro-OPCs contributed to remyelination while NECs contributed to the rebuilding of neuronal circuits following SCI. These results move stem cell therapies closer to clinical translation.

Presentation \#48. Time-Dependent Vascular Remodeling and Inflammation following Decompression in Cervical Myelopathy

Wenru Yu, MD, North York, ON, Canada; Anna Badner, Toronto, ON, Canada; Michael G. Fehlings, MD, PhD, Toronto, ON, Canada

Introduction: Cervical spondylotic myelopathy (CSM), the most common cause of spinal cord impairment worldwide, results from progressive spinal cord compression by the degenerating cervical spine. Although CSM is currently treated with decompression, little is known of the vascular and inflammatory response under chronic compression and the subsequent decompression of the cervical spinal cord. Here, we aimed to examine the role of inflammation and vascular remodeling in a mouse model of progressive compression and decompression. Moreover, using human neuropathological samples, we demonstrate the clinical relevance of our CSM mouse model and the complex processes involved in inflammation and ischemia.

Methods: A synthetic polyether material was implanted under the C6 lamina of $\mathrm{C} 57 \mathrm{~B} / \mathrm{L}$ mice for 6 or 16 weeks, to model moderate and severe CSM respectively. The animals were subsequently followed for short-term (4 weeks) and long-term (3 months) periods after surgical decompression. Neurobehavioral outcomes were measured using the CatWalk system and rotarod test. We also investigated in detail the inflammatory response and blood vessel changes by immunohistochemistry, western blotting and Power Doppler readouts. The animal data was complemented by immunohistochemistry results from human post-mortem spinal cord tissue from individuals with CSM.

Results: When we compared human CSM tissue with that of controls, we found significant up- regulation of the inflammatory response, including HLA-DR Antigen (human leukocyte antigen), Iba1 and CD68 positive macroglia / macrophages, oxidative injury (P22-phox and INOS), reduced anti- inflammatory IL-10 positive cells and increased anti-inflammatory M2 positive cells. We also found a significant increase in the density of blood vessels and an increase in expression of vessel wall markers (fibronectin, PDGFR-B, Von Willebrand Factor) at the compression epicenter of human CSM cases. Next, using CSM mice, we found flattening of the spinal cord, neuronal loss, inflammation and gliosis with reduced blood flow to the spinal cord and increased vascular density at the compressed epicenter in moderate and severe CSM mice. In moderate CSM followed by a short-term (4 week) period post- decompression, we found an increased inflammatory response (Iba1, glectin-3 and GFAP expression) and vessels reperfusion compared to the CSM at 10 weeks. Interestingly, in severe CSM followed by a long-term ( 3 month) period post-decompression, the mice had significantly increased blood flow support to spinal cord as measured by Power Doppler, a reduced inflammatory response (Iba1, glectin-3 and GFAP expression), and increased number of neurons as well as vessels. Moreover, these animals had an increased spinal cord size and improve functional recovery, determined by the rotarod test.

Conclusion: We report novel evidence that inflammation and 
ischemia are critical to inducing neural degeneration in the setting of progressive CSM. As decompression induces early ischemia and an inflammatory response, mice require a longer period of time (2 to 3 months) to recover their spinal cord size and functional vasculature. Taken together, this supports the clinical results that demonstrate decompression is beneficial in CSM.

\section{Presentation \#49. Are Patient Reported Outcomes Predictive of Patient Satisfaction Five Years after Anterior Cervical Spine Surgery?}

Gregory D. Schroeder, MD, Philadelphia, PA; Han Jo Kim, MD, New York, NY; Todd J. Albert, MD, New York, NY; Kris E. Radcliff, MD, Egg Harbor Township, $N J$

Introduction: Patient satisfaction is becoming an increasing common proxy for surgical quality; however, the correlation between patient satisfaction and surgical outcomes two and five years after anterior cervical surgery has not been evaluated. The purpose of this study is to determine if patient satisfaction is predicted by improvement in patient reported outcomes (PRO) five years after anterior cervical spine surgery.

Methods: A retrospective analysis of prospectively collected data was reviewed. Patients were randomized $(2: 1)$ to TDR or ACDF at one or two contiguous levels from $\mathrm{C} 3$ to $\mathrm{C} 7$ as part of an FDA IDE trial. For the present analysis, patients in both surgical groups were combined. Patients were assessed for satisfaction, NDI, VAS neck pain, and SF-12PCS / MCS scores at 24 and 60 months. Receiveroperating characteristic (ROC) curves were utilized to determine if improvement in different PRO metrics can accurately identify patient satisfaction.

Results: Data was available for 512 patients at 60 months with 437 patients as "very satisfied", 50 patients as "somewhat satisfied", 16 patients as "somewhat dissatisfied", and 9 patients as "very dissatisfied." There was no significant difference in the number of one- or two-level patients in each satisfaction classification $(p=0.17)$, and no difference was found among 60-month satisfaction and age ( $\mathrm{p}$ $=0.73)$, gender $(p=0.49)$, race $(p=0.06)$, or BMI $(p=0.69)$. Baseline SF-12 PCS scores were significantly different among very satisfied (33.5), somewhat satisfied (32.2), somewhat dissatisfied (35.8), and very dissatisfied $(25.9)$ groups $(p=0.0023)$ at 60 months. When baseline outcomes scores were compared across 60-month satisfaction groups, no difference was seen among baseline NDI, VAS neck pain or SF-12 MCS scores. A significant difference was found across baseline SF-12 PCS scores ( $\mathrm{p}<0.0001)$ (Table 1).

Patient satisfaction was significantly associated with patient outcomes at 60 months. NDI, VAS neck pain, and SF-12 MCS / PCS scores and improvement from baseline were significantly different between satisfaction classifications at 60 months $(\mathrm{p}<0.001$; Table 1). Mean NDI was $15.1 \pm 16.0$ for the very satisfied, $36.6 \pm 17.5$ for somewhat satisfied, $38.3 \pm 18.9$ for somewhat dissatisfied, and 57.6 \pm 21.4 for very dissatisfied patients. Mean VAS neck pain was 15.2 \pm 22.7 for very satisfied, $47.1 \pm 29.6$ for somewhat satisfied, $59.1 \pm$ 30.5 for somewhat dissatisfied, and $64.7 \pm 29.6$ for very dissatisfied patients. The mean SF-12 MCS score was $52.2 \pm 9.6,45.7 \pm 12.6$, $46.6 \pm 14.2$ and $38.6 \pm 12.1$ for the very satisfied, somewhat satisfied, somewhat dissatisfied and very dissatisfied patients, respectively. Similarly, the mean SF-12 PCS score was $48.2 \pm 10.6,37.5 \pm 9.2$, $34.3 \pm 5.5$ and $29.7 \pm 8.5$ for the very satisfied, somewhat satisfied, somewhat dissatisfied and very dissatisfied patients, respectively.

The results of the ROC analysis at five years demonstrated that improvements in NDI and VAS, and the absolute NDI and VAS scores at 60 months had excellent accuracy at differentiating patient satisfaction (AUC $=0.80-0.86$ ). Comparatively both the improvement in, and the absolute value of the SF-12 PCS had poor accuracy at differentiating patient satisfaction $(\mathrm{AUC}=0.64-0.68$; Figure 1).
Conclusion: In patients undergoing one and two level anterior cervical spine surgery, at two and five years postoperatively patient satisfaction is significantly predicted by patient reported outcomes including the VAS neck score and the neck disability index.

\section{Presentation \#50. Diminished Mental Health Prior to Cervical Fusion Can Have a Profound Effect on Patient Derived Outcomes Depending on Presenting Diagnosis: Results of a Prospective Surgeon Driven Cervical Database at 2 Years}

Peter G. Passias, MD, Westbury, NY; Cyrus M. Jalai, BA, New York, NY; Bassel G. Diebo, MD, Long Island City, NY; Michael C. Gerling, MD, New York, NY; Gregory W. Poorman, BA, New York, NY; Kris E. Radcliff, MD, Egg Harbor Township, NJ; Paul Arnold, MD, FACS, Kansas City, KS; Jeffrey A. Rihn, MD, Media, PA; Eli Baron, MD, Los Angeles, CA; Robert E. Isaacs, MD, Durham, NC; Paul A. Anderson, MD, Madison, WI; Alexander Vaccaro, MD, PhD, Gladwyne, PA

Introduction: Optimizing outcomes is essential for effective surgical treatment of cervical spine disorders. For cervical spine pathologies, improvements in neck disability and physical functioning are important indicators of surgical success. Though mental impairment is commonly noted among patients with cervical spine complaints, comparative studies on baseline mental status and overall patientreported improvement in specific diagnoses have not been proposed. This study analyzes patient reported outcomes over 2-years postoperative among cervical myelopathy and cervical radiculopathy diagnoses dependent on pre-operative mental status.

Materials and Methods: This was a retrospective analysis of a database of patients with cervical pathology prospectively collected from a multicenter spine registry. Inclusion criteria were patients diagnosed with either cervical spondylosis with myelopathy ('MYELO') or radiculopathy ('RADIC': cervical disc herniation, cervical stenosis, cervical spondylosis without myelopathy) and with complete follow-up through 2-years post-operative. Patients were assessed for the following health-related quality of life (HRQL) measures at baseline and 6-, 12-, and 24-months post-operative: Neck Disability Index (NDI), Short Form-36 (SF) Physical (PCS) and Mental (MCS) Component Summaries. Baseline MCS score for all included patients were dichotomized using 60th (MCS-HI) vs. 40th percentiles (MCS-LO), and in each diagnoses MCS groups were propensity score matched for baseline NDI value. Independent and paired t-tests compared improvement in each patient diagnosis group for MCS-HI and MCS-LO cohorts.

Results: A total of 439 patients were included for analysis (mean age $53.9 \pm 10.5$ years; mean BMI

$28.6 \pm 5.6 \mathrm{~kg} / \mathrm{m} 2 ; 62.9 \%$ female). The mean baseline MCS score was $39.1 \pm 14.2$, and was dichotomized into lower-40th (MCS-LO $\leq 39.00$; mean $26.7 \pm 7.2$ ) and upper-60th (MCS-HI $\geq 42.00$; mean $51.5 \pm 6.7)$ percentile groups based on the score distribution for the total cohort. For MYELO, propensity matching gave 34 patients in each MCS group. At baseline, MYELO patients in both MCS-HI and MCS-LO had statistically similar NDI and PCS scores $(\mathrm{p}>0.05)$. However at 2-year follow-up, MYELO MCS-LO had significantly worse NDI (17.8 vs. 8.7, p < 0.001), despite overall baseline-2-year improvement in NDI $(p=0.003)$. Myelopathy patients in the MCSHI group displayed significant overall improvement in both PCS and NDI by 2 -years post-operative $(\mathrm{p}<0.015)$. Following propensity matching for radiculopathy patients, $\mathrm{N}=52$ were in each MCS group. At baseline and 2-year post-operative, RADIC MCS-HI and MCSLO were statistically similar for both NDI PCS $(p>0.05)$. However, both MCS-HI and MCS-LO radiculopathy patients demonstrated significant improvement in overall PCS and NDI scores with treatment $(\mathrm{p}<0.015)$.

Conclusions: This study revealed that pre-operative mental status, gauged by the SF-36 Mental Component Score, may be a useful tool in identifying discrepancies in overall patient-reported outcomes 
depending on the specific cervical spine diagnosis. Additional screening and care should be implemented for patient with cervical myelopathy for optimization of functional outcomes and disability status following surgical intervention.

\section{Presentation \#51. Which Domains of the NDI Improve Most after Surgery for Cervical Myelopathy?}

Paul W. Millhouse, MD, MBA, Philadelphia, PA; Kristen Nicholson, PhD, Philadelphia, PA; Emily Pflug, BS, Philadelphia, PA; Barrett I. Woods, MD, Galloway, NJ; Gregory D. Schroeder, MD, Philadelphia, $P A ; D$. Greg Anderson, MD, Moorestown, NJ; Christopher K. Kepler, MD, MBA, Philadelphia, PA; Mark F. Kurd, MD, Bryn Mawr, PA; Jeffrey A. Rihn, MD, Media, PA; Alexander R. Vaccaro, MD, PhD, MBA, Gladwyne, PA; Alan S. Hilibrand, MD, Philadelphia, PA; Kristen E. Radcliff, MD, Egg Harbor Township, NJ

Introduction: The neck disability index (NDI) is an easily scored, common, ten-item questionnaire about symptoms relevant to cervical spine pathology: pain intensity, personal care, lifting, reading, headaches, concentration, work, driving, sleeping, and recreation. Initial validation for the NDI considered only "whiplash"-injury patients in an outpatient clinic and was published in the physical therapy literature. However, the NDI is now widely used to evaluate the outcomes of cervical surgery. The purpose of this study was to determine which domains of the NDI improve most after cervical spine surgery for myelopathy and whether improvement in the composite NDI score or specific domains better predicts change in physical function.

Materials and Methods: Analysis of a prospectively-kept registry of patients treated at a major academic medical center. At baseline standardized outcome measures including NDI and SF12 PCS were collected. Preoperative outcome measures were compared to those at one year after surgery using paired Student's t-tests. For this study, each of the ten items was treated separately. Multiple linear regressions were performed using change in SF12 PCS as the dependent variable and change in NDI components as the independent variables.

Results: Baseline data were collected on 118 patients (mean age 58 years). A total of 66 patients had complete 1 year follow-up data. Each of the ten NDI components significantly improved from baseline $(\mathrm{p}<0.004)$. The NDI items with the largest improvements from baseline were: sleeping (-1.5 mean change from baseline), recreation (-1.443), lifting (-1.186), work (-1.043), and pain (-1.014). Linear regression for change in NDI components versus change in SF12 PCS revealed a significant correlation $(\mathrm{r} 2=0.407, \mathrm{p}<0.001)$. The only significant $(\mathrm{p}=0.001)$ predictor value was change in recreation score $(-2.41,95 \%$ CI $-3.81,-1.00)$. "Lifting" was the only other factor with a robust coefficient $(-1.21,95 \% \mathrm{CI}-2.42,0.00)$ although this was not significant $(p=0.051)$. Linear regression for change in the composite NDI and change in PCS was significant $(\mathrm{p}<0.001)$, and had a weaker correlation $(\mathrm{r} 2=0.315)$. A linear regression incorporating only "recreation" and "lifting" had an r-squared value of $0.434(p<0.001)$.

Conclusion: All domains of the NDI do not improve equally after surgery for myelopathy. The pain subdomain had only a moderate observed improvement and a poor correlation to health related quality of life. Some specific domains correlate more strongly with improvement in health related quality of life than the composite NDI score. Based upon these results, we conclude that the item bank and composite scoring of the NDI are inappropriate for evaluating quality of life in studies of surgically treated cervical spondylotic myelopathy patients.
Presentation \#52. Concurrent Validity and Responsiveness of PROMIS Health Related Quality of Life Assessment in Patients with Cervical Spine Disease

Richard L. Skolasky Jr., ScD, Baltimore, MD; Shalini Selvarajah, MD, MPH, Baltimore, MD; Brian J. Neuman, MD, Baltimore, MD

Introduction: The ability to validly and reliably measure patientreported outcomes (PRO) is an important undertaking to ensure that we can capture how effectively our treatments are affecting our patients' wellbeing. A potential tool to demonstrate quality of care and minimum clinically important differences (MCID) following surgery is the NIH-funded PROMIS instrument, which has been psychometrically validated to measure PROs among research participants with various chronic diseases and demographic characteristics. We sought to demonstrate the concurrent validity and responsiveness of the PROMIS instrument among individuals with cervical spine disease who had undergone surgical intervention.

Materials and Methods: This was a single-institution prospective observational cohort study of all patients with spine diseases who had received surgical intervention. Fifty consecutive patients who underwent surgery for cervical spine disease were included in this analysis. Seven PROMIS health domains (pain intensity, physical function, fatigue, depression, anxiety, sleep disturbance, and satisfaction with social roles) and four legacy measures (SF12, NDI, pain intensity and interference) were collected pre-operatively, and at least once within six months post-operatively. Concurrent validity was demonstrated using correlation coefficients of PROMIS health domains with legacy measures at the pre-operative assessment. Responsiveness of PROMIS health domains was determined using the methods of Coyne, et al. (Qual Life Res, 2005). MCIDs of PROMIS health domains were determined using the change in pre-operative to post-operative (within 6 months) scores anchored to the NASS Patient Satisfaction Index (PSI) using the methods of Eton, et al. (J Clin Epi, 2004).

Results: All seven PROMIS domains showed moderate to strong correlations with NDI, MCS intensity of neck pain and pain interference while they were generally weakly correlated with the intensity of arm pain (Table 1).

PROMIS domains were generally well correlated with PCS with the exception of physical function which showed a weak negative correlation. The PROMIS pain domain demonstrated large responsiveness (-.92), while anxiety (-.63), sleep disturbance (-.58) and social role $(.59)$ were associated with moderate responsiveness. Physical function (.37), fatigue (-.33) and depression (-.44) had small responsiveness. Using the anchor-based method, the MCIDs for each PROMIS domain were: -4.27 for pain, +3.18 for physical function, +.16 for fatigue, -3.9 for anxiety, -3.33 for depression, -1.94 for sleep disturbance and +.93 for social role, although statistical significance was only reached for pain.

Conclusions: PROMIS health domains can be used to validly assess post-operative patient-reported wellbeing in this. PROMIS health domains were responsive to reductions in symptoms and improvements in quality of life after surgery.

Presentation \#53. A Comparison of Patient Centered Outcome Measures to Evaluate Dysphagia and Dysphonia after Anterior Cervical Discectomy and Fusion (ACDF)

Alpesh A. Patel, MD, River Forest, IL; Surabhi Bhatt, BS, Chicago, $I L$; Junyoung Ahn, BS, Chicago, IL; Jason W. Savage MD, Chicago, IL; Wellington K. Hsu MD, Chicago, IL; Kern Singh, MD, Chicago, IL

Introduction: Dysphagia and dysphonia are common complications after anterior cervical spine surgery. Despite their clinical importance, studies on the treatment and / or prevention of these complications are limited by the lack of valid and reliable outcome measures. Two 
new patient-centered outcome measures - the Eating Assessment Tool (EAT-10) Voice Handicap Index (VHI-10) - have been shown to have excellent validity and reliability in the otolaryngology patient population. These instruments may be used to document the dysphagia or dysphonia severity and monitor treatment response in patients with swallowing and voice disorders after anterior cervical spine surgery.

Materials and Methods: Following internal IRB approval, patients undergoing 1 to 3-level ACDF were recruited from two tertiary spine centers. Each patient prospectively complete the eating assessment tool (EAT-10), the voice handicap index (VHI-10), and the Bazaz score questionnaire prior to surgery, 1 day, 2 weeks, 6 weeks, 12 weeks, 6 months, and 1 year post-operatively. Mean scores were compared through ANOVA and proportion of patients with clinically significant scores (EAT-10 $\geq 3$ and VHI-10 > 11) among each follow-up time. Internal reliability of EAT-10 and VHI-10 was tested via Cronbach's while Pearson's correlation testing was employed to assess the correlation of EAT-10 to the Bazaz score.

Results: A total of 100 patients were included in the study, from which 85 completed 6 month follow-up and 64 completed 1 year follow-up. Baseline mean NDI scores were $18.74(+/$ - 8.66) and improved to $7.52(+/-7.27)$ at 6 months $(\mathrm{p}<0.05)$ and $8.22(+/$ - 7.65) at 12 months $(\mathrm{p}<0.05)$. Baseline EQ-5D index scores were $0.59(+/-0.23)$ and improved to $0.79(+/-0.16)$ at 6 months $(\mathrm{p}<$ $0.05)$ and $0.75(+/-0.17)$ at 12 months $(\mathrm{p}<0.05)$. EAT-10 $(=0.978)$ and VHI-10 $(=0.900)$ demonstrated excellent internal reliability, in addition to the EAT-10 showing significant correlation to Bazaz $(r=$ 0.794 ) across all time points. Mean EAT-10 and VHI-10 scores were significantly highest at 1 day post-op $(p<0.05)$. While mean EAT-10 scores increased with severity of dysphagia as defined by the Bazaz score, 10 of 556 scores $(1.8 \%)$ of patients that claimed "no dysphagia" or "mild dysphagia" by the Bazaz score had clinically significant dysphagia (EAT-10 $\geq 3$ ).

Conclusions: The EAT-10 and the VHI-10 scores showed excellent internal reliability. In addition, the EAT-10 score was an accurate measure across mild to severe dysphagia, and captured significant dysphagia in patients that would have otherwise been missed using the Bazaz score. The EAT-10 and VHI-10 surveys can provide a better measure of postoperative dysphagia and dysphonia than current outcomes used in spine surgery.

\section{Presentation \#54. The Seven-Year Cost-Effectiveness of Anterior Cervical Discectomy and Fusion vs. Cervical Disc Arthroplasty}

Steven J. McAnany, MD, New York, NY; Samuel C. Overley, MD, New York, NY; Jun Sup Kim, MD, New York, NY; Robert Brochin, MD, New York, NY; Sheeraz A. Qureshi, MD, MBA, New York, NY

Introduction: Anterior cervical discectomy and fusion (ACDF) and cervical disc replacement (CDR) are both acceptable surgical options for the treatment of cervical myelopathy and / or radiculopathy. Studies have demonstrated non-inferiority of CDR when compared with ACDF in large randomized investigational device exemption (IDE) studies. Furthermore, economic analysis of the two procedures at two years has demonstrated that CDR may be the cost-effective treatment option. The purpose of this study is to determine the seven year cost-effectiveness of single-level ACDF versus CDR.

Materials and Methods: A Markov-state transition model (Figure 1) was used to evaluate data from the Prestige Cervical Disc IDE study. Data from the 36-item Short Form Health Survey were converted into utilities using the SF-6D algorithm for 212 CDR patients and 183 ACDF patients. Costs were calculated from the payer perspective using a 140\% multiple of 2014 Medicare reimbursement for diagnosis related groups (DRG) and current procedural terminology (CPT) codes. Transition probabilities in the model were determined from complication rates as well as index / adjacent segment re-operation rates from the IDE study. Quality adjusted life years (QALY's) were used to represent effectiveness. For the base case analysis, incremental cost effectiveness ratios (ICER's) were used to compare treatments. A willingness-to-pay threshold of \$50,000 / QALY was used. A probabilistic sensitivity analysis was performed using a Monte Carlo simulation of 10,000 cycles to validate the input variables in the model. Confidence intervals (CI) were reported at $95 \%$.

Results: The base case assumed an ideal operative candidate of 40 years old who has failed appropriate conservative care. The base case analysis generated a seven year cost of $\$ 172,989$ for CDR and $\$ 143,714$ for ACDF. CDR resulted in a generation of 4.52 QALY's while ACDF resulted in 3.85 QALY's. The ICER was calculated to be $\$ 43,522$ / QALY for CDR which was less than the \$50,000 / QALY WTP threshold. CDR and ACDF were both cost-effective procedures (\$38,247 / QALY vs. \$37,325 / QALY). The Monte Carlo simulation validated the base case scenario (Table 2). CDR had an average cost of

$\$ 173,190$ (CI: $\$ 144,353 ; \$ 202,479)$ with an average effectiveness of 4.52 (CI: $3.99 ; 5.01$ ). ACDF had an average cost of $\$ 143,806$ (CI: 120,668; \$166,742) and an average effectiveness of 3.85 (CI: $3.39 ; 4.29)$. The ICER was calculated at $\$ 43,937$ / QALY in favor of CDR. Assuming a WTP \$50,000 / QALY, the cost-effectiveness acceptability curve indicated that CDR would be chosen $56 \%$ of the time based on 10,000 simulations (Figure 2 and 3).

Conclusions: CDR and ACDF are both cost-effective strategies at seven years. Based on the results of this model, CDR was found to be to the more cost-effective option with an ICER less than the

$\$ 50,000$ / QALY WTP threshold. Furthermore, the assumptions used in the base case analysis were strongly validated with the results of the probabilistic sensitivity analysis. Additional long-term studies (10 years) evaluating the clinical and quality-of-life outcomes of these two strategies are needed to further validate the findings in this model.

\section{Presentation \#55. Progressive Bone Formation after Cervical Replacement: Minimum of 5-Year Follow-up}

Feifei Zhou, MD, Beijing, China; Kevin L. Ju, MD, Atlanta, GA; John G. Heller, MD, Atlanta, GA; Yu Sun, MD, Beijing, China

Background: Cervical Disc Replacement(CDR) can be complicated by postoperative ossification and unwanted ankylosis at the index level, which some authors have termed "heterotopic ossification". However, this terminology may be inaccurate as it assumes the postoperative bone formation is unnatural and a consequence of the CDR surgery. We advocate describing this phenomenon as one of progressive bone formation to reflect the fact that it has more to do with individual patient factors rather than the CDR surgery. The objective of our study was to examine the prevalence, clinical significance, ramifications, and possible etiology of postoperative bone formation at the index level after CDR with a minimum of 5 years of follow-up data.

Methods: A retrospective review was performed on 61 patients (76 levels) who underwent Bryan disc replacements by a single group within one institution between 12 / 2003 and 8 / 2008. All patients had at least 5 years of clinical follow-up. Postoperative bone formation at the index level was graded on lateral cervical spine radiographs using the McAfee classification. Patients were divided into two groups, those with and without postoperative bone formation. Clinical outcomes such as Japanese Orthopaedic Association (JOA) score for cervical spondylotic myelopathy (CSM) and Neck Disability Index (NDI) and Visual Analogue Scale (VAS) for neck pain and arm pain in cervical spondylotic radiculopathy (CSR) patients were collected pre- and post-operatively and then compared between groups. The radiographic parameters analyzed included: the degree of preoperative cervical spondylosis on cervical radiographs utilizing the Kellgren classification, segmental range of motion (ROM) on lateral flexionextension radiographs, and the incidence of postoperative adjacent segment degeneration.

Results: The study patients had an average age of 43 years, with a mean follow-up of 94.2 months. The overall incidence of postoperative bone formation was 50\% (38/ 76 levels). Clinical outcomes in CDR 
patients at final follow-up were significantly improved for CSM and CSR patients by all four patient-reported measures $(\mathrm{p}<0.001)$. Despite decreased ROM $(\mathrm{p}<0.001)$, patients with postoperative bone formation had no significant differences in any of the four measures compared to those without postoperative ossification. Notably, patients with more severe preoperative cervical spondylosis at the surgical level had higher rates of postoperative bone formation at final follow-up ( $p=$ 0.036). Similarly, more severe preoperative spondylosis also correlated with higher rates of adjacent segment degeneration (ASD) $(p=0.010)$. Patients with postoperative ossification had higher rates of ASD ( $p$ $=0.007$ ), but there was no correlation between the severity of bone formation around the CDR and the incidence of ASD.

Conclusion: The overall incidence of postoperative bone formation after CDR was relatively high when patients are followed for greater than 5 years. However, this did not adversely affect patient reported outcomes even if it did decrease segmental ROM. Most notably, patients with more severe preoperative cervical spondylosis had higher rates of postoperative ossification. This suggests that postoperative ossification at the CDR segment is likely one of progressive bone formation in individuals already predisposed to forming bone rather than one of alleged heterotopic ossification as a consequence of the surgery, highlighting the importance of proper patient selection.

\section{Presentation \#56. Unintended Fusion in Cervical Artificial Disc Replacement: A Prospective Study on Heterotopic Ossification with 5 Years Follow-up}

Catarina Marques, MD, Uppsala, Sweden; Anna MacDowall, MD, Uppsala, Sweden; Martin Skeppholm, MD, PhD, Lowenstromska Sjukuset, Sweden; Nuno Canto Moreira, MD, PhD, Uppsala, Sweden; Claes Olerud, MD, PhD, Uppsala, Sweden

Introduction: Anterior cervical discectomy and fusion (ACDF) is the gold standard for treatment of cervical radiculopathy. However, concerns about the decrease in motion and its potential to affect on the adjacent segments lead to the development of motion-preserving implants for artificial disc replacement (ADR), as an alternative to ACDF. By preserving segmental motion, ADR is expected to decrease the incidence of adjacent-level degeneration associated to fusion, leading to better clinical outcomes. Heterotopic ossification (HO) is a complication of ADR that may lead to fusion, thus failing to preserve motion. Its real incidence in the cervical spine segment is still under debate, with only a few studies reaching long-term follow-up. Our aim was to evaluate $\mathrm{HO}$ rates after cervical ADR over a 5-year period, and to analyse factors that could contribute for this complication.

Material and Methods: This study is a post-hoc from a multicentre prospective randomised study in which 151 patients were blindly randomised to receive $\mathrm{ADR}$ or $\mathrm{ACDF}$, at a maximum of two cervical levels. The 81 patients that received ADR were treated postoperatively with NSAID for 10 days. Of those, 42 patients (57 prosthesis) who had good-quality radiological follow-up studies both at 2 years and at 5 years were included. 33 patients were women and 24 men, aged 35 to 59. Plain radiographs at 2 and 5 years after surgery where read by two experienced viewers, and graded in 5 levels according to a modified McAfee classification. Pre-operative degeneration on MRI, number and location of operated levels were assessed in order to establish factors that can influence the appearance and severity of $\mathrm{HO}$.

Results: HO was found in $91,2 \%$ and $82,4 \%$ of prosthesis at 5 years and 2 years follow-up respectively. Severe HO (grade 3 or 4 ) was found in $66,7 \%$ and complete fusion (grade 4 ) in $24,6 \%$ at 5 years. 36 prosthesis $(63,2 \%)$ did not increase the severity of $\mathrm{HO}$ between the 2- and 5-year follow-up. All the 5 that changed more than one grade $(8,8 \%)$ over this period were female. Women had a statistically significant $(\mathrm{p}<0,005)$ lower amount as well as lower grades of $\mathrm{HO}$ at all times. Age of the patients, place / amount of operated levels, or the severity of preoperative degeneration at adjacent levels on MRI did not influence the appearance of $\mathrm{HO}$.
Conclusion: Cervical ADR is meant to move. Our results show an ossification rate of $91,2 \%$ and a fusion rate of $24,6 \%$ despite the fact that the patients where given prophylactic NSAID to prevent them. Although $\mathrm{HO}$ grade slightly increases with time, it occurs much more often early in follow-up. Female gender is clearly a protective factor from $\mathrm{HO}$ in cervical ADR. No difference in preoperative degeneration grade was found that could explain the difference in incidence between men and women. Further analysis is needed in order to establish if and how unintended fusion in cervical ADR affects clinical outcomes.

Presentation \#57. Clinical Implications of Heterotopic Ossification after Cervical Disc Arthroplasty at 7 Years

Pierce D. Nunley, MD, Shreveport, LA; Eubulus J. Kerr, MD, Shreveport, LA; David A. Cavanaugh, MD, Shreveport, LA; Andrew Utter, MD, Shreveport, LA; Kelly Frank, MS, Shreveport, LA; Marcus B. Stone, PhD, Shreveport, LA

Introduction: Treatment with cervical disc arthroplasty (CDA) has been studied in multiple clinical trials meeting Level 1 evidence. Long-term data revealed that development of heterotopic ossification (HO) is possible, but the mechanism and clinical impact is unknown.

Materials and Methods: A total of 389 patients were treated with 1 or 2-level CDA for a prospective, randomized, FDA clinical trial across 24 sites in the US with 7-year follow-up. Patient characteristics including age, sex, BMI and type of work were collected preoperatively. Clinical and radiographic outcomes included $\mathrm{HO}$, flexion / extension range of motion (ROM), NDI score, VAS neck pain score, and patient satisfaction. All radiographic evaluations were conducted by independent radiologists (MMI Inc., Houston, TX). HO was classified using the system adapted from McAfee and Mehren. HO grades 3 and 4 were classified as clinically relevant due to restricted ROM. Fisher's exact test $(\mathrm{P}<0.05)$ was used to test significant differences across groups.

Results: Grade 0 / 1 HO was present in $4.6 \%$ of patients, grade 2 in $67.0 \%$, grade 3 in $17.4 \%$, and grade 4 in $11.0 \%$. Clinically relevant $\mathrm{HO}$ was observed in $28.4 \%$ of 1 -level patients. Between 5 and 7 years, $5.8 \%$ of patients had progression of HO 1 or 2 grades, with $94.2 \%$ showing no progression. Obese patients (preop BMI $\geq 30$ ) had a higher prevalence of clinically relevant $\mathrm{HO}$ than non-obese patients $(43.8 \%$ vs. $22.1 \% ; \mathrm{p}=0.035)$, and males had a higher prevalence than females $(37.3 \%$ vs. $20.7 \%$; $=0.088)$. Clinically relevant $\mathrm{HO}$ was present in $32.6 \%$ of 2-level patients. At the superior level, grade $0 / 1$ was present in $4.5 \%$ of patients, grade 2 in $73.2 \%$, grade 3 in $15.9 \%$, and grade 4 in $6.4 \%$. At the inferior level, grade $0 / 1$ was present in $3.4 \%$ of patients, grade 2 in $65.8 \%$, grade 3 in $26.2 \%$, and grade 4 in $4.7 \%$. From 5 to 7 years, $14 \%$ of patients showed HO progression of 1 or 2 grades, the remaining $86 \%$ of patients had no progression of $\mathrm{HO}$. In 2-level CDA, clinically relevant $\mathrm{HO}$ was more prevalent in males vs. females $(53.5 \%$ vs. $25.0 \%, \mathrm{p}<0.001)$, and a higher prevalence in obese patients $(44.3 \%$ vs. $36.1 \% ; p=0.36)$. Patients with clinically relevant and non-clinically relevant HO had similar 7-year NDI and VAS neck pain scores. At 7 years, there were no cases of subsequent surgery because of $\mathrm{HO}$, and patients with clinically relevant $\mathrm{HO}$ were equally satisfied with their procedure.

Conclusion: At 7-year follow-up, the majority of patients (69.2\%) did not have clinically relevant HO. HO progression between 5 and 7 years was stable, $89.4 \%$ of patients had no further development of HO. Possible predictors for clinically relevant $\mathrm{HO}$ include preoperative obesity and male gender. Patients with clinically relevant $\mathrm{HO}$ maintained similar NDI and VAS neck pain scores to non-clinically relevant $\mathrm{HO}$ patients. 

Presentation \#59. Cervical Deformity Surgery does Not Result in
Post-operative Dysphagia: A Prospective Cohort Study

Sravisht Iyer, MD, New York, NY; Hongda Bao, MD, PhD, Nanjing, China; Han Jo Kim, MD, New York, NY; Justin S. Smith, MD, PhD, Charlottesville, VA; Michael P. Kelly, MD, Saint Louis, MO; Munish Gupta, MD, Saint Louis, MO; Todd J. Albert, MD, New York, NY; Themistocles S. Protopsaltis, MD, New York, NY; Gregory M. Mundis, MD, San Diego, CA; Peter Passias, MD, Westbury, NY; Brian Neuman, MD, Baltimore, MD; Eric Kleinberg, MD, Sacramento, CA; Virginie Lafage, PhD, New York, NY; Christopher P. Ames, MD, San Francisco, $C A$

Introduction: The majority of current work describing the incidence of dysphagia after cervical surgery has focused on degenerative disease such as cervical spondylotic myelopathy or radiculopathy. The incidence of dysphagia in patients undergoing surgery for cervical deformity has not previously been described in the literature. We attempt to address this deficiency by describing the rate of persistent dysphagia following surgery for cervical deformity. We hypothesized that patients undergoing surgery for cervical deformity would not have significant post-operative dysphagia at intermediate-term follow up.

Methods: This was a prospective cohort study seeking to enroll operative cervical deformity (CD) patients. The inclusion criteria were one or more of the following: cervical kyphosis $(\mathrm{CK})>10^{\circ}$, cervical scoliosis $(\mathrm{CS})>10^{\circ}, \mathrm{C} 2-7 \mathrm{SVA}>4 \mathrm{~cm}$ and / or chin-brow vertical angle $(\mathrm{CBVA})>25^{\circ}$. Demographic, operative and radiographic variables were recorded. Intermediate (3 month) and long-term (1 year) follow up was obtained. Dysphagia was recorded using the Quality of Life in Swallowing Disorders (SWAL-QOL) survey. Paired t-tests (continuous variables), Paired t-test, independent t-tests and bivariate Pearson correlations were performed.

Results: 88 patients were included in the study. The average age was $61.52 \pm 10.52$ years. Three month (intermediate) follow up was available for all patients $(100 \%), 45(51.1 \%)$ patients had 1 year SWAL-QOL scores for analysis. The mean pre-op SWAL-QOL was 78.35. There was no difference between pre-op and intermediate SWAL-QOL scores (78.4 vs. 77.3, $\mathrm{p}=0.527)$. SWAL-QOL scores improved in $54.5 \%$ of patients and worsened in $45.5 \%$ of patients.

Demographic Variables. Baseline SWAL-QOL was correlated with baseline NDI $(r=-0.49)$, mJOA $(r=0.39)$ and EQ5D $(r=$ -0.54). Increased Body Mass Index (BMI) was correlated worse baseline SWAL-QOL $(\mathrm{r}=-0.30)$. Patients who had had prior cervical surgery had a lower baseline SWAL-QOL score (74.3 vs. 82.0, P = 0.043). Age, gender, smoking and Charlson Comorbidity Index (CCI) showed no significant correlations at baseline. At 3 month follow up, a higher pre-operative CCI $(\mathrm{r}=-0.26)$ and a greater number of cervical procedures $(\mathrm{r}=-0.31)$ was correlated to worse SWAL-QOL score.

Radiographic Variables. There were no pre-operative variables that were correlated with the baseline total SWAL-QOL score or with 3-month total SWAL-QOL scores. Change in radiographic variables (i.e., deformity correction) was not correlated with change in total SWAL-QOL scores or 3-month total SWAL-QOL scores. Subgroup analysis of patients with pre-operative $\mathrm{CK}\left(\mathrm{CL}<0^{\circ}\right)$ and those without $\mathrm{CK}$. These groups had no difference in baseline, 3 month or change in SWAL-QOL scores. In the CK group, an increase in O-C2 angle was correlated with worse 3 month SWAL-QOL scores $(r=-0.37)$.

Surgical Variables. Number of levels fused, upper instrumented vertebrae (UIV), osteotomy use, surgical approach and steroid use had no effect on 3 month SWAL-QOL scores.

Conclusion: While the incidence of early dysphagia in pts undergoing $\mathrm{CD}$ surgery is unknown, we show that patients undergoing surgery for $\mathrm{CD}$ do not have dysphagia that persists at intermediate, 3 month follow up. Surgical techniques used to correct CD do not appear to have a significant impact on SWAL-QOL scores. Patients with prior cervical surgery and a higher BMI had lower baseline SWAL-QOL.
Presentation \#60. Pseudarthrosis in Patients Undergoing Multilevel Posterior Cervical or Cervical-Thoracic Fusions: Multi-Center Analysis

Eeric Truumees, MD, Austin, TX; Devender Singh, PhD, Austin, TX; Matthew J. Geck, MD, Austin, TX; John K. Stokes, MD, Austin, TX

Introduction: Pseudarthrosis after multilevel posterior cervical or cervical-thoracic fusions is a common complication. We investigated the effect of pseudarthrosis on cervical aligment. Futhermore, we report the effect of type of bone grafts on the rate of pseudarthrosis in patients undergoing multilevel posterior cervical or cervical-thoracic fusions.

Methods: We assembled a multicenter (4 sites) radiographic and clinical database of patients that had undergone 3 or more level posterior cervical or cervical-thoracic fusions for degenerative disease from January 2008 to May 2013 with at least 2 years of post-operative (post-op) follow-up. Patients were divided into two groups: group I (fusion ending in the cervical spine) and group II (fusion extending into the thoracic spine). All radiographic measurements were performed by an independent experienced clinical researcher. For the analysis, bone grafts were divided into four groups: local only; local and allografts; bone morphogenetic protein (BMP) only; and iliac crest only. Current smokers included those patients smoking at the time of or within 6 months of their surgery. Paired t-test was used to compare means. Analysis of variance (ANOVA) was used to investigate the effects of type of bone grafts on the rate of pseudarthrosis. Level of significance was set at $\alpha=0.05$.

Results: Rate of pseudarthrosis in group I and group II were $21.2 \%$ and $10.96 \%$, respectively. Mean age of patients with pseudarthrosis in group I and group II were 56( \pm 9$)$ and $67( \pm 4)$ years, respectively. Females had higher numbers of pseudarthrosis than males (group I: $67 \%$ vs. $33 \%$; group II: $55 \%$ vs. $45 \%$; p < 0.05 ). Overall, $53.3 \%$ of the patients with pseudarthrosis were current smokers. The rate of smoking in the solid fusion group was $21.9 \%$. The odds ratio of pseudarthrosis for a smoker compared with a non-smoker was 4.071 (95\% CI: $1.798-9.221)$. Mean number of spinal levels treated for patients with pseudarthrosis in group I and group II were 3.6( \pm $0.79)$ and 6.2 $( \pm 2.5)$, respectively. Mean T1 slope for patients with pseudarthrosis increased significantly ( 2 wk vs. 2 year post-op) in both groups $(\mathrm{p}<0.05)$. Both groups with pseudarthrosis had significantly higher mean $\mathrm{C} 2-\mathrm{C} 7$ sagittal plumbline at 2 years follow-up $(\mathrm{p}<0.05)$. Mean cervical lordosis decreased in both groups with pseudarthrosis (2 wk vs. 2 year post-op). The difference was not statistically significant $(p>0.05)$. Overall, ANOVA showed no significant effect of type of bone grafts on the rate of pseudarthrosis $(\mathrm{p}>0.05)$.

Conclusion: We conclude that pseudarthrosis affects cervical alignment in patients undergoing multilevel posterior cervical or cervical-thoracic fusions. The study did not find any significant effect of type of bone grafts on the rate of pseudarthrosis. Prospective studies with additional patients and greater statistical power are needed to further understand the implications of pseudarthrosis on cervical alignment.

Presentation \#61. Not All Patients with Diabetes have the Same Risk: The Association of Perioperative Glycemic Control with Deep Postoperative Infection following ACDF in Patients with Diabetes

Jourdan M. Cancienne, MD, Charlottesville, VA; Brian C. Werner, MD, Charlottesville, VA; Anuj Singla, MD, Charlottesville, VA; Hamid Hassanzadeh, MD, Charlottesville, VA; Francis H. Shen, MD, Charlottesville, VA; James A. Browne, MD, Charlottesville, VA; Adam L. Shimer, MD, Charlottesville, VA

Introduction: Diabetes mellitus has been associated with an increased risk for postoperative infection following spine surgery; 
however, among patients with diabetes, the level of perioperative glycemic control may affect the risk of postoperative infection. Patients with very tight perioperative glycemic control may have a lower risk of infection compared to patients with higher average glucoses in the perioperative period. The primary goal of the present study was to evaluate the association of perioperative glycemic control as demonstrated by hemoglobin a1c (HbA1c) in patients with diabetes with the incidence of deep postoperative infection following anterior cervical discectomy and fusion (ACDF) requiring operative irrigation and debridement. Our secondary objective was to calculate a threshold level of hemoglobin alc above which the risk of postoperative infection after ACDF increases significantly in patients with diabetes.

Methods: A national administrative database was queried for patients who underwent primary ACDF. Patients with diabetes mellitus who had a perioperative HbA1c level checked within 3 months of surgery were identified; and were then stratified into fourteen mutually exclusive groups based on their hemoglobin alc in $0.5 \mathrm{mg} / \mathrm{dl}$ increments from $<5.49 \mathrm{mg} / \mathrm{dl}$ to $>12 \mathrm{mg} / \mathrm{dl}$. The incidence of deep infection requiring operative intervention within 1 year for each $\mathrm{HbAlc}$ group was then identified using CPT and ICD-9 codes. A receiver operating characteristic (ROC) analysis was performed to determine an optimal threshold value of the HbAlc above which the risk of postoperative infection was significantly increased.

Results: 3,341 patients who underwent ACDF with diabetes and a perioperative $\mathrm{HbAlc}$ recorded within 3 months of surgery in the database were included in the study. The rate of deep infection requiring irrigation and debridement within one year postoperatively stratified by $\mathrm{HbA} 1 \mathrm{c}$ is pictured in Figure 1, which ranged from a low of $1.5 \%$ to a high of $6.4 \%$ and was significantly correlated with increasing $\mathrm{HbAlc}$ levels $(\mathrm{P}=0.001)$. The results of ROC analysis determined that the inflection point of the ROC curve corresponded to an $\mathrm{HbA1c}$ level above $7.5 \mathrm{mg} / \mathrm{dL}(\mathrm{p}=0.022, \mathrm{AUC}=0.67$, spec. $=68 \%$, sens $=46 \%)$.

Conclusions: The risk of deep postoperative infection requiring surgical intervention following ACDF in patients with diabetes mellitus increases as the perioperative $\mathrm{HbAlc}$ increases. ROC analysis determined that a perioperative HbA1c above $7.5 \mathrm{mg} / \mathrm{dL}$ could serve as a threshold for a significantly increased risk of deep postoperative infection following ACDF.

\section{Presentation \#62. Laminoplasty and Wide Decompression were Risk Factors of C5 Palsy: Analysis of 303 Surgical Cases with Cervical Compression Myelopathy}

Satoshi Nori, MD, PhD, Chiba, Japan Ryoma Aoyama, MD, PhD, Chiba, Japan Ken Ninomiya, MD, Chiba, Japan Junichi Yamane, MD, PhD, Tokyo, Japan Kazuya Kitamura, MD, PhD, Tokyo Japan Tateru Shiraishi, $M D, P h D$, Chiba, Japan

Introduction: C5 palsy is a potential complication after posterior cervical decompression. Before 2009, the incidence of C5 palsy was $9.5 \%$ (10 out of 105 patients) after double-door laminoplasty combined with laminectomy (DL) at our institute. Since 2009, we have performed laminectomy alone (LAM) of 2-3 $\mathrm{mm}$ wider than the spinal cord width which was measured on preoperative myelogram-CT. Incidence of C5 palsy was successfully reduced to $1.0 \%$ ( 2 out of 198 patients) after those changes. Purpose of this study is to elucidate the risk factors of C5 palsy by reviewing the surgical outcomes.

Materials and Methods: Out of 303 cervical myelopathic patients enrolled in this study, 105 patients underwent DL (cervical spondylotic myelopathy (CSM) 84 cases and ossification of the posterior longitudinal ligament (OPLL) 21 cases). The rest of 198 underwent LAM as wide as preoperatively planned (CSM 122 cases and OPLL 76 cases). We statistically analyzed risk factors of C5 palsy such as surgical procedures (DL or LAM), difference in width between surgical decompression and spinal cord (DW), dimension of C4 / 5 foramen measured at its narrowest point on axial CT, the amplitude of posterior spinal cord shift at C4 / 5 level (PSS) on sagittal MRI, post-operative $\mathrm{C} 2-\mathrm{C} 7$ angle on plain $\mathrm{X}$ rays, the number of consecutive levels decompressed, OPLL, T2 high intensity area at C3 / 4 level on MRI, age, gender, operation time and blood loss. Statistical analyses were performed using SPSS (version 22, SPSS, Inc.). Chi square test and one-way analysis of variance were used to compare the C5 palsy and no $\mathrm{C} 5$ palsy groups. Logistic regression analysis was used for risk factor analysis. First, the parameter significance was evaluated using univariate analysis. Factors with $\mathrm{p}<0.25$ in the univariate analysis were then induced in the multivariate analysis.

Results: There were significantly more patients with DL in C5 palsy group than no $\mathrm{C} 5$ palsy group $(66.7 \%$ and $11.0 \%, \mathrm{p}=1.67 \mathrm{E}-02)$. Significantly more males were observed in patients with C5 palsy than those without $(100 \%$ and $70.4 \%, \mathrm{p}=1.67 \mathrm{E}-02)$. DW $(10.4 \pm 4.5$ and $5.3 \pm 4.3, \mathrm{p}=5.16 \mathrm{E}-05)$, PSS $(2.1 \pm 1.1$ and $1.0 \pm 0.9, \mathrm{p}=7.38 \mathrm{E}-05)$, age $(70.3 \pm 6.9$ and $62.1 \pm 10.7, \mathrm{p}=9.80 \mathrm{E}-03)$, the numbers

of surgically interfered laminae $(3.4 \pm 0.9$ and $2.6 \pm 1.0, \mathrm{p}=$ 4.96E-03), operation time (181.1 \pm 56.7 and $141.2 \pm 40.4, \mathrm{p}=$ $1.13 \mathrm{E}-03)$, and blood loss $(94.0 \pm 96.4$ and $23.1 \pm 54.7, \mathrm{p}=3.02 \mathrm{E}-$ $05)$ were significantly greater in patients with $\mathrm{C} 5$ palsy than those without. Dimension of C4 / 5 foramen was significantly narrower in patients with C5 palsy than those without $(p=6.76 \mathrm{E}-04)$ (Table 1). Multivariate logistic regression analysis revealed that DL (OR, 17.2; 95\% CI, 2.8 to $103.9, \mathrm{p}=1.99 \mathrm{E}-03)$, DW (OR, 1.2; 95\% CI, 1.0004 to $1.4, \mathrm{p}=4.94 \mathrm{E}-02)$, dimension of $\mathrm{C} 4$ / 5 foramen $(\mathrm{OR}, 0.2 ; 95 \% \mathrm{CI}$, 0.08 to $0.6, \mathrm{p}=4.64 \mathrm{E}-03)$ and age (OR, $1.2 ; 95 \% \mathrm{CI}, 1.1$ to $1.4, \mathrm{p}=$ 5.14E-03) were considered as the risk factors of C5 palsy (Table 2).

Conclusion: Double-door laminoplasty, wide decompression, C4 / 5 foraminal stenosis and advanced age at surgery were considered as the risk factors of C5 palsy. Laminectomy of $2-3 \mathrm{~mm}$ wider than the spinal cord width dramatically reduced its incidence.

\section{Presentation \#63. Reoperation Rates following Open Door Cervical Laminoplasty}

John Rodriguez-Feo, MD, Charlotte, NC; Daniel Leas, MD, Huntersville, NC; Susan M. Odum, PhD, Charlotte, NC; Mark F. Kurd, MD, Bryn Mawr, PA; Bruce V. Darden II, MD, Charlotte, NC; R. Alden Milam IV, MD, Charlotte, $N C$

Introduction: Degenerative cervical myelopathy is a common cause of spinal cord dysfunction. Symptoms typically consist of upper and lower sensorimotor dysfunction. Surgical spinal cord decompression is effective in halting disease progression and allowing for neurological recovery. Instances of multilevel compression are commonly approached via a posterior approach with laminoplasty or laminectomy and fusion. Laminoplasty has been proven to be a safe and effective procedure that adequately decompresses the neural elements and allows for some motion preservation. The purpose of this study was to determine the reoperation rate after cervical laminoplasty and determine potential risk factors for reoperation.

Materials and Methods: We retrospectively reviewed our cohort who underwent open-door cervical laminoplasty between January 1, 2005 and October 31, 2012. Inclusion criteria included a minimum two-year follow-up or a reoperation. Follow up consisted of either a clinic visit or a telephone interview. Charts were reviewed with special attention to the age, sex, BMI, medical comorbidities including COPD and diabetes, workers compensation status, and duration of symptoms. Patient function was stratified according to the preoperative Nurick scale. Operative records were reviewed to determine the levels of laminoplasty performed, EBL, and if any concomitant procedures such as laminectomy, arthrodesis, and / or foraminotomies were performed. Postoperative notes were reviewed to determine if any complications requiring reoperations occurred as well as any $\mathrm{C} 5$ palsies. The reoperations were then divided into those occurring for acute postoperative complications such as infection, wound related issues, or malpositioned hardware versus those outside the acute postoperative period. 
Results: Demographics are presented in Table 1 and Ranawat class in Table 2. 222 of 266 patients $(83 \%)$ had a minimum two-year follow up with an average follow up of 4.97 years. Overall, 26 patients required 30 reoperations $(13.5 \%) .15$ patients required 16 reoperations $(7.2 \%)$ in the acute postoperative period: 10 patients $(4.5 \%)$ for infection requiring at least one irrigation and debridement, $3(1.3 \%)$ patients for hardware related issues, and $3(1.3 \%)$ patients for posterior cervical wound issues, one of which was a CSF fistula. 13 patients required 14 reoperations $(6.3 \%)$ outside of the acute postoperative period: $6(2.7 \%)$ for the development of a new radiculopathy, 3 $(1.3 \%)$ for recurrent myelopathy, $2(0.90 \%)$ for the development of neurological symptoms with a kyphotic deformity and $1(0.45 \%)$ for a post-traumatic focal kyphotic deformity. 2 patients each reported an additional procedure being performed at an outside hospitals but the records were unable to be reviewed. Patients who had a concomitant laminectomy, either partial or complete, demonstrated a significantly $(p=0.03)$ higher reoperation rate compared to those that did not. This remained significant when comparing only the late reoperation cohort $(\mathrm{p}<0.008)$. No other statistically significant associations were found. We had an $18 / 222(8.1 \%)$ C5 palsy rate.

Conclusion: Our cohort had a $13.5 \%$ reoperation rate with a $6 \%$ reoperation rate outside of the acute postoperative period related to the development of new neurological symptoms. Given the preservation of motion and less invasive nature of laminoplasty, these results support this procedure as a reasonable alternative to laminectomy and fusion.

\section{Presentation \#64. Impact of Body Mass Index on Surgical Outcomes, Narcotic Consumption, Costs and Reimbursements following Anterior Cervical Discectomy and Fusion}

Kern Singh, MD, Chicago, IL; Benjamin C. Mayo, BA, Chicago, IL; Dustin H. Massel, BS, Northbrook, IL; Krishna Modi, BS, Schaumburg, IL; William W. Long, BA, Willoughby, OH; Jonathan S. Markowitz, BS, Teaneck, NJ; Jacob V. DiBattista, BS, Chicago, IL

Introduction: Obesity has often been associated with worse outcomes following spine surgery. Patients with greater BMIs may require more postoperative narcotics, increasing their risk for side effects and toxicity. Few studies have examined the effect of BMI classification on postoperative outcomes, narcotic consumption, complications, incidence and prevalence of revision surgery, costs or reimbursements following a 1- or 2-level ACDF. The purpose of this study is to compare surgical outcomes, postoperative narcotic consumption, complications, costs, and reimbursements across body mass index (BMI) stratifications for patients undergoing primary 1- or 2-level anterior cervical discectomy and fusion (ACDF).

Materials and Methods: A prospectively maintained surgical database of patients that underwent a primary 1- or 2-level ACDF for degenerative spinal pathology between 2007 - 2013 was reviewed. Patients were stratified in to one of four groups according to BMI: normal weight $(<25 \mathrm{~kg} / \mathrm{m} 2)$, overweight $(25-30 \mathrm{~kg} / \mathrm{m} 2)$, obese I $(30-35 \mathrm{~kg} / \mathrm{m} 2)$, or obese II-III ( $\geq 35 \mathrm{~kg} / \mathrm{m} 2)$. Differences in patient demographics and preoperative characteristics were compared across the cohorts using independent sample t-tests and Chi-square analysis. The effect of BMI on peri- and postoperative outcomes analyzed using multivariate linear and logistic regression adjusted for demographic, comorbidity, and procedural characteristics. Two cohorts of 30 patients were matched for number of fusion levels, smoking, and Charlson Comorbidity Index (CCI) score to compare hospital costs and reimbursements. OME means were compared utilizing nonparametric analysis to adjust for distortions.

Results: A total of 315 patients were included in the analysis, of which $72(22.9 \%)$ were normal weight, $117(37.1 \%)$ were overweight, $72(22.9 \%)$ were obese I, and $54(17.1 \%)$ were obese II-III. No difference in age, gender, smoking status, operative level, or preoperative VAS was found between cohorts (Table 1). However, greater BMI was associated with having an increased comorbidity burden ( $\mathrm{p}<0.001)$. BMI was not found to be associated with mean operative time, estimated blood loss (EBL), length of hospital stay (LOS), in-hospital narcotic consumption, postoperative VAS scores at 6-weeks, 3-months, or 6-months, narcotic dependence at the 1st or 2nd postoperative visit, or complication and arthrodesis rates (Table 2 ). In the matched cohort analysis, the payments to charge ratios were $0.57 \pm 0.36$ and $0.70 \pm 1.7$ in the obese and non-obese cohorts, respectively, and no significant differences existed with regards to charges, reimbursement, or direct costs between cohorts $(\mathrm{p}>0.05)$.

Conclusions: Patients with increased BMIs demonstrated comparable surgical outcomes, narcotic consumption, and hospital costs when compared to those with lower BMIs. While obese patients may present a technical challenge to spine surgeons, obesity was not associated with increased postoperative pain, narcotic consumption, complication rates, or hospital expenses. As such, the decision to perform a primary 1- or 2- level ACDF may not differ across BMI stratifications.

Presentation \#65. Effect of Surgical Setting (Tertiary vs. Community Hospitals) on Hospital Reported Outcomes for Anterior Cervical Spine Procedures

Eugene Koh, MD, PhD, Baltimore, MD; Ehsan Jazini, MD, Baltimore, MD; Neil Sardesai, MD, Baltimore, MD; Tristan Weir, BS, Baltimore, MD; Kelley E. Banagan, MD, Baltimore, MD; Daniel Gelb, MD, Baltimore, MD; Steven C. Ludwig, MD, Baltimore, MD

Introduction: As hospital compensation becomes dependent on pay-for-performance and bundled payment compensation models, hospitals seek to reduce costs and improve patient outcomes by decreasing patient length of stay (LOS), potentially avoidable utilizations (PAUs, 30 day all-cause readmissions / revisits), and in-hospital provider preventable conditions (PPCs, as defined by CMS, including postoperative respiratory failure, renal failure, decubitus ulcer, postoperative wound infection, and reoperation, among others). We sought to evaluate hospital-reported outcomes measures for elective ACDF at a tertiary hospital (TH) versus community hospitals $(\mathrm{CH})$ within the same hospital system. The purpose was to determine if elective ACDFs performed at THs versus CHs have different LOS, PPCs, and PAUs.

Materials and Methods: 698 consecutive patients (January 2015 - January 2016) undergoing an ACDF were retrospectively reviewed from a physician-driven, prospective database of a single medical system consisting of one $\mathrm{TH}(\mathrm{N}=97)$ and four $\mathrm{CHs}(\mathrm{N}=601)$. Inclusion criteria consisted of patients ( $>18$ years old) who underwent elective ACDF. Exclusion criteria included: trauma, tumor, and infection. Independent variables included: age, sex, ethnicity, insurance type, sub-specialty (orthopaedic spine surgery or neurosurgery), number of fusion levels, use of instrumentation plate, inpatient status, and discharge disposition. The primary outcome was the mean LOS. Secondary outcomes included rates of PPCs and PAUs.

Results: Table 1 shows the patient characteristics between THs and $\mathrm{CHs}$. The $\mathrm{CH}$ patients were significantly older $(\mathrm{P}=0.003)$ and were predominantly white. $\mathrm{CHs}$ had fewer patients with medicare / medicaid / medical assistance and more self-payers than the TH. THs had a higher proportion of orthopaedic surgeons performing ACDFs. CHs performed a greater number of fusion levels compared to the TH (mean, $2.23 \pm 0.99$ vs. $1.79 \pm 0.93, \mathrm{P}<0.001$ ). Significantly more patients were admitted postoperatively in the CHs vs. the $\mathrm{TH}$ $(70.2 \%$ vs. $65.9 \%, \mathrm{P}<0.001)$. The $\mathrm{TH}$ discharged patients to home proportionally more than the $\mathrm{CHs}(92.8 \%$ vs. $70.2 \%, \mathrm{P}<0.001)$. Table 2 shows the univariate and multivariate linear regression results for predictors of LOS. After adjusting for age, sex, ethnicity, insurance type, specialty, surgical factors, inpatient status, discharge disposition, and PPCs, the TH was associated with a 0.51 days greater LOS $(\mathrm{P}$ $=0.017 ; 95 \%$ CI, $0.09-0.94)$ compared with the CHs. The most significant predictors of increased LOS were the presence PPCs and 
discharge to a facility (4.41 and 1.80 days longer LOS, respectively; $\mathrm{P}$ $<0.001)$. Medicare / Medicaid insurance significantly increased LOS by 0.37 days.

Conclusion: In the multivariate analysis we found that tertiary vs. community setting, age, medicaid / medicare status, sub-specialty (neurosurgery vs. orthopaedics), and the presence of a PPC had significant correlation with a longer LOS. Surprisingly, the number of levels fused did not predict a longer LOS. The presence of a PPC had the most acute effect on prolonging LOS which highlights the importance in finding strategies to help mitigate it. This surgeon-driven data may help develop more effective protocols to decrease LOS while minimizing PAUs and PPCs. This could potentially improve patient care, reduce hospital costs, and improve surgeon compensation.

\section{Presentation \#66. Predictive Models for Patient-Centered Efficacy} and Discharge Destination after Elective Cervical Spine Surgery

Ahilan Sivaganesan, MD, Nashville, TN; Silky Chotai, MD, Nashville, TN; Elliott J. Kim, MD, Nashville, TN; David Stonko, BS, MS, Nashville, TN; Joseph Bradley Wick, BA, Nashville, TN; Matthew McGirt, MD, Charlotte, NC; Clinton J. Devin, MD, Nashville, TN

Introduction: Surgery is a valuable therapeutic option for degenerative cervical spine disease, however there is uncertainty as to which patients benefit. Here we introduce predictive models for clinically meaningful improvement in disability, as well as discharge destination, after cervical spine surgery (CSS).

Methods: 430 patients undergoing CSS were enrolled into a prospective registry. LOESS regression was performed to verify that a linear relationship between 12-month Neck Disability Index (NDI) and various explanatory variables was reasonable. The following variables were used to power a multiple linear regression model for NDI: demographics, diagnosis, number / location of diseased levels, baseline symptoms and PROs, employment / insurance status, comorbidities, a history of prior surgeries, and surgical approach. Possible interactions among variables such as diagnosis, age, baseline NDI, and employment status were also accounted for in the analysis. We then used Repeated Random Sub- Sampling (related to Monte Carlo cross-validation) to validate the predictive performance of our model. A separate model, based on logistic regression, was constructed to predict a clinically important improvement in NDI (at least 17.3) at one year. A third model was also developed and validated, using similar methods, with the aim of predicting post-surgery discharge destination (home versus facility).

Results: The mean NDI one year after surgery was 25.82, and the mean improvement was 16.33 points. $48 \%$ (205) of patients achieved the minimum clinically important difference (MCID) in NDI. Our predictive model for 12-month NDI has an R-squared of 0.69 (observed versus predicted NDI scores are plotted in Figure 1), and in validation, it achieved an R-squared of 0.43 . The predictors, in descending order of influence, are: employment, baseline NDI, diagnosis, smoking, ethnicity, claudication, narcotic use, and symptom duration. Our model for achieving a MCID in NDI has an area under the curve greater than 0.80 for the development phase and an AUC of 0.65 for the validation phase. The predictors, in descending order of influence, are: baseline NDI, motor deficit, depression, ambulation, revision surgery, employment, diagnosis, smoking, and symptom duration. Finally, our predictive model for discharge destination has an area under the curve greater than 0.80 for the development phase and an AUC of 0.75 for the validation phase (ROC curve shown in Figure 2). The predictors, in descending order of influence, are: baseline EQ-5D, number of levels, myelopathy, depression, baseline NDI, and motor deficit.

Conclusion: We present internally validated models that can help predict disability at one year, clinically meaningful improvement in disability, and discharge destination after elective CSS. Our NDI model explains roughly $70 \%$ of the variation in 12-month neckrelated disability. The predictive accuracy of our associated model for achieving a MCID in NDI is a good starting point, but leaves room for improvement. Our model for discharge destination has strong predictive accuracy, and with external validation at other institutions, it can become a useful tool as spine care providers seek to better understand the post- operative trajectories of their patients.

Presentation \#67. Reimbursement and Charges Related to a 90-Day Episode of Care for a One- or Two-Level Anterior Cervical Discectomy and Fusion

Sohrab S. Virk, MD, MBA, Columbus, OH; Frank M. Phillips, MD, Chicago, IL; Safdar N. Khan, MD, Columbus, $\mathrm{OH}$

Introduction: Bundled payments represent a single payment for services during an episode of care for a surgical procedure. Anterior cervical discectomy and fusion (ACDF) and associated 90-day costs have been suggested as a "bundle" amenable to such a payment structure; however, little data regarding costs related to this procedure and subsequent care are available.

Methods: The Medicare 5\% national sample administrative database was used to catalog clinical and financial data associated with the day of surgery and the 90-day postoperative period for patients undergoing a one- to two-level ACDF procedure from 2005 to 2012. We simultaneously queried the database for total knee replacement as a means to compare the payments and verify the reliability of our analysis.

Results: A total of 4,506 patients underwent an ACDF procedure for cervical radiculopathy. Total 90 -day reimbursement was $\$ 69,469,550$ or $\$ 15,417$ / patient $(+/-\$ 947$, median $=\$ 15,589)$. As a comparison, reimbursement for TKR patients amounted to $\$ 17,451 /$ patient. Physician reimbursement for ACDF represented $20.42 \%$ of the total with the surgeon receiving $18.07 \%$ of total reimbursement. Revision surgery, readmission and emergency department reimbursement accounted for $0.71 \%$ of total reimbursement. Reimbursement for rehabilitation service, including physical therapy, skilled nursing facilities and home care represented $3.11 \%$ of total reimbursement. There was a statistically significant variation in reimbursement among geographic regions, being highest in the western United States $(\mathrm{p}=0.015)$ [Figure 1], and a trend towards increase in overall reimbursement over the years from 2005 to $2012(\mathrm{p}=0.082)$ [Figure 2].

Conclusions: This study is the first report we are aware of 90-day reimbursement / patient for one- to two-level ACDF procedures in a Medicare cohort. Payments had a statistically significant variation among geographic locations. Our study provides a reimbursement benchmark for one- to two-level ACDF procedures and understanding the payments relative to costs will help providers understand whether a bundled payment for the ACDF procedure is economically viable.

\section{1st Place Clinical Paper Award • \$2,000}

Presentation \#68. Is there Value in Retrospective 90-Day Bundle Payment Models for Cervical Spine Procedures?

Susan M. Odum, PhD, Charlotte, NC; Bryce Allen Van Doren, MA, MPH, Charlotte, NC; Leo R. Spector, MD, Charlotte, NC

Introduction: The Centers for Medicaid and Medicare Services (CMS) Bundled Payments for Care Improvement (BPCI) initiative was implemented in 2015. At our private practice, we implemented a retrospective payment model 2 for a 90-day episode of care for cervical 
spine and other orthopedic procedures. Under these retrospective payment models, Medicare continues to make fee-for-service (FFS) payments but reconciles the total expenditures for the episode with a bundled payment amount as determined by CMS. A payment or recoupment amount is then made by Medicare reflecting the aggregate expenditures compared to the target price. The purpose of the study is to assess the value of the cervical spine CMS bundle at our private practice.

Methods: We utilized the data provided by CMS to compare the total expenditures of cervical spine diagnosis related groups (DRGs) of 471, 472, and 473. Medicare patients who underwent cervical spine surgery between January 2009-December 2012 were defined as nonBPCI $(\mathrm{n}=88)$ and were compared to Medicare BPCI patients $(\mathrm{n}=40)$ who had surgery between January 2015-December 2015. Post-acute events within the 90 day episode including admission to an IRF or SNF as well as home health $(\mathrm{HH})$ and readmissions were analyzed. Expenditures were converted to 2016 dollars using Consumer Price Index (CPI). Normality of expenditures was assessed using the Manning \& Mullahy method and expenditures were subsequently log transformed. Wilcoxon tests and a multivariate generalized estimated equation were used to determine differences between BPCI and nonBPCI patients as well as assess the independent effects of post-acute events.

Results: The median expenditure for non-BPCI patients was \$16,566 (IQR \$14,604 - \$19,951) compared to \$18,510 (IQR \$15,936$\$ 23,371)$ for BPCI patients $(\mathrm{p}=.02)$. Compared to non-BPCI patients BPCI patients had a higher rate of SNF admissions (non-BPCI $6 \%$ vs $7.5 \%$ BPCI; $p=.23$ ), IRF admissions (non-BPCI $1 \%$ vs. $5 \%$ BPCI; $p$ $=.68), \mathrm{HH}$ (non-BPCI 14\% vs. 15\% BPCI; $\mathrm{p}=.79$ ) and readmissions (non- BPCI $9 \%$ vs. $12.5 \%$ BPCI; $p=.54$ ). At the multivariate level, the significantly higher expenditure for BPIC patients persisted and all post acute events were significant, independent drivers of increased cost. After controlling for post acute events, BPCI patients had a $10 \%$ increase in expenditures $(\mathrm{p}=.02)$. Admissions to an IRF or SNF increased cost 93\% $(\mathrm{p}<.001)$ and $56 \%(\mathrm{p}<.001)$, respectively. $\mathrm{HH}$ utilization increased expenditures $26 \%(\mathrm{p}<.0001)$ and 90 -day readmissions increased costs by $45 \%(\mathrm{p}<.0001)$.

Conclusion: The objective of the BPCI initiative was to improve the value of health care, e.g. decreasing cost while improving outcomes. Our institution was only managing the post acute care expenditures and not the acute hospital expenditures. In spite of our best efforts to contain costs with clinical practice guidelines, patient navigators and a BPCI management team, the expenditures were significantly higher for BPCI patients. Furthermore, the outcomes defined as post acute events were not improved. The variability of surgical procedure complexity included in the 471, 472 and 473 DRGs, cervical spine bundles may not be appropriate. We have discontinued BPCI for cervical spine DRGs and are focusing our efforts on defining bundles by specific Current Procedural Terminology ${ }^{\circledR}$ codes.

\section{Presentation \#69. The Use of a Novel iPad Application to Quantify Dysfunction in Cervical Myelopathy Patients}

Tyler J. Jenkins, MD, Chicago, IL; Brett D. Rosenthal, MD, Chicago, IL; Arjun Ranade, BS, Brookfield, WI; Surabhi A. Bhatt, BS, Chicago, IL; Wellington K. Hsu, MD, Chicago, IL; Alpesh A. Patel, MD, River Forest, IL

Introduction: Cervical myelopathy is the leading cause of spinal cord dysfunction in the adult population. Despite the prevalence and importance of the condition there is a paucity of objective and quantitative clinical measures for analysis of the disease process. The most common diagnostic tools available to the physician are non-quantitative physical exam findings (pathologic reflexes, gait disturbance) and subjective scoring systems (mJOA, Nurick scales). The lack of an easily-performed, objective, and quantitative diagnostic tool has hindered the diagnosis of these patients. In an effort to better classify myelopathy, provide earlier diagnosis, and improve clinical outcome measurements we developed a novel iPad application to test fine motor skills. A decline in these fine motor skills is an early hallmark of cervical myelopathy. We wrote a novel code for the iPad application with clinical application in mind and thus were able to fine tune the instrument making use efficient for clinicians and patients.

Methods: We recruited 71 healthy control patients and 8 myelopathic patients aged of at least 18 years and no neurologic or physical condition (i.e. Parkinson's, dementia, blindness, rheumatoid arthritis) that precluded fine motor testing. Myelopathic subjects were diagnosed by a fellowship trained spine surgeon based on clinical and radiographic evaluation. Enrolled patients completed the modified Japanese Orthopaedic Association scale (mJOA) for cervical myelopathy and our novel Fine Motor Skills (FiMS) iPad application. The FiMS iPad application consists of 4 unique challenges (Figure 1). All the challenges focus on the use of fine motor dexterity testing and increase in difficulty as the challenges progress. Challenge 1 involves accurately tapping a moving target on the screen. Challenge 2 necessitates dragging a target on the screen to a goal. Challenge 3 involves moving a target through a maze without touching the maze walls. Challenge 4 is similar to Challenge 2 but requires the use of both hands to drag 2 separate targets to a goal. The scores are recorded independently for each challenge and the mean scores were used for data analysis. A student t-test was used to determine significance with a p-value set at $<0.01$.

Results: The average mJOA score (scale $0-18$ ) for the myelopathic cohort was 11.4 with a score less than 12 being classified as severe myelopathy. The 71 control patients had a mean mJOA score of 17.4 with a score greater than 17 being inconsistent with myelopathy. Regression analysis of the healthy controls $(n=71)$ showed that FiMS challenge scores decreased with age in all four challenges. When compared to age-matched healthy controls $(n=44)$ the myelopathic cohort had significantly lower FiMS scores for all challenges 1-4 (Table 1).

Conclusion: The novel Fine Motor Skills (FiMS) iPad application produced significantly lower scores in a myelopathic cohort when compared to an age-matched control cohort. This is true for all 4 challenges in the FiMA iPad application. In summary, the FiMS iPad application is a novel, easily administered, objectively quantifiable test for analyzing cervical myelopathy.

Presentation \#70. Association between Paraspinal Muscle
Morphology, Clinical Symptoms and Functional Status in Patients
with Degenerative Cervical Myelopathy

Octavian Dobrescu, Cote St. Luc, QC, Canada; Matthew Courtemanche, Surrey, BC, Canada; Carolyn J. Sparrey, PhD, Surrey, BC, Canada; Michael G. Fehlings, MD, PhD, Toronto, ON, Canada; Michael H. Weber, MD, Montreal, QC, Canada; Carlo Santaguida, FRCSC, MD, Montreal, QC, Canada

Introduction: Cervical muscle alterations have been reported in patients with chronic neck pain, but the assessment of cervical muscle morphology has been overlooked in patients with degenerative cervical myelopathy (DCM). The objective of this study was to assess the composition (e.g. fatty infiltration) and asymmetry of the multifidus (MF), semispinalis cervicis (SCer), semispinalis capitis (SCap) and splenius capitis (SPL) muscles in patients with DCM and evaluate their correlations with clinical signs, symptoms and functional scores.

Materials and Methods: Thirty-eight patients diagnosed with DCM and spinal cord compression at C4-C5 $(n=20)$ or C5-C6 $(n=$ 18) (first level of compression) were selected from the AOSpine CSM database. Cervical muscle measurements of cross-sectional area (CSA) (Figure 1) and ratio of functional CSA (fat free area, FCSA) to total CSA (Figure 2) were obtained from T2-weighted axial MR images at the level above, same, and level below the most cranial level of spinal cord compression. Muscle fatty infiltration and asymmetry was 
assessed at every level and their associations with respect to clinical signs, symptoms and functional scores were investigated.

Results: There was a significant increase in fatty infiltration of the MF $(p=0.001)$ and SPL $(p<0.001)$ muscles at the level below the compression. A significant increase in MF CSA asymmetry was also observed at the level below the compression. Lower MF FCSA / CSA ratio was associated longer 30-meter walking test time. Lower SCer FCSA / CSA was associated with corticospinal distribution motor deficits and atrophy of the hands. Greater asymmetry in SCap CSA was associated with higher Neck Disability Index (NDI) scores while lower asymmetry in MF CSA was associated with a positive Hoffman sign and weakness.

Conclusion: A significant increase in muscle fatty infiltration and CSA asymmetry at the level below the compression was observed in patients with DCM. Our results also suggest an association between cervical muscle morphology and DCM clinical signs, symptoms and functional status. Clinicians should pay greater attention to cervical muscle morphology and composition in patients diagnosed with DCM and further evaluate whether such muscle parameters have an impact on prognosis and functional recovery.

\section{Presentation \#71. MRI Analysis of the Combined AOSpine North America and International Studies: The Prevalence and Spectrum of Pathologies in a Global Cohort of Patients with Degenerative Cervical Myelopathy}

Aria Nouri, MD, MSc, Richmond, ON, Canada Allan Martin, MD, Toronto, ON, Canada; Lindsay A. Tetreault, PhD, Oakville, ON, Canada So Kato, MD, Tokyo, Japan; Hiroaki Nakashima, MD, PhD, Nagoya, Japan; Narihito Nagoshi, MD, PhD, Shinjuku-ku Tokyo; Hamed Reihani-Kermani, MD, Toronto, ON, Canada; Michael G. Fehlings, MD, PhD, Toronto, ON, Canada

Introduction: Degenerative Cervical Myelopathy (DCM) encompasses a spectrum of age-related conditions of the cervical spine, including spondylosis, which result in progressive spinal cord injury through static and dynamic injury mechanisms. Unfortunately, little is known of the prevalence and constellation of anatomical pathology that presents in these patients and if there are differences between genders. Through detailed review of MRIs from prospective AOSpine multicenter studies, it is the purpose of the present research to report on the global prevalence of degenerative cervical pathologies of surgically treated DCM patients. Such information would be potentially helpful in uncovering etiological factors, provide insight into the natural history, and determine risk factors for DCM.

Methods: MRIs of 458 patients were reviewed for the type of pathology, source of stenosis, level of maximum cord compression, levels of spinal cord compression (SCC), and signal changes on T2WI and T1WI. Additionally, a cord occupying ratio (COR) within the canal at non-compressed sites was calculated and a COR $\geq 70 \%$ was used to identify congenital stenosis. The prevalence of these changes was separated into genders and the proportions were assessed using Chi-square analysis. A p-value of $\leq 0.05$ was considered as statistically significant. Additionally, the proportion of degenerative changes present alongside other diagnoses was computed as well as the prevalence of pathologies per geographical region.

Results: Globally, spondylosis was the most frequent cause of SCC $(89.7 \%)$ and was frequently accompanied by enlargement of the ligamentum flavum (LF) (59.8\%), Figure 1. OPLL was accompanied by spondylosis in $91.7 \%$. Single level disc pathology, OPLL and spondylolisthesis had a prevalence of $\sim 10 \%$. Associated abnormalities such as Klippel-Feil Syndrome and congenital stenosis were observed in $2.8 \%$ and $8.4 \%$, respectively. Single level disc pathology was less common in North America, congenital stenosis less common in Europe, and OPLL more common and spondylolisthesis less common in Asia- Pacific. Females presented more commonly with single level disc pathology $(p=0.013)$ and were less likely to have their maximum site of cord compression at C3-4 $(\mathrm{p}=0.007)$. Males more commonly presented with spondylosis $(p=0.017)$ and enlargement of LF $(p=$ 0.012). Globally, the C5-6 region was the most frequent maximum compressed site (39.7\%) and region for T2WI hyperintensity (38.9\%). T2WI hyperintensity more commonly presented in males $(\mathrm{p}<0.001)$.

Conclusion: DCM presents as a constellation of pathologies that most commonly includes multilevel disc and bone pathologies, as well as enlargement of the LF. These findings support that pathological features, including OPLL, are highly interrelated with one another and rarely present in isolation. There appears to be a number of differences in the frequency and constellation of pathologies between genders. Overall, females presented with milder degenerative changes and correspondingly a lower frequency of T2WI hyperintensity of the spinal cord on MRI. There are also variances in the spectrum and prevalence of pathologies between geographical regions and these may be due to a multitude of causes that likely span beyond ethnic factors. Presentation \#72. High-Resolution Magnetization Transfer (MT)
MRI in Patients with Cervical Spondylotic Myelopathy

Brett D. Rosenthal, MD, Chicago, IL; Linda I. Suleiman, MD, Chicago, IL; Kenneth A. Weber II, DC, Chicago, IL; Jason Savage, $M D$, Chicago, IL; Wellington Hsu, MD, Chicago, IL; Todd Parrish, PhD, Chicago, IL; Alpesh A. Patel, MD, River Forest, IL

Introduction: Cervical spondylotic myelopathy (CSM) is a progressive degenerative disease of the spine that has significant clinical morbidity with significant variation in symptoms. Advanced imaging with magnetic resonance imaging (MRI) has largely been accepted as a tool to evaluate CSM. However, there are limitations on quality and predictability of clinical deterioration, as such MRI now only serves to confirm the diagnosis. Magnetization transfer (MT) is a technique based on the application of off- resonance radiofrequency pulses and observing their effects on MR images. MT contrast has been established as a marker of myelin integrity through its ability to measure the exchange of freely moving protons to large macromolecules. We sought to compare the magnetization ratio (MTR) in healthy subjects to CSM patients.

Materials and Methods: Seven healthy controls and ten patients with clinical and MRI imaging manifestations of CSM were identified by three board-certified spine surgeons. The severity of CSM was assessed with the Nurick score. For imaging, transverse slices across the intervertebral discs of the cervical spine were acquired using a gradient echo sequence with and without an MT saturation pulse on a 3 Tesla Siemens Prisma scanner $(\mathrm{TR}=300 \mathrm{~ms}, \mathrm{TE}=17 \mathrm{~ms}$, flip angle $=30^{\circ}$, in-plane resolution $=0.47 \times 0.47 \mathrm{~mm} 2$ ). Image processing was performed using the Spinal Cord Toolbox. The MT1 and MT0 images were coregistered, and MTR images were calculated. A T2 anatomical image of the cervical spine, which was in alignment with MT images, was normalized to a standard spinal cord template (Figure 1A), and the output warping fields were used to transform the MT images to standard space and transform a spinal cord mask from standard to native space. Using the transformed mask, the mean MTR was calculated at each intervertebral disc level (Figure 1B).

Results: The mean MTR across all of the intervertebral disc levels was $34.8 \pm 3.5$ (mean \pm standard deviation) for the controls and $30.4 \pm 6.5$ for the CSM patients. The CSM patients tended to have a lower mean MTR than the controls, but the difference was not significant (independent samples t-test, $\mathrm{p}=0.110$ )(Figure $2 \mathrm{~A}$ ). The mean MTR across all of the intervertebral disc levels was not significantly correlated to the Nurick score (Spearman's $\rho=-0.489, \mathrm{p}=$ 0.151 )(Figure 2B). However, when focusing only at the intervertebral disc level with the lowest MTR for each subject, the mean MTR at this level was negatively correlated to the Nurick score (Spearman's $\rho=-0.725, p=0.018$ ) (Figure 2C).

Conclusion: CSM patients tended to have decreased MTR indicating myelin degradation compared to our healthy subjects, and 
MTR was negatively correlated with the severity of CSM. MT MRI may have the potential to better detect structural changes in white matter than conventional $\mathrm{T} 1$ and $\mathrm{T} 2$ imaging techniques.

Presentation \#73. The K-line Tilt, a Novel Radiographic Parameter
of Cervical Sagittal Balance, is a Predictor of Postoperative
Kyphotic Deformity after Laminoplasty for Cervical Myelopathy Caused by Ossification of the Posterior Longitudinal Ligament

Kenichiro Sakai, MD, PhD, Saitama, Japan; Toshitaka Yoshii, MD, PhD, Tokyo, Japan; Takashi Hirai, MD, PhD, Tokyo, Japan; Yoshiyasu Arai, Saitama, Japan; Yu Matsukura, MD, PhD, Saitama, Japan; Astushi Okawa, MD, PhD, Tokyo, Japan

Introduction: Cervical laminoplasty (LAMP) is a standard surgical procedure for patients with cervical myelopathy caused by ossification of the posterior longitudinal ligament (C-OPLL). However, it is well known that LAMP is not suitable for patients with massive OPLL lesions or cervical kyphotic alignments. Moreover, one of the important complications following LAMP is postoperative kyphotic deformity, which prevents posterior spinal cord shift and leads to postoperative residual anterior compression of the spinal cord. While the K-line, which can evaluate OPLL size and cervical alignment in one parameter, is a good clinical tool for making decisions about surgical procedures, it cannot predict the postoperative kyphotic deformity following LAMP. Recently, it was reported that preoperative cervical sagittal imbalance is a predictive factor for postoperative kyphotic deformity following LAMP. We proposed the 'K-line tilt', a novel radiographic parameter of cervical sagittal balance, and hypothesized that it may influence the occurrence of postoperative kyphotic deformity following LAMP.

Materials and Methods: The study included a total of 38 consecutive patients ( 27 male, 11 female; mean age 65.4 years) who underwent LAMP for C-OPLL at our hospital from 2008 and completed at least 1 year of follow-up. We performed LAMP only for patients with slight OPLL lesions and without cervical kyphotic alignment. The average follow-up period was 3.1 years. We defined the K-line tilt as an angle between the $\mathrm{K}$-line, which connects the midpoints of the spinal canal at $\mathrm{C} 2$ and $\mathrm{C} 7$, and the vertical line (Figure 1). Cervical lateral X-ray images taken in the neutral standing position were evaluated preoperatively and at the final follow-up visit. Radiographic measurements included the following: (1) K-line (2) K-line tilt, (3) CGH-C7 SVA, (4) CL (C2-7 lordotic angle) and (5) C7 slope. Clinical results were evaluated using the Japanese Orthopedic Association scoring system for cervical myelopathy (C-JOA score).

Results: The K-line tilt was strongly correlated with the CGH-C7 SVA preoperatively $(\mathrm{R}=0.842)$ and postoperatively $(\mathrm{R}=0.845)$. Preoperatively, all 38 patients had non-kyphotic cervical alignment and $\mathrm{K}$-line $(+)$; however, kyphotic deformity $\left(\mathrm{CL}<-5^{\circ}\right)$ was observed in 5 patients and $\mathrm{K}$-line (-) in 6 patients at the final follow-up. We compared preoperative factors between the kyphotic deformity group (5 cases) and the non-kyphotic deformity group (33 cases). Preoperative K-line tilt was significantly different $(\mathrm{P}<0.01)$, but age, the $\mathrm{CL}$ and the $\mathrm{C} 7$ slope were similar between the two groups. The recovery rates of the C-JOA scores at the final follow-up in the kyphotic deformity group were worse than those in the non- kyphotic deformity group (14.1\% vs. 46.6\%: $\mathrm{P}<0.05$ ). $\mathrm{K}$-line tilt was determined to be a preoperative risk factor using multivariate analysis $(\mathrm{P}=0.014, \mathrm{OR}=1.366)$. The cutoff value by ROC analysis was a K-line tilt of $20^{\circ}$, which was associated with $80.0 \%$ sensitivity and $93.9 \%$ specificity, for predicting kyphotic deformity.

Conclusion: K-line tilt is a predictive factor for postoperative kyphotic deformity after LAMP for C-OPLL patients, and LAMP is not suitable for patients with a K-line tilt $\geq 20^{\circ}$, even in cases with normal preoperative alignment and slight OPLL lesions.
Presentation \#74. The Difference in Clinical Outcomes between ACDF and Posterior Foraminotomy in Professional Athletes

Harry T. Mai, MD, Manhattan, CA; Andrew D. Schneider, BA, Chicago, IL; Sean M. Mitchell, MD, Chicago, IL; Jason W. Savage, MD, Chicago, IL; Alpesh A. Patel, MD, River Forest, IL; Andrew C. Hecht, MD, New York, NY; Wellington K. Hsu, MD, Chicago, IL

Introduction: Excellent clinical outcomes after operative management of cervical disc herniation $(\mathrm{CDH})$ have been reported, however, there is has been no comparative study to guide a surgeon's choice of particular operative procedure in the professional athlete. Anterior cervical discectomy and fusion (ACDF), posterior foraminotomy $(\mathrm{PF})$ have all been reported to have excellent clinical outcomes in the general population but the intense physical regimen of the professional athlete necessitates different outcome measures specific to his sport.

Materials and Methods: Professional athletes of the four major professional sports leagues - National Football League (NFL), Major League Baseball (MLB), National Hockey League (NHL) and National Basketball Association (NBA) - diagnosed with CDH and managed operatively were identified through team injury reports and archives on public record through a previously established protocol. Athletes were grouped into cohorts based on the type of operation (ACDF and PF). Outcome measures including games played, games started, seasons played and sport specific statistics were compared in each cohort before and after surgery. As used in previously established protocols, athlete performance score based on sport specific statistics was calculated and standardized for comparison across the sports.

Results: A total of 101 professional athletes met the inclusion criteria; 86 underwent ACDF, 13 underwent PF and 2 underwent TDA. The PF cohort had a significantly greater rate of return to play $(92.3 \%$ vs. $70.9 \%, p=.03)$ and the shortest time to return after surgery ( 238 vs. 367 days, $p=.0345$ ) [Table 1]. However, the reoperation rate at the index level was significantly higher for PF patient compared to $\operatorname{ACDF}(46.2 \%$ versus $1.2 \%$, respectively) $(p=.0001)$. While there was an overall decrease in performance score after surgery, there was not significant difference between the surgical cohorts $(\mathrm{p}=.336)$. There was also no difference in long-term survival $(\mathrm{p}=0.11)$ [Figure 1].

Conclusion: Anterior cervical discectomy and fusion, posterior foraminotomy both represent viable options for the operative management of $\mathrm{CDH}$ in the professional athlete. The role of total disc arthroplasty in elite athletes remains to be determined and may depend on the particular sport. Posterior foraminotomy provides athletes with significantly higher rate of return to play and quicker time to return compared to ACDF. However, athletes who undergo posterior foraminotomy must accept the significantly higher risk for reoperation at the index level.

Presentation \#75. The Accuracy and Safety of Subaxial Cervical Pedicle Screw Insertion using Vertebral Lateral Notch-Referred Technique

Kai Cao, MD, PhD, Nanchang, China; Chunyang Wu, MD, Nanchang, China; Qingxiu Leng, MD, Nanchang, China; Zhimin Pan, MD, MSc, Nanchang, China; Jiaquan Luo, Nanchang, China; Pingguo Duan, Nanchang, China

Instruction: Biomechanical studies revealed that pedicle screw instrumentation has superior stabilizing effect than other internal fixations in the reconstruction of subaxial cervical spine, particularly, in the surgery of cervical tumor resection, deformity correction and severe fracture and dislocation. However, high neurovascular risk precludes surgeons to conduct the pedicle screw manipulation in cervical spine. We here advocate a novel, easy-mastering and practical technique (as called notch-referred technique) for subaxial cervical 
PS insertion. In this study, the accuracy and safety of vertebral lateral notch-referred technique for subaxial cervical pedicle screw (PS) were evaluated clinically.

Materials and Methods: Eighty-six consecutive patients with cervical disorders underwent cervical PS instrumentation in two spine teams in a single spine center. Preoperative $\mathrm{x}$-ray, CT and MRI of cervical spine were taken for surgery plan. The pedicle screw position was confirmed by postoperative CT scans. The penetration rate was analyzed and classified from ideal to unacceptable to assess the accuracy of this technique: grade $0=$ screw centered in pedicle; grade I = perforation of pedicle wall less than one-fourth of the screw diameter; grade II = perforation more than one-fourth of the screw diameter but less than one-second; grade III = perforation more than one-second outside of the screw diameter. Neurovascular complication related with PS insertion was recorded to assess the safety of this technique. The accuracy of PS placement between two surgeons was analyzed to confirm the manipulative consistency.

Results: A total of 504 pedicle screws were inserted in subaxial cervical spine. Postoperative CT scan indicated the accuracy of PS insertion by using notch-referred technique was $90.9 \%$ (458 / 504) (grade $0+$ grade I). There were no vertebral artery injury or spinal cord injury related with cervical PS misplacement in this cohort except one slight nerve root compression. The patient relieved from the radiculopathy in the course of follow-up without screw removal. No revisional surgery was conducted due to the misplacements of pedicle screws. The inter-surgeon consistency of inserting cervical PS was excellent $($ Kappa value $=0.86)$.

Conclusion: The vertebral lateral notch is the reliable and consistent anatomic landmark. The accuracy and safety of subaxial cervical pedicle screw insertion by using notch-referred technique are high and satisfactory. Notch-referred subaxial cervical PS insertion is an easymastering, practical technique.

Presentation \#76. Degenerative Cervical Spondylolisthesis: Does Adjacent Level Surgical Stabilization Result in Progressive Listhesis?

Grant D. Shifflett, MD, New York, NY; Jake T. Emerson, BS, Chicago, IL; Hollis E. Johanson, BS, Glenview, IL; Bryce A. Basques, MD, Chicago, IL; Jacob A. Birlingmair, BS, Chicago, IL; Dennis P. McKinney, BS, Chicago, IL; Po-Hsin Chou, MD, Taipei, Taiwan; Phil K. Louie, MD, Chicago, IL; Howard S. An, MD, Chicago, IL

Introduction: Degenerative cervical spondylolisthesis (DCS) occurs at a rate of $5.2 \%-11 \%$ in asymptomatic patients, increasing to up to $20 \%$ in symptomatic patients. Patients with cervical spondylotic myelopathy (CSM) often present with multi-level disease and may have a spondylolisthetic level within or adjacent to the levels of pathology. It remains unclear what happens to an unfused DCS segment when it is not included in a surgical construct. The primary aim of this investigation was to test the hypothesis that unfused DCS segments do not develop worsening instability requiring surgical intervention.

Materials and Methods: Twenty-four consecutive patients who presented with CSM, had radiographs revealing DCS at one or more levels, and underwent surgical intervention were retrospectively reviewed. All patients did not have clinical symptoms or radiographic pathology present at their DCS level and had surgery performed at adjacent levels. Demographic variables including age, sex, smoking, body mass index, number of levels fused, and location of levels fused, were documented. Radiographic measurements were obtained on pre-operative radiographs and final follow-up radiographs to assess the degree of instability, cervical lordosis, sagittal vertebral axis, and T1 slope at the affected level. Clinical and radiographic evaluation at final follow up was reviewed for signs of progression of disease and reoperation rates. Subgroup analysis was performed to assess for variables that might predict progression of unfused segments and reoperation rates.
Results: The cohort consisted of 13 males and 11 females. The average age at presentation was 61.3 years (range $27-83.6$ years). Mean follow-up was 21.4 months. DCS was present at C2-3 in three cases $(12.5 \%), \mathrm{C} 3-4$ nine cases $(37.5 \%), \mathrm{C} 4-5$ seven cases $(29.2 \%)$, C5-6 two cases $(8.3 \%)$, C6-7 no cases $(0 \%)$, C7-T1 three cases $(12.5 \%)$. Surgical procedures performed were as follows: anterior cervical discectomy and fusion $(18 ; 75.0 \%)$, posterior cervical fusion $(5 ; 20.8 \%)$, posterior cervical laminoplasty $(1 ; 4.2 \%)$. The average pre-operative slip was $2.7 \mathrm{~mm} \pm 0.6 \mathrm{~mm}$. At final follow-up, six $(25 \%)$ demonstrated progression $(>0.5 \mathrm{~mm})$ of their slip, $18(75 \%)$ remained stable $( \pm 0.5 \mathrm{~mm})$ or improved. The average slip at final follow-up was $2.9 \mathrm{~mm} \pm 1 \mathrm{~mm}$, which was not statistically significant $(\mathrm{p}=0.561)$. Three patients (12.5\%) with DCS developed symptomatic progressive instability with myelopathy or radiculopathy requiring further surgery. Multivariate analysis (Table 1) revealed age greater than 65 and female sex were associated with progression of listhesis. Radiographic parameters including level of listhesis, number of adjacent levels fused, cSVA, cervical lordosis, and T1 slope were not associated with progression. No demographic or radiographic variables were associated with reoperation.

Conclusions: The pathomechanics of DCS remain poorly understood and the necessity of including a spondylolisthetic level in a surgical construct remains in question. Despite the presence of increased stress at the DCS level due to adjacent surgical intervention, the majority of patients had the same or smaller slips at final follow-up and the majority of patients did not require further surgical intervention. Older age and female sex were associated with progression but did not increase the risk of reoperation. This study offers valuable information regarding the durability of a listhetic level adjacent to a surgical construct; however, further investigation with long-term follow-up is warranted.

Presentation \#77. Should Long Segment Cervical Fusions Be Routinely Carried into the Thoracic Spine? Multi-Center Analysis

Eeric Truumees, MD, Austin, TX; Devender Singh, PhD, Austin, TX; Matthew Geck, MD, Austin, TX; John Stokes, MD, Austin, TX

Introduction: While recommendations for caudal "end level" in posterior cervical reconstruction remain highly variable, the benefits of routine extension of posterior cervical fusions into the thoracic spine remain unclear. We compared clinical and radiographic outcomes in patients in whom posterior fusions ended in the cervical spine versus those in whom the fusion was extended into the thoracic spine. Our hypothesis was that extension of posterior cervical fusions into the upper thoracic spine improves clinical outcomes while decreasing kyphosis.

Methods: We assembled a multicenter (4 sites) radiographic and clinical database of patients that had undergone 3 or more level posterior cervical fusions for degenerative disease from January 2008 to May 2013 with at least 2 years of post-operative (post-op) follow-ups. Patients were divided into two groups: group I (fusion ending in the cervical spine) and group II (fusion extending into the thoracic spine). All radiographic measurements were performed by an independent experienced clinical researcher. Two-sample t-test with unequal variances was used to assess for differences between the two groups $(\alpha=0.05)$.

Results: Group I and Group II had 104 and 73 patients, respectively. The demographics of the two groups were similar. Minimum and maximum number of spinal levels treated for group I and group II were $3 \& 4$ and $3 \& 9$, respectively. Mean estimated blood loss (EBL) for group II was significantly higher than group I $(\mathrm{p}<0.05)$. Mean operative time (OR) and length of hospital stay (LOS) were comparatively higher for group II than group I but were not statistically significant $(p>0.05)$. Mean cervical lordosis at 2 years post-op improved in both groups. There was no significant statistical difference in change in mean cervical lordosis ( 2 wk vs. 2 year post-op) between 
the two groups $(\mathrm{p}>0.05)$. Similary, there were no significant statistical differences in change in mean $\mathrm{C} 2-\mathrm{C} 7$ sagittal plumbline and $\mathrm{T} 1$ slope ( 2 wk vs. 2 year post-op) between the two groups $(p>0.05)$. Rate of pseudarthrosis was higher in group I (21.2\%) than group II $(10.96 \%)$. This differnce was statistically significant $(\mathrm{p}<0.05)$. There were significant improvements in mean clinical outcomes (i.e. visual analog scale and oswestry disability index) at 2 years follow ups in both groups but there were no statistically significant differences between the two groups $(\mathrm{p}>0.05)$.

Conclusion: Our analyses indicate that both groups had similar clinical and radiographic outcomes. Lower pseudarthrosis rate but higher EBL, OR and LOS in group II suggest that extension of posterior cervical fusions into the thoracic spine still remains debatable. Prospective studies with additional patients and greater statistical power are needed to elucidate optimal means of posterior stabilization in patients with degenerative cervical disease.

\section{3rd Place Resident Fellow Paper Award • \$500}

Presentation \#78. Should Asymptomatic Levels with MRI Abnormalities be Included in an ACDF Construct? A Long-Term MRI Analysis

Marcus D. Mazur, MD, Salt Lake City, UT; Andrew T. Dailey, MD, Salt Lake City, UT; Lubdha M. Shah, MD, Salt Lake City, UT; Joel D. MacDonald, MD, Murray UT

Introduction: ACDFs are generally limited to the levels that are causing neurologic symptoms. But there are situations where asymptomatic levels may be considered for inclusion in an anterior construct, such as if there is severe radiographic degeneration adjacent to symptomatic levels. We evaluated whether the presence of asymptomatic preoperative MRI abnormalities was predictive of reoperation for symptomatic adjacent segment degeneration (ASD) after ACDF.

Methods: We reviewed patients at our institution who underwent an ACDF between 2000 and 2010 and had MRIs both preoperatively and postoperatively at least 3 years after the index surgery to evaluate new neurologic symptoms. We intended to exclude patients who had inadequate treatment, residual disease, or early recurrence after the index ACDF. MRIs were scored for ASD severity using published criteria. Patients were stratified according to the ASD severity score. Logistic and Cox regression analyses were used to evaluate the association between preoperative MRI abnormalities and reoperation for ASD after adjusting for covariates.

Results: Of 2,246 patients who underwent an ACDF during the study period, $96(4 \%)$ had MRI evaluation at least 3 years postoperatively for new symptoms. Mean follow-up was 78 months. Of the 195 adjacent segments evaluated, 14 (7\%) underwent subsequent fusion procedures. The 10-year surgery-free survival estimate was $82.7 \%$ (73.4 - 93.2\%). After adjusting for covariates, preoperative MRI abnormalities were predictive of reoperation only for the group with the highest severity score [HR

$4.5(1.0-19.8)]$ and those with foraminal stenosis [HR 4.2 (1.412.7)] (Figure). However, the prevalence of reoperation for ASD in these groups was only $16 \%$ and $15 \%$, respectively.

Conclusions: The prevalence of reoperation for ASD is low for patients who present with new symptoms several years after the index ACDF. Our findings do not support including asymptomatic levels in an anterior fusion construct, even if severe MRI abnormalities are present. Presentation \#79. Thoracolumbar Reciprocal Changes following
Cervical Reconstruction Surgery for Cervical Kyphosis

Jun Mizutani, MD, PhD, Nagoya, Japan; Strom Russel, San Francisco, CA; Kenji Endo, Tokyo, Japan; Kuniyoshi Abumi, MD, Sapporo, Japan; Ken Ishii, MD, PhD, Tokyo, Japan; Mitsuru Yagi, MD, PhD, Tokyo, Japan; Bobby Tay, MD, San Francisco, CA; Vedat Deviren, MD, San Francisco, CA; Christopher P. Ames, MD, San Francisco, $C A$

Introduction: There is a complex interaction among each spinal segment. Reciprocal change in the cervical spine has been noted following thoracolumbar deformity surgery. However, little is known about the effect of cervical deformity surgery on thoracolumbar alignment. This study identifies changes in thoracolumbar alignment following cervical reconstruction surgery for cervical kyphotic deformity.

Materials and Methods: We conducted a retrospective multicenter study of adult patients undergoing cervical reconstruction surgery. 78 patients were identified after excluding those with coronal deformity $>30^{\circ}$ or prior thoracolumbar fusion. Sagittal radiographic parameters were measured before and after surgery. Preoperative cervical sagittal alignment was categorized as imbalanced (CSI, C2$\mathrm{C} 7 \mathrm{SVA} \geq 40 \mathrm{~mm}, \mathrm{~N}=56$ ) or balanced (CSB, C2-C7SVA $<40 \mathrm{~mm}, \mathrm{~N}$ $=16)$. Preoperative thoracolumbar sagittal balance was categorized as C7P $(C 7 S V A \geq 0, N=41)$ or $\mathrm{C} 7 \mathrm{~N}(\mathrm{C} 7 \mathrm{SVA}<0, \mathrm{~N}=31)$. Using paired t-test, the effect of cervical reconstruction on thoracolumbar alignment was analyzed for the entire cohort and each subgroup. A p value $<0.05$ was considered as statistically significant.

Results: In the entire cohort, cervical reconstruction surgery caused significant changes in TK, LL, and T1 slope from $33.5^{\circ}$ to $37.0^{\circ}(\mathrm{P}=$ $0.002),-51.0^{\circ}$ to $-48.5^{\circ}(\mathrm{P}=0.0135)$, and $20.8^{\circ}$ to $30.5^{\circ}(\mathrm{P}<0.0001)$, respectively. The $\mathrm{C} 7$ plumb line shifted significantly anteriorly (mean C7SVA from $-6.7 \mathrm{~mm}$ to $17.3 \mathrm{~mm}, \mathrm{P}=0.0003)$. C2-C7SVA and cervical kyphosis improved from $61.6 \mathrm{~mm}$ to $36.5 \mathrm{~mm}(\mathrm{P}<0.0001)$ and from $27.2^{\circ}$ to $-1.2^{\circ}(\mathrm{P}<0.0001)$, respectively. The CSI group had significant reciprocal change in several thoracolumbar parameters such as TK; from 36.2 to 39.2 ( $\mathrm{P}=0.00125)$, LL; from-52.1 to -48.4 $(\mathrm{P}=0.0063), \mathrm{LL}(4-\mathrm{S})$; from -30.8 to $-27.9(\mathrm{P}=0.0148)$, C7SVA; from -25.3 to $9.7(\mathrm{P}=0.0002)$, and T1slope; from 23.0 to $31.0(\mathrm{P}=$ $0.0013)$. However, only the T1 slope changed significantly from 17.2 to $26.4 \mathrm{P}=0.0142$ in the $\mathrm{CSB}$ group. The $\mathrm{C} 7 \mathrm{~N}$ group experienced significant changes in TK; from 29.9 to $33.9(\mathrm{P}=0.0005)$, TK(8-12); from 10.4 to $12.4(\mathrm{P}=0.0421)$, $\mathrm{LL}$; from -56.6 to $-51.2(\mathrm{P}=0.0028)$, T1 slope; from 15.5 to 27.3 ( $\mathrm{P}<0.0001)$, and PI-LL from-1.3 to 5.7 ( $\mathrm{P}$ $=0.0003)$. On the other hand, there were no significant thoracolumbar and PI-LL changes in the C7P-group. In terms of cervical sagittal parameters, such as, C2-C7SVA was decreased less than $40 \mathrm{~mm}$ in both $\mathrm{C} 7 \mathrm{P}$-group and $\mathrm{C} 7 \mathrm{~N}$-group, indicating cervical reconstruction surgery improved cervical sagittal imbalance. COG-SVA, which is the indicator of head position, was decreased from $129.6 \mathrm{~mm}$ to $78.9 \mathrm{~mm}$ $(\mathrm{P}=0.0052)$ in C7P-group, on the contrary, COG-SVA was increased from $32.1 \mathrm{~mm}$ to $40.0 \mathrm{~mm}(\mathrm{P}=0.0361)$ in $\mathrm{C} 7 \mathrm{~N}$-group. Also, The $\mathrm{C} 7 \mathrm{~N}-$ group experienced significant changes in C7SVA; from -51.2 to -3.47 $(\mathrm{P}<0.0001)$, however, there was no significant in C7P-group; from 45.2 to 44.0 .

Conclusion: This is the first report of thoraco-lumbar reciprocal change following cervical reconstruction surgery. The reciprocal change was dependent on preoperative cervical and thoracolumbar sagittal balance. Cervical reconstruction surgery can restore both cervical sagittal alignment and global spinal harmony. 
Presentation \#80. Preoperative Global Sagittal Imbalance is a Predictor of Postoperative Neck Pain following Laminoplasty in Patients with Cervical Spondylotic Myelopathy: Based on the Prospective Analysis of 165 Patients

Jun Ouchida, MD, Nagoya, Japan; Hiroaki Nakashima, MD, Nagoya, Japan; Naoki Segi, MD, Konan, Japan

Introduction: Cervical laminoplasty is an established procedure in the treatment of cervical spondylotic myelopathy (CSM). Although favorite outcome have been reported, postoperative neck pain and persistent extremity pain are known to be a problem. While some reports indicated the influence of regional cervical alignment on surgical outcomes, few reports investigated the correlation between postoperative neck / extremity pain and global sagittal balance. The purpose of this study was to investigate an influence of global sagittal balance to clinical outcomes after cervical laminoplasty.

Materials and Methods: A hundred and sixty-five patients with cervical spondylotic myelopathy were prospectively enrolled. Whole-spine radiographs were obtained preoperatively in the standing position. Patients were stratified into three groups [group 0; sagittal vertical modifier (SVA) $<4 \mathrm{~cm}$, group +: SVA 4 to $9.5 \mathrm{~cm}$, group ++ : SVA $>9.5 \mathrm{~cm}$ ) by C7-SVA according to Scoliosis Research Society (SRS)-Schwab Adult Spinal Deformity (ASD) Modifier. We also evaluated clinical outcomes on the Japanese Orthopaedic Society (JOA) score, neck pain and extremity pain (upper / lower) on the visual analogue scale (VAS) before and one year after laminoplasty.

Results: After excluding 65 cases for a paucity of adequate radiographic examination, or an insufficiency of follow-up duration ( $>12$ months), a hundred patients (60 males and 40 females with an average age of 63.1 years) were evaluated. The mean preoperative C7-SVA was $5.2 \pm 6.7 \mathrm{~cm}$ (Group $0, \mathrm{~N}=45$; group,$+ \mathrm{N}=27$; group ,$++ \mathrm{N}=28$ ). The mean preoperative VAS for lower extremity pain were $2.2 \pm 2.7$ in group $0,4.4 \pm 3.8$ in group + , and $4.0 \pm 3.8$ in group ++ . The mean pre- and post- operative JOA score were $11.7 \pm 2.4$ and $14.7 \pm 2.3(\mathrm{p}<0.001)$ in group $0,10.9 \pm 3.0$ and $14.1 \pm 2.0(\mathrm{p}<0.001)$ in group + , and $10.2 \pm 2.5$ and $13.2 \pm 2.9(\mathrm{p}<0.001)$ in group ++ . With respect to neck pain, the mean pre- and post- operative VAS were $3.7 \pm$ 2.9 and $3.7 \pm 2.5$ in group $0,3.4 \pm 3.4$ and $4.1 \pm 2.7$ in group + ,

and $3.3 \pm 3.3$ and $5.0 \pm 2.7(\mathrm{p}<0.01)$ in group ++ .

Conclusions: Patients with mild or severe global sagittal imbalance (group,+++ ) revealed higher VAS in lower extremity preoperatively than patients with non pathologic sagittal balance (group 0). The mean JOA score in each groups improved significantly at one year following surgery. In this study, a postoperative deterioration of neck pain was seen in group ++ . While cervical laminoplasty is still effective for CSM patients with global sagittal imbalance, surgeons should keep in mind the possibility of a postoperative deterioration of physical complaints in treatment patients with severe global sagittal imbalance.

\section{Presentation \#81. Adult Spinal Deformity Patients with Proximal Junctional Kyphosis Adjust with Cervical Malalignment at Similar Rates but Distinct Characteristics Relative to those Unaffected}

Peter G. Passias, MD, Westbury, NY; Cyrus M. Jalai, BA, New York, NY; Han Jo Kim, MD, New York, NY; Justin S. Smith, MD, PhD, Charlottesville, VA; Christopher P. Ames, MD, San Francisco, CA; D. Kojo Hamilton, Pittsburgh, PA; Robert Eastlack, MD, San Diego, CA; Douglas C. Burton, MD, Kansas City, KS Munish Gupta, MD, St. Louis, MO Robert Shay Bess, MD, Rye, NY; Virginie Lafage, PhD, New York, NY; Frank Schwab, MD, New York, NY; International Spine Study Group, Brighton, CO

Introduction: Post-operative proximal junctional kyphosis (PJK) has been analyzed for associations with increased regional deformity, but cervical alignment is rarely considered in these patients. Specifically, the impact of PJK and the onset of cervical deformity is understudied, notably in the context of increasing PJK angle and global sagittal deformity worsening. This study aims to analyze cervical malalignment onset as a result of PJK following adult spinal deformity (ASD) corrective surgery.

Materials and Methods: Retrospective review of a prospective, multi-center ASD patient registry. Inclusion criteria: primary ASD patients ( $\geq 5$ levels fused, UIV at T7 or above, 1 year minimum follow-up) without baseline cervical deformity (CD). CD was defined as meeting $\geq 2$ of the following criteria on baseline radiographs: TS-CL $<20^{\circ}, \mathrm{cSVA}<4 \mathrm{~cm}, \mathrm{C} 2-\mathrm{C} 7 \mathrm{CL}>10^{\circ}$. PJK presence $\left(<10^{\circ}\right.$ change in UIV and UIV +2 kyphosis) and angle were identified at 1 year post-operative. SRS-Schwab classification modifier (PI-LL, SVA, PT) grades were also assigned at baseline and lyear $\mathrm{f} / \mathrm{u}$. ANOVA and t-tests compared radiographic parameters across and within PJK groups.

Results: Of 193 patients showing baseline radiographic cervical alignment, PJK developed in $69(35.8 \%)$ patients, with 34 (17.6\%) instances occurring at / above T7. PJK patients had significantly greater CL and TS-CL 6w-1year change $(\mathrm{p}<0.018)$, and also displayed higher 1 year T1 Slope, CL, cSVA, and C2-T3 angle $(\mathrm{p}<$ 0.05 ). At 1 year post-op, the concomitant CD rate was $23.5 \%$. PJK angle (range: $10^{\circ}-48^{\circ}$ ) was stratified: $10^{\circ}-20^{\circ}(55.9 \%), 20^{\circ}-30^{\circ}$ $(23.5 \%),>30^{\circ}(20.6 \%)$. The highest angle group corresponded to significantly increased T1 Slope, CL, cSVA, and C2-T3 angle ( $\mathrm{p}<$ 0.05 all) in PJK patients. Global malalignment (SVA) also increased at 1 year with increasing PJK angle $(\mathrm{p}=0.040)$. The prevalence of higher PI-LL and SVA modifier grades at baseline was significantly higher in PJK patients $(\mathrm{p}<0.001)$ though not at 1 year follow-up. PJK patients with high $(+/++) 1$ year SVA modifiers displayed higher T1 Slope and TS-CL at 1 year; PJK patients with higher $(+/++) 1$ year PI-LL modifiers also showed higher T1-CL and C0 Slope $(\mathrm{p}<0.05$, all). PJK patients with high PT modifier grades did not significantly differ in cervical alignment.

Conclusions: ASD patients adjust for PJK with cervical malalignment onset with increased C2-T3 angle and CL onset at 1year. Global sagittal deformity and increasing PJK angle were both related to increased cervical deformity. Considerations for the cervical spine in PJK patients should be taken into account.

Presentation \#82. The Difference of Spinal Sagittal Alignment and Health-Related QoL Between Males and Females with Cervical Deformity

Shin Oe, MD, Hamamatsu, Japan; Daisuke Togawa, MD, PhD, Hamamatsu, Japan; Tomohiko Hasegawa, MD, PhD, Shizuoka, Japan; Yu Yamato, MD, PhD, Hamamatsu, Japan; Sho Kobayashi, MD, PhD, Hamamatsu, Japan; Tomohiro Banno, MD, Shizuoka, Japan; Yuuki Mihara, MD, Hamamatsu, Japan; Kenta Kurosu, MD, Hamamatsu, Japan; Yukihiro Matsuyama, MD, PhD, Hamamatsu, Japan

Introduction: There are some reports that adult spinal deformity, especially in lumbosacral lesion, is frequently observed in females. However, there are also some reports recently that the factors which HRQOL deteriorate are not only lumbar spine and pelvic malalignment but also cervical deformity (CD). There are some reports that cervical deformity is observed more in males. However, there are few studies that investigate the difference of mechanism of spinal deformity between males and females. The purpose of this study were to clarify separately in gender the spinal sagittal alignment and HRQOL in health screening volunteers aged over 50 with $\mathrm{CD}$.

Methods: This cohort study included 656 volunteers aged 50 to 89 years (263 males and 393 females, mean age 73). The definition of CD was C2-7 sagittal vertical axis (SVA) over $40^{\circ}$. The volunteers were divided into 4 groups (CDM: males with $\mathrm{CD}$, NCDM; males without $\mathrm{CD}, \mathrm{CDF}$; females with $\mathrm{CD}, \mathrm{NCDF}$ : females without $\mathrm{CD}$ ). Whole spine $\mathrm{X}$-rays were taken in standing position for all volunteers. Pelvic 
tilt (PT), Lumbar lordosis (LL), pelvic incidence minus LL (PI-LL), Thoracic kyphosis (TK), T1 slope (T1S), cervical lordosis (CL), T1SCL, C2-7 sagittal vertical axis (SVA), C7 SVA were measured using software (Surgimap SPINE). HRQOL was evaluated by EQ-5D.

Results: The numbers of each group were 82 in Group CDM, 181 in Group NCDM, 36 in CDF, 357 in Group NCDF. The average parameters in each groups (CDM, NCDM, CDF, and NCDF) was PT (15, 14, 26, and 21 degrees), PI-LL (7, 5, 16, and 10 degrees), C2-7 SVA (49, 24, 46, and 20mm), C7 SVA (61, 40, 75, and 47mm), EQ-5D $(0.82,0.88,0.78$, and 0.81), respectively. In females, PT was significantly greater in Group CDF compared to Group NCDF $(\mathrm{P}<0.05)$. However, there were no significant difference in pelvic parameters between group CDM and NCDM in males. In comparison between $\mathrm{CDM}$ and $\mathrm{CDF}$, there were no significant difference in cervical parameters, but only PT in CDF was significantly higher than that in CDM $(\mathrm{P}<0.01)$. Moreover, $\mathrm{CDM}$ had significantly deteriorated EQ-5D compared to NCDM $(\mathrm{P}<0.05)$.

Conclusions: Different mechanism of sagittal spinal deformity was observed between males and females in the present study. Group CDF had deteriorated PT and PI-LL compared to group NCDF. However those in group CDM were not significantly different compared to those of group NCDM. Group CDF had already deterioration of spinopelvic alignment, although it was kept well in group CDM. This means that the deterioration of spinal sagittal alignment in male originate from cervical spine. Moreover, EQ-5D in group CDM was significantly deteriorated than that in group NCDM, while deterioration of lumbopelvic parameters had less influenced in males $(\mathrm{P}<0.05)$. This result suggested that $C D$ was associated with HRQOL.

\section{Presentation \#83. Analysis of Successful vs. Failed Radiographic Outcomes following Cervical Deformity Surgery}

Themistocles S. Protopsaltis, MD, New York, NY; Subaraman Ramchandran, MD, New York, NY; D. Kojo Hamilton, MD, Pittsburgh, PA; Daniel Sciubba, MD, Baltimore, MD; Peter G. Passias, MD, Westbury, NY; Virginie Lafage, PhD, New York, NY; Renaud Lafage, MS, New York, NY; Justin S. Smith, MD, PhD, Charlottesville, VA; Robert A. Hart, MD, Portland, OR; Munish Gupta, MD, St. Louis, MO; Doug Burton, MD, Kansas City, KS; Robert Shay Bess, MD, Rye, NY; Christopher I. Shaffrey, MD, Charlottesville, VA; Christopher P. Ames, MD, San Francisco, CA; International Spine Study Group, Brighton, $\mathrm{CO}$

Introduction: Recent studies have demonstrated correlation between cervical sagittal alignment and patient reported outcomes. Few studies have explored cervical deformity correction prospectively and the factors that result in successful vs. failed cervical alignment corrections remain unclear. The purpose of this study was to evaluate pre-operative alignment and surgical factors associated with suboptimal early post-operative radiographic outcomes following surgery for cervical deformity.

Methods: Adult cervical deformity (CD) patients were consecutively enrolled in a multi-center database. Inclusion criteria were cervical kyphosis $>10^{\circ}$, cSVA $>4 \mathrm{~cm}$, or CBVA $>25^{\circ}$. Patients were categorized into failed outcomes group if cSVA $>$ $4 \mathrm{~cm}$ or T1Slope-Cervical Lordosis (TS-CL) $>20^{\circ}$ at 6 months post operatively. Demographic, surgical and pre-operative radiographic measures were compared between failed and successful deformity corrections. Multivariate analysis using binary logistic regression was performed to evaluate for associations between radiographic parameters and failed outcomes with respect to cSVA and TS-CL separately.

Results: $71 \mathrm{CD}$ patients (mean age 62 yrs, 56\% female, $41 \%$ revisions, minimum 6 mos. follow up) were included. 45 had primary deformities within the cervical spine and 26 at the CT junction. Overall $33(46.4 \%)$ had failed radiographic outcomes by cSVA and 46 (64.7\%) by TS-CL. Failure to restore cSVA was associated with worse preop
C2-pelvic tilt angle (CPT: 64.4 vs $47.8^{\circ}, \mathrm{p}=.01$ ), presence of any "+ "Schwab modifier $(\mathrm{p}=.007)$, revision surgery $(\mathrm{p}=.05)$ and failure to address the secondary, thoracolumbar driver of the deformity $(\mathrm{p}=$ $.02)$. Patients with failed corrections of cSVA had worse postoperative C2Slope (35.0 vs $\left.23.8^{\circ}, \mathrm{p}=.004\right)$, TS-CL (35.2 vs $24.9^{\circ}, \mathrm{p}=.01$ ), and CPT $\left(47.9\right.$ vs $\left.28.2^{\circ}, \mathrm{p}<.001\right)$. Failure to correct TS-CL was associated with worse preoperative cervical kyphosis $(10.4$ vs

$-2.1^{\circ}, \mathrm{p}=.03$ ), and CPT (52.6 vs $\left.39.1^{\circ}, \mathrm{p}=.04\right)$. Patients with failed corrections of TS-CL had worse postoperative C2Slope (30.2 vs $\left.13.3^{\circ}, \mathrm{p}<.001\right)$, cervical lordosis $\left(-3.6\right.$ vs $\left.-15.1^{\circ}, \mathrm{p}=.01\right)$, and CPT (37.7 vs $\left.24.0^{\circ}, \mathrm{p}<.001\right)$. Multivariate analysis revealed occurrence of post-operative DJK (kyphosis $>10^{\circ}$ at LIV to LIV-2 from pre- to post-op) as the only significant parameter associated with sub-optimal outcomes with respect to cSVA (OR- 0.06, CI- 0.01-0.4, $\mathrm{p}=.004$, Figure 1) and TS-CL (OR-0.15, CI- 0.02-0.97, $\mathrm{p}=.05$ ).

Conclusions: Surgery to correct CD can be challenging. Factors that were associated with failure to correct the cSVA included revision surgery, worse preop CPT, concurrent thoracolumbar deformity, and failure to correct secondary, thoracolumbar deformity drivers. Failure to correct the TS-CL mismatch was associated with worse preoperative cervical kyphosis and CPT. Occurrence of early post-operative DJK significantly affects post-operative radiographic outcomes.

\section{Presentation \#84. Laminoplasty Decreases Postoperative Axial Neck Pain Scores in Myelopathic Patients: A Comparsion with Laminectomy and Fusion}

John M. Rhee, MD, Atlanta, GA; Thomas M. Neustein, BA, Atlanta, GA; Salvador Rafael Arceo V, Atlanta, GA

Introduction: Postoperative new or worsening axial neck pain is commonly cited as a major disadvantage of laminoplasty. However, there remains a paucity of corroborative data from large series. In this study, we examined axial pain and other clinical outcomes after laminoplasty (LP) in a large cohort of myelopathic patients and compared them to a cohort undergoing laminectomy and fusion (LF).

Materials and Methods: Following IRB approval, we reviewed the medical records, radiographs, and prospective clinical outcomes database of 85 patients undergoing LP and 52 patients undergoing LF for cervical myelopathy with minimum 1-year radiographic follow-up and average clinical follow-up of 18.5 months. Primary outcomes included Visual Analogue Scale neck pain score (VAS, average and worst), neck disability index (NDI) score, patientreported pain location, and SF-36 Mental / Physical Component Scores. Secondary outcomes included Modified Japanese Orthopaedic Association (mJOA) scores and radiographic parameters (C2-7 Cobb and T1 Slope).

Results: Preoperatively, there were no significant differences between the groups with respect to age, mJOA, SF-36, VAS neck pain, NDI, Miyazaki spondylosis score, AP spinal cord dimension, and T1 slope. However the LP patients had greater preoperative lordosis (C2-7 Cobb: LP 12.69o \pm 10.40; LF $3.960 \pm 13.39$, p < 0.0001). VAS-worst (Figure 1a) significantly improved for LP $(-1.7 \pm 0.55, \mathrm{p}=0.03)$ and trended to improvement for LF $(-1.0 \pm 0.59, \mathrm{p}=0.09)$. VAS-average (Figure 1b) significantly improved in both groups (LP $-1.4 \pm 0.51$, $\mathrm{p}=0.008 ; \mathrm{LF}-1.04 \pm 0.52, \mathrm{p}=0.05$ ). NDI (Figure 2) significantly improved for the LP group $(-6.79 \pm 2.25, \mathrm{p}=0.0032)$ but not for LF $(-4.01 \pm 3.05, \mathrm{p}=0.19)$. mJOA scores significantly improved in both groups $(\mathrm{LP}+2.89 \pm 0.27, \mathrm{p}<0.0001 ; \mathrm{LF}+2.45 \pm 0.33, \mathrm{p}<0.0001)$. SF-36 MCS and PCS scores did not significantly change in either group. There was a small but statistically significant loss of lordosis in the LP group (-2.92 degrees, $\mathrm{p}=0.0181)$; no significant change was noted in the LF group $(-1.25$ degrees, $\mathrm{p}=0.53)$.

Conclusion: In one of the largest case-control series of patients undergoing laminoplasty for cervical myelopathy, LP led to significant improvement in axial neck pain similar to that seen with LF. Neck pain improved rather than worsened after both operations. Similar 
significant improvements in mJOA occurred in both groups, but NDI improved significantly only for LP. In the appropriately selected patient, LP can be performed to treat myelopathy despite the presence of axial neck pain with an expectation that it may actually improve rather than worsen.

Presentation \#85. The Pa-mJOA: A Patient-Derived, SelfReported Outcome Instrument for Measuring Myelopathy Comparison with the mJOA

John M. Rhee, MD, Atlanta, GA; Weilong Jeffrey Shi, MD, Atlanta, GA; Jin Young Kim, MD, Atlanta, GA; Feifei Zhou, MD, Beijing, China; Anuj Patel, MD, Atlanta, GA

Introduction: Although the mJOA is widely used in the assessment of cervical myelopathy, one downside is that it is not a patient-derived outcome. If available, a patient-derived mJOA (Pa-mJOA) might more accurately access the burden of myelopathy by removing physician biases. Furthermore, a Pa-mJOA could facilitate research because the data would be immediately available instead of requiring the researcher to complete the instrument retrospectively. The JOA Cervical Myelopathy Evaluation Questionnaire (JOACMEQ) is a patient-derived instrument to assess myelopathy, but it is long and has not correlated well with the mJOA. The purpose of this study is to evaluate a patient derived, self reported version of the mJOA (the Pa-mJOA) that a patient can complete along with other patientderived outcome measures.

Materials and Methods: The Pa-mJOA was created by slightly modifying / expanding upon the verbiage of the mJOA to make it possible for a patient to understand and complete the instrument while not changing the core structure of the questionnaire (TABLE 1). 100 consecutive consenting patients (both pre and postoperative) with cervical myelopathy presenting to a spine clinic completed the survey over a 4-month period. After the patient completed the Pa-mJOA, the mJOA was scored by a physician blinded to the Pa-mJOA result. The results of the Pa-mJOA were compared to that of the mJOA, and statistical analysis performed.

Results: Mean Pa-mJOA score (14.68) was almost identical to mean mJOA score $(14.66)(\mathrm{p}=0.89)$. The overall kappa coefficient for Pa-mJOA was 0.66 , which suggests substantial agreement with the mJOA. The Cronbach's alpha was 0.62 for the Pa-mJOA and 0.65 for the mJOA, suggesting very similar internal consistency for both instruments in measuring myelopathy. There were no significant differences in $\mathrm{Pa}$ - mJOA and mJOA scores for those with mild and moderate myelopathy (Table 2). There was a statistically significant difference in those with severe myelopathy, but the number of severe patients in this study was small $(\mathrm{n}=13)$, and the difference was 1.1, which falls below the MCID of 3 quoted in the literature for severe myelopathy. When asked how the survey should be administered, $67 \%$ of patients preferred (strongly to slightly) to fill out the Pa-mJOA themselves rather than having the physician complete it for them, suggesting low patient burden for completing the survey. Only $17 \%$ strongly preferred the physician to complete it.

Conclusions: The Pa-mJOA provided very similar scores to the mJOA in assessing myelopathy. The Pa-mJOA shows promise as a patient-derived outcome that can readily be completed by the patient with results similar to those obtained using the mJOA. Comprising the same 5 questions as the mJOA but reworded for ease of patient comprehension, the Pa-mJOA also demonstrated low patient burden in completing the survey. Further validation is necessary, especially to determine its responsiveness to changes in myelopathy, as well as in those with more severe myelopathy.
Presentation \#86. Accuracy of Post-Operative Recall of Baseline Neurological Function by Patients Undergoing Surgical Decompression for Cervical Spondylotic Myelopathy

Nanfang Xu, New York, NY; Shaobo Wang, MD, Beijing, China

Introduction: Patient satisfaction over decompressive surgery for cervical spondylotic myelopathy (CSM) is affected by their perceived change in neurological function. The Japanese Orthopaedic Association (JOA) scale is one of the primary measurement tools for neurological function in these patients. Instead of comparing pre- and post-operative JOA scores, this perceived change is really determined by comparing their self-recalled pre-operative status against how they feel at the follow-up visit. In this study we aim to examine the accuracy of patient recall of their baseline neurological function when compared with their pre-operative scores.

Material and Methods: CSM Patients who underwent decompressive surgery at a single institution between 2008 and 2010 were identified and those with at least three JOA scores (baseline, recall, and current) were included. The Wilcoxon signed-rank test was used to evaluate the mean difference between recall and baseline. A generalized estimating equation regression model was built to identify predictors for recall error. Included predictors were baseline JOA, JOA improvement rate, gender, age, follow-up time, and the type of procedure. Bivariate analysis was first performed to evaluate the impact of each single predictor. A backward algorithm was then used to determine their significance, and the quasi-likelihood information criterion was applied in cases of colinearity. The SAS software (version 9.4) was used for statistical analysis.

Results: 77 patients were included in the final analysis. The gender ratio was 6:4. Mean age was 59 (range: 29 - 77) years. Follow-up was available at 3,12 and 24 months. Overall, there was no significant difference between baseline and recall JOA scores regardless of follow-up time. Lower baseline JOA, higher improvement rate, and the female sex were determined as significant predictors for greater recall error by the final multivariate analysis model.

Conclusion: Post-operative patient satisfaction is associated with the self-perceived neurological improvement, and it is important to see if patients could accurately recall their pre-operative functional status. Patient recall of their baseline neurological function was accurate regardless of the length of follow-up time. For patients in a certain visit, higher recall accuracy was associated with lower baseline JOA, higher improvement rate, and the female sex.

Presentation \#87. What are the Research Priorities for Patients with Degenerative Cervical Myelopathy?

Mark R. Kotter, MD, PhD, Cambridge, United Kingdom; Davies M. Benjamin, MBChB, MRCSEd, Cambridge, United Kingdom

Introduction: Cervical Spondylotic Myelopathy [CSM] is a common cause of spinal dysfunction and despite optimal therapy, many live with significant disabilities. Misalignment of patient and clinician objectives is felt to contribute to research wastage. In North America, in $2010,85 \%$ of biomedical research was felt to have yielded no actual or potential clinical benefit. Our objective therefore was to assess the research priorities of patients with degenerative cervical myelopathy to meet the needs of our patients.

Design: Cross-sectional online patient survey.

Subjects: Patients with self-reported CSM.

Methods: CSM patients, registered with the non-profit organisation myelopathy.org, were invited to complete an online survey, to rank the 7 functional domains of spinal cord injury in order of priority. First choice domains were weighted with 7 points and the least preferred option with 1. Average scores were calculated. Patient demographics and current disability (mJOA) was also noted.

Results: 106 patients $(\mathrm{M}=31, \mathrm{~F}=75)$, average age $55 \pm 11$ years completed the survey. Priorities in rank order were elimination of pain 
(5.6), recovery of walking (5.3), arm / hand (5.2), upper body strength / balance (3.4), bladder / bowel function (3.3), normal sensation (3.2) and sexual function (2.2). Age, sex and prior surgery did not influence priorities. Patients with severe myelopathy $(\mathrm{mJOA}<12)$ prioritised recovery of hand function over pain.

Conclusions: Alleviation of pain, and improvements in mobility and hand function emerge as key priorities for patients and should be a focus for researchers and outcome measures in clinical trials for CSM.

Presentation \#88. The Impact of Cervical Sagittal Alignment on Axial Neck Pain and Health-Related QOL after Laminoplasty: A Prospective Comparative Study between Cervical OPLL and CSM

Hiroyasu Fujiwara, MD, Kawachinagano, Osaka, Japan; Take Oda, MD, PhD, Kawachinagano, Osaka, Japan; Takahiro Makino, MD, DMSC, Suita, Osaka, Japan; Yu Moriguchi, MD, PhD, Suita, Osaka, Japan; Kazuo Yonenobu, MD, DMSC, Osaka, Japan; Takashi Kaito, $M D$, PhD, Osaka, Japan

Introduction: Many studies have focused on postoperative axial neck pain after laminoplasty. However, there exist only a few reports that investigated the correlation among axial neck pain, patientbased QOL outcome measure; JOACMEQ (Japanese Orthopaedic Association Cervical Myelopathy Evaluation Questionnaire), and cervical sagittal alignment. The purpose of this study is to prospectively investigate the correlation among axial neck pain, JOACMEQ, and cervical sagittal alignment after laminoplasty for cervical myelopathy on the hypothesis that cervical sagittal malalignment has negative effects on axial neck pain and JOACMEQ.

Materials and Methods: Consecutive fifty-seven patients who treated by open-door laminoplasty for cervical myelopathy were included (mean age: 63.7 years, 15 females and 42 males), and divided into two groups by the diagnosis (CSM group: 35 patients, OPLL group: 22 patients). JOA score, JOACMEQ, 10-second test, and VAS for axial neck pain (VAS) were assessed at the time points of before surgery and postoperative 12 months (POM12). Radiographic parameters were measured by $\mathrm{C} 2$ sagittal vertical axis (C2 SVA), C2-C7 lordosis, T1 sagittal slope, and cervical sagittal range of motion on flexion and extension (ROM). The Spearman's rank-correlation coefficient was used for the statistical analysis, and the significance was set by $\mathrm{R}>0.4$ and $\mathrm{P}<0.05$.

Results: The value of C2 SVA in both groups have slightly shifted to anterior from the respective pre-op. value (CSM: $+19.7 \pm 10.9$ mm, OPLL: $+22.1 \pm 13.4 \mathrm{~mm}$ ) to the value at POM12 (CSM: + $23.2 \pm 16.1 \mathrm{~mm}$, OPLL: $+28.7 \pm 15.4 \mathrm{~mm}$ ). The postoperative VAS in OPLL group showed strong negative correlations with C2 SVA and T1 sagittal slope (Table 1). And, the strong negative correlations were found between the VAS and cervical spine function in both preoperative CSM and OPLL group (CSM; $\mathrm{R}=-0.45, \mathrm{P}=0.01$, OPLL; $\mathrm{R}=-0.61, \mathrm{P}<0.01)$ and between the VAS and cervical spine function in postoperative OPLL group $(\mathrm{R}=-0.51, \mathrm{p}=0.05)$ (Table 2$)$.

Conclusion: This study demonstrated that significant negative correlation was found between the VAS and the subdomain of cervical spine function in JOACMEQ in preoperative CSM, OPLL and postoperative OPLL groups. However, radiographic cervical sagittal parameters were not significantly correlated with the VAS. Because the global sagittal alignments were not investigated in this study, the compensation by thoracic / lumber spine and lower extremities might affect the results. Further studies are underway including the relationship between axial neck pain and global sagittal alignment.
Presentation \#89. Complications and Readmission after Cervical Spine Surgery in Elderly Patients: An Analysis of 1,586 Patients

Ahmed Saleh, MD, Brooklyn, NY; Caroline Thirukumaran, MBBS, MHA, Rochester, NY; Robert W. Molinari, MD, Pittsford, NY; Addisu Mesfin, MD, Rochester, NY

Introduction: There is a paucity of literature describing risk factors for adverse outcomes after geriatric cervical spinal surgery. How safe is cervical spine surgery in elderly patients? Does patient selection, type of surgery, length of surgery, and other elderly patient comorbidities affect complication and readmission rates after surgery?

Methods: A retrospective cohort study was performed using data from the American College of Surgeons National Surgical Quality Improvement Program (ACS-NSQIP) database. Patients over the age of 65 who underwent cervical spinal surgery from 2005 2013 were identified using ICD-9 diagnosis codes and CPT codes. Outcome data was classified as either a major complication, minor complication, readmission, or mortality. Major complications included sepsis, pulmonary embolism, deep surgical site infection, organ or surgical site infection, unplanned intubation, CVA, MI, cardiac arrest, ventilator use $>48$ hours, septic shock, acute renal failure, peripheral nerve injury, coma, or graft / prosthesis / flap failure. Minor complications included blood transfusion, UTI, DVT, superficial SSI, pneumonia, renal insufficiency, and wound dehiscence. Multivariate logistic regression models were used to determine factors which placed the patients at risk to develop adverse outcomes in the initial 30 postoperative days.

Results: 1,586 patients over the age of 65 who underwent cervical spine surgery were identified. Overall, $150(9.46 \%)$ patients experienced at least one complication or death in the intial 30 postoperative days. $62(3.91 \%)$ patients experienced a major complication. $120(7.57 \%)$ patients experienced a minor complication. $71(5.99 \%)$ patients were readmitted to the hospital within 30 days. $13(0.82 \%)$ deaths recorded in the initial 30 postoperative days. Patients who were over the age of 75 were at higher risk of developing a complication than patients aged $65-70$, or $70-75$ (age $>75$, OR: 1.72 [1.09-2.71]. Increased operative times were also strongly associated with perioperative complications (operative time > 180 mins, OR: 3.49 [2.16 - 5.64]). Patients who had a baseline functional status of partially or totally dependent were also at higher risk for developing complications (Partially / Totally dependent, OR: 3.28 [1.83 - 5.88]). Other factors associated with increased complication rates were emergency cases and patients with and ASA classification of 3 or greater (Emergency, OR: 4.56 [1.66 - 12.54], ASA 3 / 4 / 5, OR: 1.77 [1.10 - 2.83]. Patients with preoperative pulmonary or nutritional / endocrine comorbidities were also at increased risk for developing a postoperative complication in the initial 30 postoperative days (Pulmonary, OR: 1.86 [1.19-2.92], Nutrition / Endocrine, OR: 2.07 [1.04-4.14]. Patients who developed at least one postoperative complication were at increased risk of readmission to the hospital within 30 days (Post-Op Complication, OR: 10.03 [5.07-19.84]).

Conclusions: Elderly patients undergoing cervical spinal surgery have a complication rate of $9.46 \%$ and readmission rate of $5.99 \%$ in the initial 30 postoperative days. Risk factors for complications include age greater than 75 , longer operative time, decreased baseline functional status, emergency cases, and higher ASA Classification. Patients with preoperative pulmonary or endocrine comorbidities were also at higher risk of postoperative complications. Higher readmission rates are associated with patients who experienced at least one postoperative complication. These risk factors should be considered when planning cervical spinal surgery in elderly patients. 


\section{Presentation \#90. Opioid Use Trends Following Cervical Spine Surgery}

Andrew J. Pugely, MD, Iowa City, IA; Nicholas A. Bedard, MD, Iowa City, IA; Jamal N. Shillingford, MD, New York, NY; Comron S. Saifi, MD, New York, NY; Joseph L. Laratta, MD, New York, NY; K. Daniel Riew, MD, New York, NY; Ronald A. Lehman, MD, New York, NY

Introduction: The United States is in the midst of an opioid epidemic. The purpose of this study purpose was to evaluate perioperative opioid use following cervical spine surgery comparing preoperative opioid users (OU) and non-opioid users (NOU).

Materials and Methods: The Humana Inc. dataset was reviewed from 2007 - 2015 for patients undergoing anterior or posterior cervical spine fusion surgery. Patients were identified using ICD-9 / CPT codes and prescription opioid use was measured by monthly prescription fill rates. An OU user was defined as opioid prescription within 3 months prior to surgery. Rates of opioid use were evaluated pre- operatively for OU and trended for one year post-operatively for both OU and NOU.

Results: In total, 14.801 procedures were evaluated, consisting of 12,921 patients with an anterior cervical (87.3\%), and $12.7 \%$ with a posterior cervical fusion. Overall, $51.4 \%$ of patients were preoperative OU. $47.7 \%$ of all NOU filled opioid prescriptions during their first post-operative month with less than $7.8 \%$ filling prescription by 3 -months for all NOU and re-fill rates ranged from $5.7-6.6 \%$ from $6-12$ months post-operatively. OU, however, had fill rates in the first month of $82.0 \%$, and a $6-12$ month fill rate between $44.9 \%$ and $46.9 \%$. NOU filled significantly less opioid prescriptions than OU at all time points $(\mathrm{p}<0.001)$. The fill rates did not differ significantly between anterior and posterior fusion surgery.

Conclusion: Approximately half of cervical spine fusion patients use opioids prior to surgery. Post- operative opioid use fell dramatically during the first 3 months in NOU, but nearly half of the pre-op opioid users will remain on narcotics at 1 year post-op in those using opioids before surgery. This data will serve as an important baseline to encourage discontinuation of opioids prior to spinal surgery.

\section{Presentation \#91. Adjacent-Level Degeneration after Bryan Cervical Disc Arthroplasty Compared with Anterior Discectomy and Fusion}

Justin W. Miller, MD, Carmel, IN; Rick C. Sasso, MD, Carmel, IN; Paul A. Anderson, MD, Madison, WI; K. Daniel Riew, MD, New York, $N Y$

Introduction: Anterior cervical discectomy and fusion (ACDF) is a reliable and proven procedure for the treatment of radiculopathy and / or myelopathy. Despite a successful track record for treating cervical disease, several potential limitations specific to ACDF including adjacent segment degeneration / disease, loss of viscoelastic disc properties, perioperative immobilization, bone graft site morbidity, pseudarthrosis, and plating complications have been identified. In an effort to improve upon the treatment of cervical disorders and avoid potential negative effects of a fusion, total disc arthroplasty (TDA) was developed. One of the original driving forces and theoretical basis for TDA was prevention of adjacent level degeneration. The purpose of this study is to compare adjacent level degeneration in both of these treatment groups using patients that were enrolled in a randomized controlled study. We also wish to assess the reliability of our measurements utilizing a method not previously described in the literature.

Materials and Methods: A total of 79 patients were enrolled and followed prospectively at two centers involved in a multicenter, FDA IDE trial for the BRYAN Cervical Disc arthroplasty. Neutral lateral radiographs were obtained preoperatively and postoperatively, and at 1, 2, 4, and out to 7 year follow-up after surgery. Inclusion criteria for analysis required images of sufficient clarity and ability to visualize the adjacent level above the study level. All original plain film images were digitized and the surgical level hidden for blinding purposes. The cephalad, adjacent level above the blinded procedure level was analyzed for all patients and time points by measuring the anteroposterior (AP) distance and the vertical disc height at the midpoint of the AP distance. A ratio was then created using disc height / AP distance in order to effectively compare images. Repeated measures ANOVA was used for data analysis.

Results: A total of 70 patients (Bryan Cervical Disc $\mathrm{N}=34$ and ACDF $\mathrm{N}=36$ ) met inclusion criteria and were included in this study. Changes over time were assessed for each group and between groups. Both the fusion and arthroplasty group showed a decrease in disc height over time that was significant $(\mathrm{p}=0.001)$ regardless of study group, indicative of adjacent level degeneration. Overall change in disc height between groups however, was not significantly different at any time point. Using our measurement technique, the overall inter-reviewer reliability was good (ICC $[95 \% \mathrm{CI}]=0.77[0.55,0.85]$ and intra- reviewer reliability was excellent $(0.93[0.91,0.94]$ and $0.85[0.81,0.87])$.

Conclusion: According to our data analysis, adjacent level degeneration occurs in a similar fashion in both the ACDF and TDA group. Our measurement technique is reliable and to the best of our knowledge not previously reported in the literature. There continue to remain inconsistencies in the literature regarding ACDF and TDA outcomes. It is important that we continue to follow these patients in order to obtain long-term follow-up data. Hopefully over time, such data will better clarify whether TDA can improve upon any of the shortcomings associated with ACDF, and specifically adjacent level disease.

Presentation \#92. Incidence, Epidemiology, and Treatment Trends for Spinal Epidural Abscesses Involving the Cervical Spine - A Single Institution 10 Year Retrospective Analysis

Zachary Denham, Columbus, $\mathrm{OH}$; Antonino Bucca, Brecksville, $\mathrm{OH}$; James Darnley, Columbus, OH; Kari Stammen, ATC, Columbus, OH; Ryan Rauck, MD, New York, NY; Sohrab Virk, MD, Columbus, OH; Safdar Khan, MD, Columbus, $\mathrm{OH}$

Introduction: Spinal epidural abscesses (SEA) affecting the cervical spine are exceedingly rare and little is reported in the literature pertaining to their incidence, epidemiology, and treatment trends. The purpose of this study was to determine the etiology and epidemiology of bacterial SEA involving the cervical spine and their treatment trends.

Methods: The medical center's data warehouse was queried for patients with ICD-9 diagnosis code for intraspinal abscess (324.1) over a 10-year period from September 24, 2001 to September 24, 2011. Patients with cytopathologic or radiographic evidence of bacterial SEA in the cervical or cervicothoracic spinal levels were included. Patients were excluded if they were under the age of 18. Patient demographics, vertebral levels, signs and symptoms, risk factors, microorganisms, pre / post- treatment clinical status, and treatment (medical vs. surgical) were recorded. Clinical status was recorded according to the following stages: stage $1=$ pain at level of affected spine; stage $2=$ nerve root pain radiating from involved spinal area; stage $3=$ motor weakness, sensory deficit or bladder / bowel dysfunction; and stage 4 = paralysis. Patients were diagnosed with a bacterial SEA involving the cervical spine (C1-C7) by either CT, MRI, or intraoperatively and had all listed outcome measures to be included in the study.

Results: A total of 43 patients were diagnosed with a SEA involving the cervical spine and included in the study. Males made up $60 \%$ of the study population $(\mathrm{n}=26)$, and the average age was $55.1 \pm 12.1$ years (range $28-79$ ). Locations of the SEA involving the cervical spine included 36 that were cervical $(\mathrm{C} 1-\mathrm{C} 7)$ and 7 that were cervicothoracic (C1-T12). The average number of levels involved was $3.9 \pm 2.7$ (range $1-15$ ). Patients presented in Stage 1 $(33 \%)$, Stage $2(12 \%)$, Stage $3(37 \%)$ and Stage $4(19 \%)$. Presenting signs and symptoms included elevated erythrocyte sedimentation rate $(60 \%)$, elevated white blood cell count $(81 \%)$, and fever (49\%). Risk 
factors included tobacco use (56\%), concurrent non-spinal infection $(37 \%)$, and diabetes mellitus $(35 \%)$. The most common grown organisms were methicillin-resistant Staphylococcus aureus (44\%) and methicillin-susceptible Staphylococcus aureus (23\%). Fifteen patients were treated with antibiotics only $(35 \%)$, and 28 were treated with both surgery and antibiotics (65\%).

Conclusion: Spinal epidural abscesses involving the cervical spine are challenging to treat and manage. The majority of patients presented with motor weakness / sensory deficit or neck pain. Tobacco use, nonspinal infections, and diabetes mellitus were found to be the biggest risk factors for spinal epidural abscesses of the cervical spine. Outcomes for the significant portion of patients treated non-operatively were poor.

Presentation \#93. The Posterior Use of BMP-2 in Cervical Deformity Surgery Does not Result in Increased Peri-Operative Complications: A Prospective Multicenter Study

Introduction: Although the use of recombinant human bone morphogenetic protein-2 (BMP-2) is contraindicated in anterior cervical procedures, retrospective series have shown that it can be safely using in posterior cervical procedures. However, the rate of complications following BMP use in the posterior cervical spine has not been studied in a prospective series of adult cervical deformity (ACD) patients.

Methods: This was a prospective cohort study seeking to enroll operative cervical deformity (CD) patients. The inclusion criteria were one or more of the following: cervical kyphosis $(\mathrm{CK})>10^{\circ}$, cervical scoliosis $(\mathrm{CS})>10^{\circ}, \mathrm{C} 2-7 \mathrm{SVA}>4 \mathrm{~cm}$ and / or chin-brow vertical angle $(\mathrm{CBVA})>25^{\circ}$. Demographic, operative and radiographic variables were recorded. Intermediate (3 month) and 6 month follow up was obtained. Patients were divided into two groups: those who received BMP-2 (BMP) and those who received no BMP-2 (NoBMP). Patient demographic data, operative details, and radiographic parameters were compared. Patients with neurologic complications were identified. Statistical analysis was performed with an independent t-test for continuous variables or Chi-square test for categorical variables. In the
BMP group, the relationship between BMP use (total, dose per level) and complications was also evaluated using linear regression.

Results: A total of 100 patients were included. Average age was 61.5 yrs with $40.8 \%$ males and $59.2 \%$ females. Average follow up was 7.6 months. There were 47 patients in the BMP group and 53 in the NoBMP group. An average of $13.6 \mathrm{mg}$ of BMP was used per person with $1.5 \mathrm{mg}$ per level. BMP was used posteriorly only. Compared to the NoBMP group, patients in the BMP group were older $(\mathrm{p}=0.03)$ and had longer prior fusions (6.0 vs. 2.5 levels, $\mathrm{p}<$ 0.01). There was no difference between the BMP and NoBMP groups with regards to: revision vs. primary surgery, Charlson Comorbidity Index (CCI), estimated blood loss (EBL), operation time, fusion levels, surgical approach, posterior osteotomy and anterior corpectomy. Radiographically, there was no difference between the two groups in maintenance of the radiographic parameters at 6-month follow up. There was no difference between groups with regards to complication incidence, total complications per person, major complications per person or any specific complication (e.g., neurologic complications, wound complications, etc.) Furthermore, a linear regression in the BMP group showed no predictive relationship between the total dose of BMP and incidence of total, minor or each specific complication $(\mathrm{p}>0.05)$. Also linear regression did not reveal predictive relationship between the total dose of BMP and fusion levels, the incidence of major or operative complications ( $\mathrm{r} 2=0.09,0.08,0.06)$ despite statistical significance.

Conclusion: The posterior use of BMP was not directly associated with an increased incidence of complications in this prospective cohort of operative Adult Cervical Deformity patients. BMP use was associated with older patients and with longer fusions.

Disclaimer: The Journal of Neurosurgery Publishing Group (JNSPG) acknowledges that the preceding abstracts and poster sessions are published verbatim as submitted and did not go through either the JNSPG's peer-review or editing process. 